Portland State University

PDXScholar

Fall 1-18-2019

\title{
Application of Improved Feature Selection Algorithm in SVM Based Market Trend Prediction Model
}

Qi Li

Portland State University

Follow this and additional works at: https://pdxscholar.library.pdx.edu/open_access_etds

Part of the Electrical and Computer Engineering Commons Let us know how access to this document benefits you.

\section{Recommended Citation}

$\mathrm{Li}, \mathrm{Qi}$, "Application of Improved Feature Selection Algorithm in SVM Based Market Trend Prediction Model" (2019). Dissertations and Theses. Paper 4730.

https://doi.org/10.15760/etd.6614

This Thesis is brought to you for free and open access. It has been accepted for inclusion in Dissertations and Theses by an authorized administrator of PDXScholar. Please contact us if we can make this document more accessible: pdxscholar@pdx.edu. 
Application of Improved Feature Selection Algorithm in

SVM Based Market Trend Prediction Model

by

Qi Li

A thesis submitted in partial fulfillment of the requirements for the degree of

\author{
Master of Science \\ in \\ Electrical and Computer Engineering
}

Thesis Committee:

Fu Li, Chair

James Morris

Xiaoyu Song

Portland State University

2018 
(C) 2018 Qi Li 
Abstract

In this study, a Prediction Accuracy Based Hill Climbing Feature Selection Algorithm (AHCFS) is created and compared with an Error Rate Based Sequential Feature Selection Algorithm (ERFS) which is an existing Matlab algorithm. The goal of the study is to create a new piece of an algorithm that has potential to outperform the existing Matlab sequential feature selection algorithm in predicting the movement of S\&P 500 (^GSPC) prices under certain circumstances. The two algorithms are tested based on historical data of ^ GSPC, and Support Vector Machine (SVM) is employed by both as the classifier. A prediction without feature selection algorithm implemented is carried out and used as a baseline for comparison between the two algorithms. The prediction horizon set in this study for both algorithms varies from one to 60 days. The study results show that AHCFS reaches higher prediction accuracy than ERFS in the majority of the cases. 


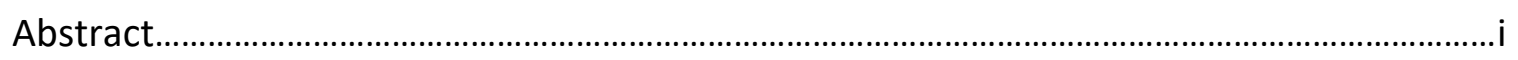

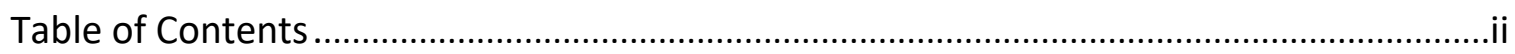

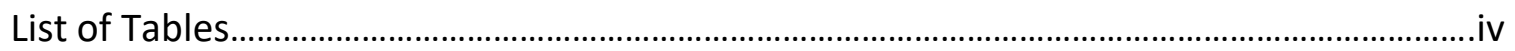

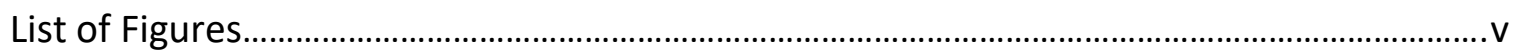

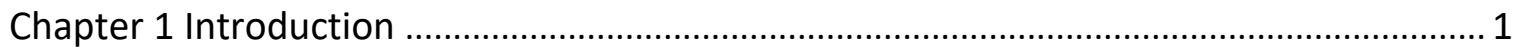

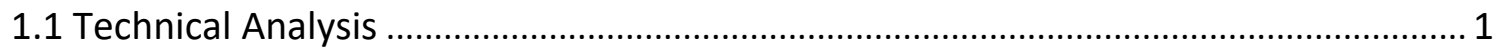

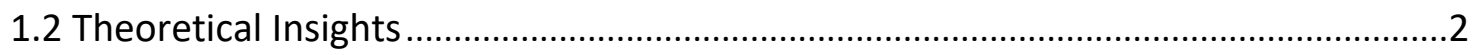

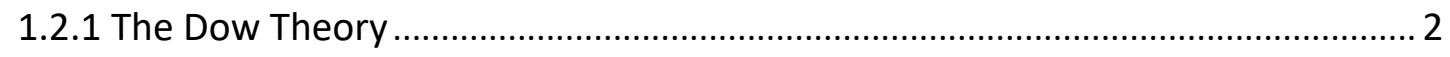

1.2.2 Efficient Market Hypothesis..............................................................................

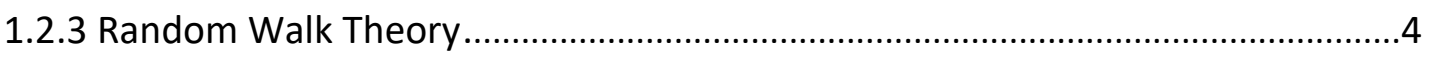

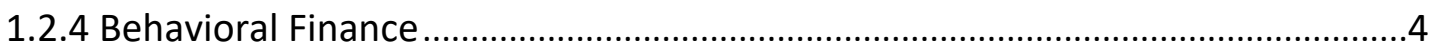

1.3 Combination of Technical Analysis and Artificial Intelligence ...................................5

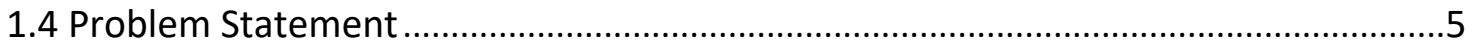

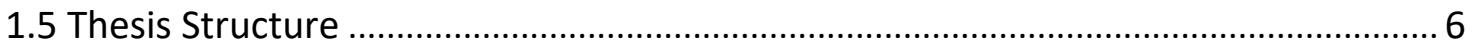

Chapter 2 Background Information and Literature Review .............................................. 7

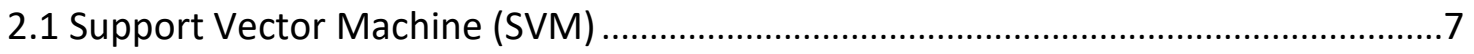

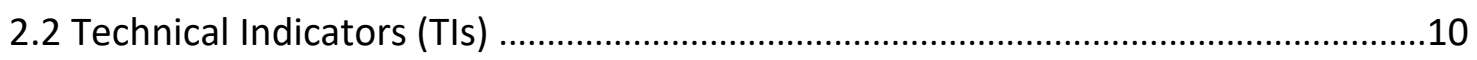

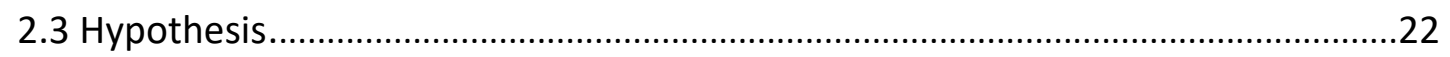

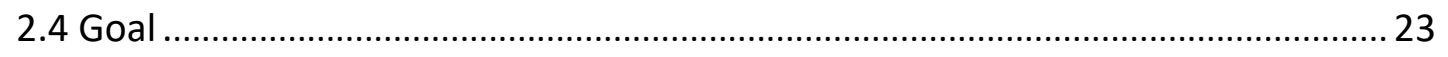

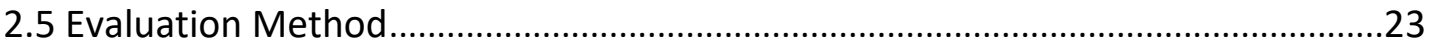

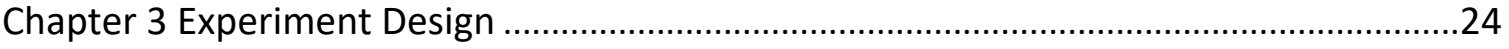

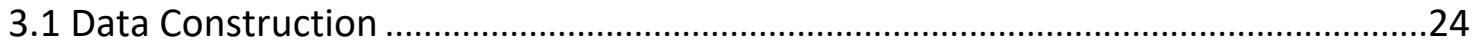

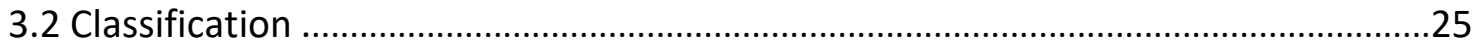

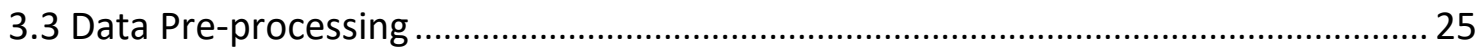

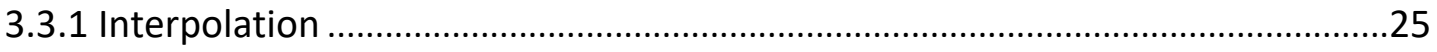

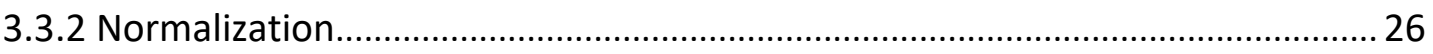




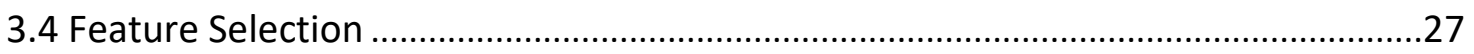

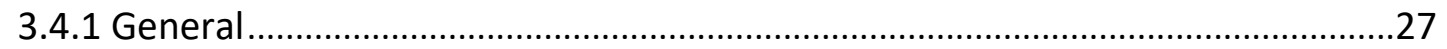

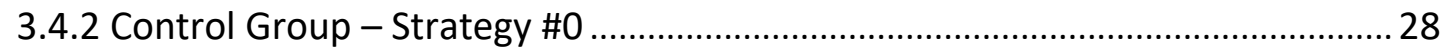

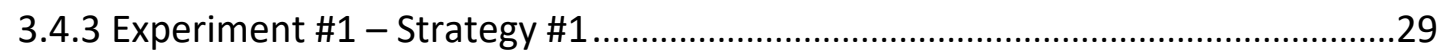

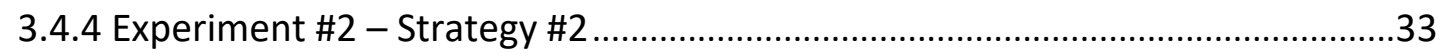

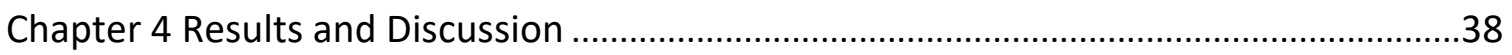

4.1 Effectiveness and stability of SVM based feature selection algorithm .....................38

4.2 Scenario 1: Experiment based on the most recent data (2007-2017) .....................39

4.3 Scenario 2: Experiment based on data with sharp changes (2000-2010) ................ 45

4.4 Scenario 3: Experiment based on older data (1992-2002) ........................................50

4.5 Scenario 4: Experiment based on much older data (1960-1970) ..............................55

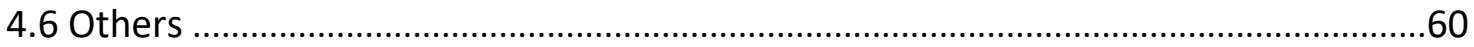

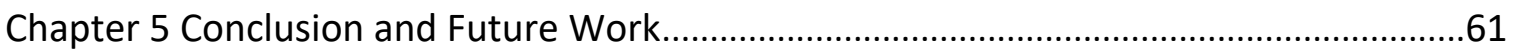

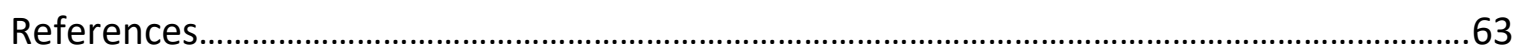

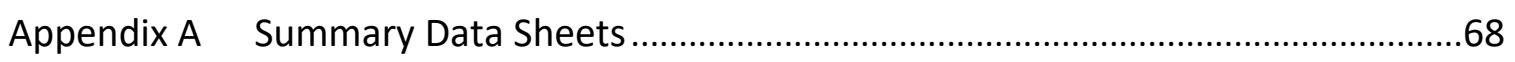

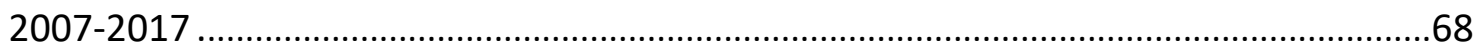

2000-2010

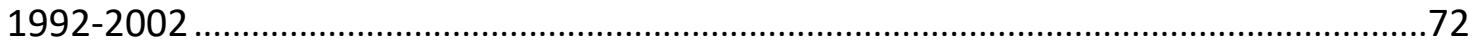

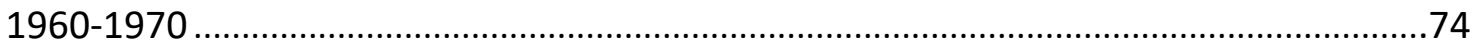

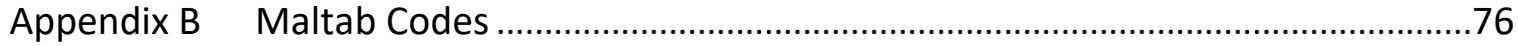

SVM based prediction with AHCFS as a feature selection method ................................76

SVM based prediction with ERFS as a feature selection method ...................................81

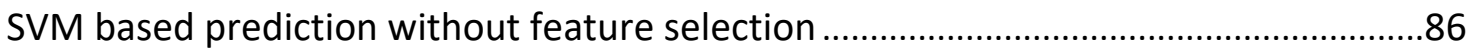

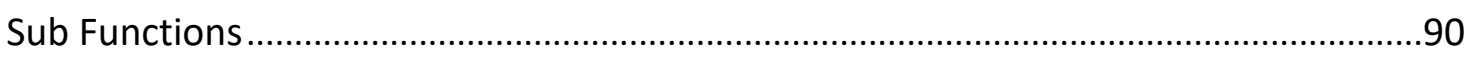

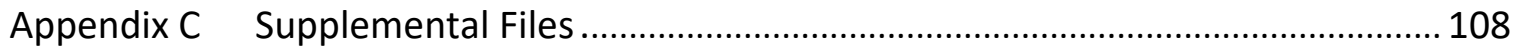




\section{List of Tables}

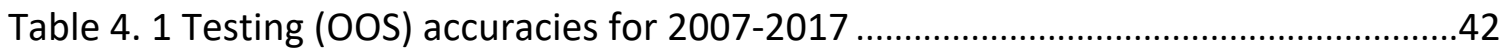

Table 4. 2 Improvement by ERFS vs. by AHCFS (baseline: no feature selection) ..............43

Table 4. 3 Testing (OOS) accuracies for 2000-2010 ….....................................................47

Table 4. 4 Improvement by ERFS vs. by AHCFS (baseline: no feature selection) ..............48

Table 4. 5 Testing (OOS) accuracies for 1992-2002 ….......................................................52

Table 4. 6 Improvement by ERFS vs. by AHCFS (baseline: no feature selection) ..............53

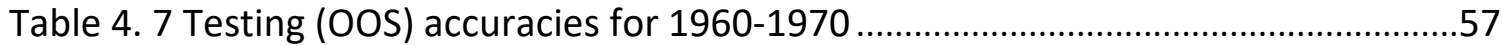

Table 4. 8 Improvement by ERFS vs. by AHCFS (baseline: no feature selection) ..............58 


\section{List of Figures}

Figure 3. 1 Flowchart of SVM classification model without feature selection (Strategy \#0) ........ 27

Figure 3. 2 Flowchart of SVM classification model with feature selection (Strategy \#1 \&

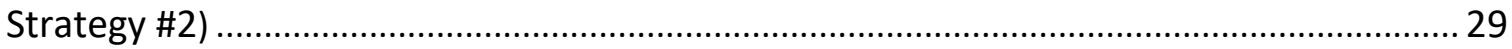

Figure 3. 3 Flowchart of feature selection (Part 1) …............................................................30

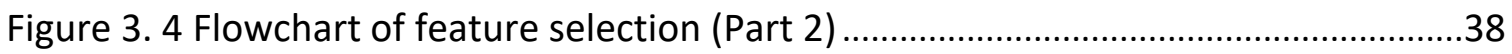

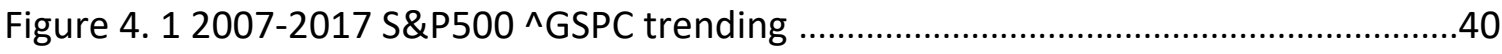

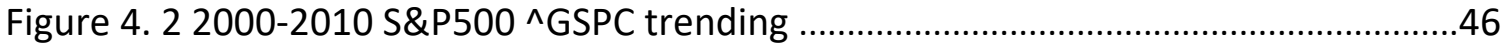

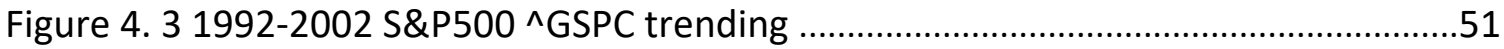

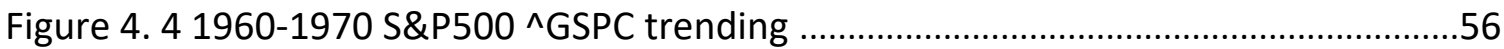


Chapter 1 Introduction

Financial markets are becoming more and more significant in the modern economic system [1]. Nowadays, the stock market is an essential component of the global economy. Each stock market plays a pivotal role in many fields, and the state of a nation's stock market somewhat represents its economic status. Due to the fundamental importance of stock markets, significant effort has been put into studies regarding market behaviors. One of the many major topics is predicting stock price.

\subsection{Technical Analysis}

Technical analysis seems to have first appeared in $18^{\text {th }}$ century Japan [2]. The first version, the Japanese version, of technical analysis was based on candle charts. It is currently one of the most popular tools and was first created and used by a wealthy merchant.

In recent years, computing power is becoming more powerful and cheaper. Thus it is more accessible. Meanwhile, artificial intelligence develops rapidly, such as machine learning. Many efforts have been put into the study of predicting stock prices and many theories have come into being [3]. Besides the traditional analysis method, fundamental analysis (FA), a new method, technical analysis (TA) is actively used in stock price forecasting [4]. The FA evaluates the intrinsic value of a company's stock price by delving into the company's financial statements [5]. The FA evaluation is a quantitative analysis based on the company's revenues, expenses, assets, liabilities, and all the other financial aspects. The analysis will yield a lot of measurements of the company's 
financial status as well as a comprehensive projection based on experience-adjusted risk parameters. These will then be used to determine the intrinsic value of a company's stock price. On the other hand, the TA, as a nontraditional method, is quite the opposite. The TA method uses a company's historical data regarding stock price, other related prices, and other non-price information to identify and summarize existing patterns and to suggest future movements instead of measuring intrinsic value.

More straightforward, the FA method predicts stock price by finding out and explaining what the underlying assets of the stock are and how the stock works; the TA method predicts the stock price by only tracking and learning the stock price and related information to find a pattern for future use. In sum, FA seeks to understand and explain the entire system to provide a prediction while TA lets the data take priority and speak for itself.

\subsection{Theoretical Insights}

\subsubsection{The Dow Theory}

Charles Dow, the father of modern TA in the West, provides the initial basis for the further development of TA, which is now called the Dow Theory [6], [7]. Summarized by his followers, the Dow Theory includes six tenets/principles.

1. The Averages Discount Everything. Every single factor, which is likely to have an influence on both demand and supply, is reflected in the market price. 
2. The Market Has Three Trends. A primary trend lasts for more than a year; a secondary trend lasts from 3 weeks to 3 months; minor trends last less than three weeks.

\section{Major Trends Have Three Phases}

In the first stage, Accumulation Phase, investors enter; in the second stage, the Public Participation Phase, prices rapidly rise, and economic news becomes favorable; in the third state, the Distribution Phase, economic conditions peak, and public participation increases.

\section{The Averages Must Confirm Each Other}

The Industrial Average and Rail Average (now it is the Dow Jones Transportation Average) must confirm each other.

\section{Volume Must Confirm the Trend}

The Dow recognizes volume as a secondary indicator, ranked second only to price. Volume should expand in the direction of the primary/major (price) trend.

\section{A Trend Is Assumed to Be Continuous until Definite Signal of Its Reversal} Trends exist.

It occurs regardless of "market noise." Prices continue going in the same direction despite the short period of opposite movement. It lasts for a while until a reversal signal occurs.

Based on these six principles, TA is generally understood as based on the following three principles: 
- Price Discounts Everything

- Prices Move in Trends

- History Repeats Itself

\subsubsection{Efficient Market Hypothesis}

The Efficient Market Hypothesis (EMH) states that: "at any given time, security prices fully reflect all available information" [8]. EMH indicates that TA will not be useful. If the current price fully reflects all the information and states that previous prices cannot be used to predict future prices, EMH implies that no investment strategy can outperform the market.

\subsubsection{Random Walk Theory}

This theory states that stock market prices evolve according to a random walk and thus cannot be predicted [9]. It is consistent with $\mathrm{EMH}$ and contradicts the application of TA.

\subsubsection{Behavioral Finance}

$\mathrm{EMH}$ and random walk theories both ignore market realities by assuming that all participants are entirely rational. Behavioral finance studies investor's market behavior that derives from the psychological principles of decision making to explain why people buy and/or sell stocks [10]. A few of the behavioral biases discussed in this chapter might contribute to such trends and patterns. Further, TA based on historical data is able to discover trends and patterns to predict the future. 
With the support of behavioral finance, a new theory - Adaptive Market Hypothesis (AMH) - started to reconcile economic theories based on EMH with behavioral finance [11].

\subsection{Combination of Technical Analysis and Artificial Intelligence}

With the development of computational power in recent years, it is found that the application of artificial intelligence in TA can be very powerful and has excellent potential to bring changes on how to predict the market. A multitude of machine learning techniques is applied to TA to attempt to improve market prediction accuracy [12], [13], [14].

Simply put, TA uses technical indicators which are derived from stock prices, including open, close, high, and low prices, and volume as input to attempt to determine future trends and decide when to buy or sell stocks. Beyond this, there are many studies combining TA and artificial intelligence (AI), from which a much better prediction model could be achieved. For example, an Artificial Neural Network (ANN) is used for stock prediction [15], [16]. At present, Support Vector Machines (SVM), which works similarly to ANN, is used extensively in this field [17], [18].

\subsection{Problem Statement}

SVM works as a prediction model with a multitude of adjustable parameters. Technical indicators (TIs) are used as input features to fine-tune those parameters [19]. Then, the trained SVM based prediction model is used to predict future movement in stock prices. That leads to another question: how many and what kinds of indicators/information are 
best for SVM training? Common sense would suggest that the more information, the better. However, further studies indicate that increasing the number of SVM features will reduce performance. One important reason for this is the overfitting problem. This is the problem of feature selection [20].

Many algorithms have been developed to solve the problem of choosing the best features for SVM [21], among which the sequential feature selection function (a Matlab function "sequentialfs") is a good one [22]. This function is an error rate, filter-based, sequential feature selection algorithm (we call it ERFS in later discussion). Besides this function, another improved sequential feature selection method is developed and tested in this study, which promotes prediction accuracy. The improved method is a prediction accuracy based hill climbing feature selection algorithm or AHCFS.

\subsection{Thesis Structure}

Chapter 1 introduced the development of market prediction, stated the problem that will be covered in this thesis, and described the thesis structure. Chapter 2 provided the background information on previously-related works and clarified the thesis' hypothesis, goal, and evaluation method. Chapter 3 explained the experiment design and methodology. Chapter 4 displayed the results of experiments. Chapter 5 discussed and summarized the results along with all research aims. Chapter 6 concluded this research and suggested future applications and continuation of this research. 
Chapter 2 Background Information and Literature Review

Many studies have combined SVM and TA for market trend prediction/stock trading. Some begin by studying the features selecting problem to improve the SVM training process. The followings are the brief introduction to SVM, TIs, and ERFS algorithm which we used as a comparison of the AHCFS algorithm.

\subsection{Support Vector Machine (SVM)}

Support Vector Machine (SVM) [23], a supervised machine learning model [24], is one of the most popular machine learning algorithms. It is often used as a classifier in data classification, which is a common task of machine learning. The concept and the very first SVM algorithm were created by Vladimir N. Vapnik and Alexey Ya. Chervonenkis. Later, SVM was first introduced by Boser, Guyon, and Vapnik at the 1992 COLT conference [25]. Theirs SVM was developed from Statistical Learning Theory by Vapnik [26].

The basic idea of SVM is to create hyperplane to separate different classes of the data points. Let's only talk about two classes problem here. The hyperplane has one less dimension of the target space. For instance, if our two classes of data are distributed in 3-dimension space, the hyperplane the SVM created to separate the two classes is a 2dimension plane, a normal plane in the real world. If our data is in 2-dimension space, the hyperplane the SVM gives to separate the classes is a line. The following is the simplest example to demonstrate how SVM work. 


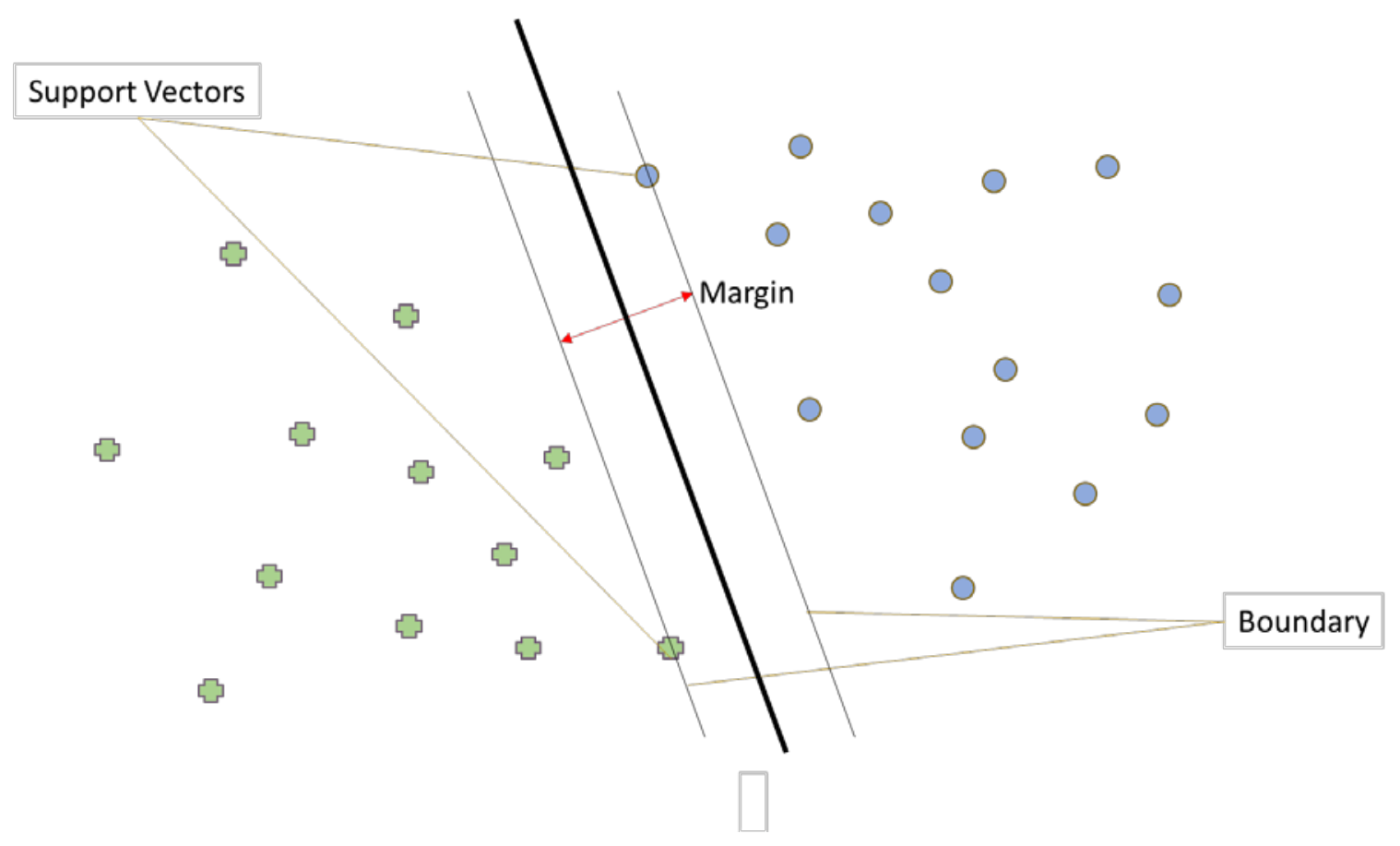

Figure 2. 1 Sample of how SVM works in 2-dimension case (not optimized)

In Figure 2.1, there are two classes of data, green cross group, and blue circle group, and SVM finds a line to separate the two groups of data reasonably. As shown in the figure, the thick black line is the separation line, and the two lighter black lines on both sides of the separation line are boundaries. Let the separation line moves parallelly towards both directions, the boundaries are determined as soon as the line reaches the very first data point or group of points. Moreover, the data points which define the boundaries are called support vector. Support vectors solely determine the boundaries. Also, the distance between the two boundaries, the red line in the figure, is the margin of this classifier. Apparently, the separation line in Figure 2.1 is not optimized, and its margin is not the largest. SVM tries to find a separation line which maximizes the margin under certain conditions. 


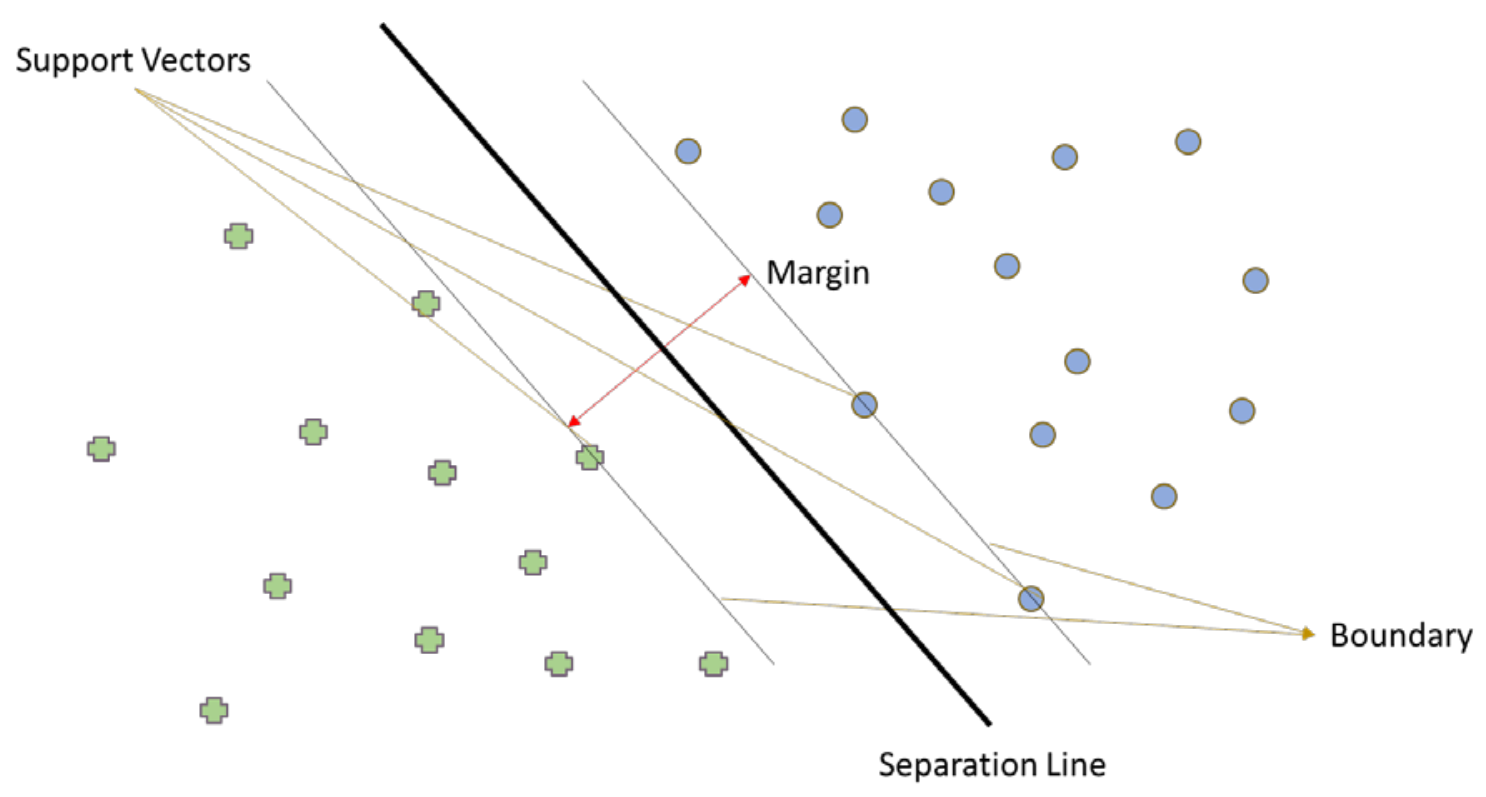

Figure 2. 2 Sample of how SVM works in 2-dimension case (optimized)

In Figure 2.2, the separation line with the maximized margin is given by SVM. Generally, for every classifier, the larger the margin is, the lower the classification error rate is.

SVM also uses kernel method [27]. In machine learning, SVM is not the only one which employs kernel method, but it must be the best-known one which employs a kernel method. The classification is a kind of analysis of relations between data points, so it usually does not need to calculate the coordinates explicitly. The high dimension data can be transformed using a user-specified kernel function without any explicit calculation of data point coordinates. The computing need of classification process is significantly lower after using the kernel function to transform. There are quite a few kernel functions that can be used in SVM; the commonly used kernel functions are the linear kernel, quadratic kernel, polynomial kernel, Gaussian Radial Basis Function (RBF) 
kernel, and Multilayer Perceptron (MLP) kernel. The RBF kernel is the most popular and most commonly used one, and it is used in this study as well.

The inputs of an SVM classification algorithm in Matlab are called features. In this study, all the inputs for SVM training or parameters tuning are constructed from technical indicators (Tis) [28].

\subsection{Technical Indicators (TIs)}

There are 24 TIs used in testing, and almost all of them come from the Technical Analysis Library (TA-lib). They are not equal to features, and features are made from them. There are 44 features made from TIs [29], [30].

2.2.1 Relative Strength Index (RSI)

$$
R S I=100-\frac{100}{1+R S}
$$

Where RS is the average upward price change divided by the average of downward price change over the same period.

RS compares the magnitude of recent gains and losses over a specified period, measuring the price movement and the changing speed of securities. It is used to identify the overbought/overvalued $(>70)$ or oversold/undervalued $(<30)$ status of certain assets.

In our Matlab code, the function is "TA_RSI," a 14-day period is used for RS. 


\subsubsection{Bollinger Bands}

Bollinger Bands are used to measure the volatility of a stock price and involves an upper and a lower band along with a simple moving average.

Bollinger Bands consist of:

- An N-period moving average

- An upper band at $\mathrm{K}$ times an $\mathrm{N}$-period standard deviation above the moving average

- A lower band at $\mathrm{K}$ times an $\mathrm{N}$-period standard deviation below the moving average

In our Matlab code, the function is "TA_BBANDS" and a 9-day period is used. Two times the standard deviation is used to determine the upper and lower band.

\subsubsection{Stochastic Oscillator}

The Stochastic Oscillator compares closing price of a stock to the range of its price over a specified period. This indicator includes two indicators: The Stochastic Fast (\%K) and the Stochastic Slow (\%D). Their formulas are:

$$
\begin{gathered}
\% K=100 \frac{C-L_{N}}{H_{N}-L_{N}} \\
\% D=\text { M period } S M A \text { of } \% K
\end{gathered}
$$

Where $C$ is closing price, $L_{N}$ is the lowest trading price of 14 previous trading days. $H_{N}$ is the highest trading price of the 14 previous trading days. The SMA stands for Simple 
Moving Average, which is explained below.

In our Matlab code, the function is "TA_STOCHF" and we use $N=14$ and $M=3$.

\subsubsection{Simple Moving Average (SMA)}

A Simple Moving Average (SMA) is an arithmetic moving average. The SMA is calculated by adding up the total of closing prices of the security for a few periods and dividing this sum by a pre-set number. Typically, the period is a day.

$$
S M A=\text { sum of closing price for } N \text { trading days } / N
$$

In our Matlab code, the function is "TA_SMA" and there are two sets of parameters used: the 10-day SMA and the 21-day SMA.

\subsubsection{Exponential Moving Average (EMA)}

The Exponential Moving Average (EMA) is another moving average but is like a simple moving average. The difference between EMA and SMA is that EMA weighs more recent data more heavily than less recent data. Here is the formula:

$$
E M A_{\text {today }}=C_{\text {today }} * K_{N}+E M A_{\text {yesterday }} *\left(1-K_{N}\right)
$$

Where $C_{\text {today }}$ is today's closing price, $N$ is the length of $E M A$. For example, if it is 4-day EMA, the $\mathrm{N}$ is $4 . K_{N}=2 /(N+1), E M A_{y e s t e r d a y}$ is the previous EMA value, calculated using the same formula.

In our Matlab code, the function is "TA_EMA" and we use a 4-day EMA. 


\subsubsection{Triple Exponential Moving Average (TEMA)}

Triple Exponential Moving Average (TEMA) is another moving average. It is a composite of a single exponential moving average, a double exponential moving average, and a triple exponential moving average. Here is the equation:

$$
T E M A=3 * E M A-3 * E M A(E M A)+E M A(E M A(E M A))
$$

Compared to EMA, TEMA smooths price fluctuations and filters out volatility, making it easier to identify trends with less lag time.

\subsubsection{Kaufman Adaptive Moving Average (KAMA)}

A moving average designed to account for market noise or volatility. It will closely follow prices when the price swings are relatively small, and the noise is low. KAMA will also adjust itself when the price swings, widens and follows price more loosely to keep it smooth. Here is the equation:

$$
\text { Current } K A M A=\text { Prior } K A M A+S C *(\text { Price }- \text { Prior } K A M A)
$$

$\mathrm{SC}$ is the Smoothing Constant which is calculated based on Efficiency Ratio (ER). ER is basically when the price change is adjusted for the daily volatility. Here are the equations:

$$
E R=\frac{A B S(\text { close price }- \text { close price }(10 \text { period ago }))}{\text { volatility }}
$$

Volatility is the sum of the absolute value of the last ten close price changes

$$
S C=(E R *(\text { fastest } S C-\text { slowest } S C)+\text { Slowest } S C)^{2}
$$


Here we use $\operatorname{KAMA}(10,2,30) .10$ is the number of periods for the $E R, 2$ is the number of periods for the fastest EMA constant (fastest SC), 30 is the number of periods for the slowest EMA constant (slowest SC).

2.2.8 Lowest Value \& Highest Value over a Specified Period (Min \& Max)

Those two technical indicators are merely the minimum and maximum value appearing over a certain period.

In our Matlab code, the function is "TA_MIN" and "TA_MAX." The period we used is a 5day period.

\section{*2.2.8 introduces two Tls.}

\subsubsection{Connors RSI (CRSI)}

Connors RSI has three major components: RSI, Updown Length, and ROC. RSI and ROC are introduced separately in this Chapter. Updown Length is the number of consecutive days that a security price has either closed up (higher than previous day) or closed down (lower than previous days). We usually use closing price as default. Closing up is a positive number, and closing down is a negative number.

CRSI has three variables, and here is the equation example for $\operatorname{CRSI}(3,2,100)$ :

$$
\operatorname{CRSI}(3,2,100)=\frac{R S I(3)+R S I(\text { Updown Length }, 2)+R O C(100)}{3}
$$


3 is the number of periods for RSI, 2 is the number of periods for Up-Down Length, 100 is the number of periods for ROC.

\subsubsection{Money Flow Index (MFI)}

The Money Flow Index measures the inflow and outflow of money into certain securities over a period. It uses a stock's price and volume to measure trading pressure. Here are the steps/items used to calculate MFI:

$$
\begin{gathered}
\text { Typical price }=(\text { high price }+ \text { low price }+ \text { closing price }) / 3 \\
\text { Raw money flow }=\text { typical price } * \text { volume }
\end{gathered}
$$

Money flow ratio $=(14-$ day Positive Money Flow $) /(14-$ day Negative Money Flow $)$

$$
M F I=100-\frac{100}{1-\text { Money flow ratio }}
$$

In our Matlab code, the function is "TA_MFI," and the function requires high, low, closing prices and volumes. No other parameter (number) is needed as input.

\subsubsection{Balance of Power (BOP)}

The Balance of Power (BOP) is designed to measure the strength of buyers versus sellers by assessing the ability of each to push the price to an extreme level.

$$
B O P=(\text { closing price }- \text { opening price }) /(\text { high price }- \text { low price })
$$


No other parameter/number is needed besides close, open, high, and low prices.

In our Matlab code, the function is "TA_BOP."

\subsubsection{Williams \%R (WPR)}

Williams \%R is also referred to as the Williams Percent Range (WPR). It also measures overbought and oversold levels. The equation is:

$$
\% R=\frac{(\text { high price }- \text { closing price })}{\text { high price }- \text { low price }} *-100
$$

In our Matlab code, the function is "TA_WILLR." No other parameter/number is needed besides high, low, and closing prices.

\subsubsection{Ultimate Oscillator (ULT)}

Ultimate Oscillator is a range-bound indicator. It uses the weighted average of three different periods to reduce volatility and false transaction signals. Before calculating ULT, we need to define several items:

$$
\begin{gathered}
\text { true low }=\min (\text { low price }, \text { previous closing price }) \\
\text { true high }=\max (\text { high price, previous closing price }) \\
\text { buying pressure }(b p)=\text { close }- \text { true low } \\
\text { true range }(\text { tr })=\text { true high }- \text { true low } \\
\qquad a v g_{7}=\frac{b p_{1}+b p_{2}+\cdots+b p_{7}}{t r_{1}+t r_{2}+\cdots+t r_{7}}
\end{gathered}
$$


Where $a v g_{7}$ is the sum of buying pressure over the most recent seven days divided by the sum of true range over those seven days. The same calculation applies to $\operatorname{avg}_{14}$ and $a v g$, and the ULT is:

$$
U L T=100 * \frac{4 * a v g_{7}+2 * a v g_{14}+a v g_{28}}{4+2+1}
$$

In our Matlab code, the function is "TA_ULTOSC." The period for the three averages in ULT calculation is adjustable. However, our setting for the three averages is the same as the example: 7,14 , and 28.

\subsubsection{Rate of Change (ROC)}

The Rate of Change (ROC) is the speed at which a variable will change over time. Here, ROC is used to describe the percentage of change in the value of a stock over a period. The equation is:

$$
R O C=\left(\frac{\text { current value }}{N-\text { day previous value }}-1\right) * 100
$$

In our Matlab code, the function is "TA_ROC." We use closing price as the value of a stock and let $N=5$.

\subsubsection{Average True Range (ATR) \& Normalized Average True Range (NATR)}

The Average True Range (ATR) is a measure of volatility. Before we calculate ATR, we define the true range as the following:

true range $(T R)=\max ($ high - low, abs $($ high - prev closing $)$, abs $(l o w-$ prev closing $)$ Where the high, low, prev close are high, low, and previous closing prices. 
The ATR is a moving average of the true ranges. The following is the ATR form of the exponential moving average:

$$
A T R_{\text {today }}=\frac{A T R_{\text {yesterday }} *(N-1)+\text { true range }_{\text {today }}}{N}
$$

Where $\mathrm{N}$ is the length of the moving average.

For the Normalized Average True Range (NATR), the formula is:

$$
N A T R=\frac{A T R_{N}}{\text { closing price }} * 100
$$

In our Matlab code, the functions are "TA_ATR" and TA_NATR." We use $N=14$ as suggested.

*2.2.15 introduces two Technical Indicators.

\subsubsection{Standard Deviation (SD)}

Standard Deviation (SD) is a fundamental measurement in descriptive statistics. SD measures the dispersion of a set of data from its mean. In investment, SD measures the volatility of the investments.

In our Matlab code, the function is "TA_STDDEV," and we calculate SD based on closing price and set the number of variable equals to 7 . 


\subsubsection{On-Balance Volume (OBV)}

On-Balance Volume is a momentum indicator that uses volume flow to predict changes in stock price. It is believed by the creator of the indicator that sharp increases in volume without a significant change in stock price will eventually lead to a jump forward in the price and vice versa.

The calculation of OBV is a running total of positive and negative trading volume for a stock. If today's closing price is above yesterday's closing price, today's trading volume is

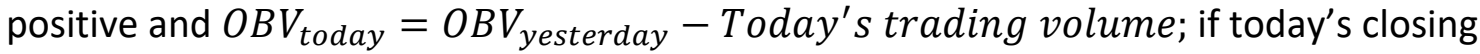
price is below yesterday's closing price, today's trading volume is negative and $O B V_{\text {today }}=O B V_{\text {yesterday }}-$ Today's trading volume.

In our Matlab code, the function is "TA_OBV" and there is no other parameter/number needed besides closing price and volume.

\subsubsection{Percentage Price Oscillator (PPO)}

Percentage Price Oscillator (PPO) is a momentum indicator that shows the relationship between two moving averages. Commonly, exponential moving averages are used in PPO. Here is the equation for PPO in terms of EMAs as an example:

$$
P P O=\frac{E M A_{N}-E M A_{M}}{E M A_{M}}
$$

Where $\mathrm{N}$ is a smaller number compared with $\mathrm{M}, E M A_{N}$ is a faster, short-term EMA and $E M A_{M}$ is a slower, long-term EMA. Usually, $\mathrm{N}$ is 9 and $\mathrm{M}$ is 26. 
In this Matlab code, the function is "TA_PPO" and we use $N=9$ and $M=26$. Besides $\mathrm{N} \& \mathrm{M}$, there is another parameter which is needed to set: the type of moving average. We use two which stands for exponential moving average form.

\subsubsection{Median Price}

The median price is merely the mid-point of a trading range for a period. It is an arithmetic average. The formula is:

$$
\text { Median Price }=\frac{\text { high price }+ \text { low price }}{2}
$$

In our Matlab code, the function is "TA_MEDPRICE" and there is no other parameter/number needed besides high and low price.

\subsubsection{Average Directional Index (ADX)}

The Average Index is used to qualify trend strength. It is a combination of two indicators: the Positive Directional Indicator (+DI) and the Negative Directional Indicator (-DI). To calculate +DI or -DI, we need to calculate the directional movement first (+DM or -DM):

$$
\begin{aligned}
& \text { UpMove = today's high }- \text { yesterday's high } \\
& \text { DownMove = yesterday's low - today's low }
\end{aligned}
$$

if UpMove $>$ DownMove and UpMove $>0$, then $+D M=$ UpMove, else $+D M=0$

$$
\begin{aligned}
\text { if DownMove } & >\text { UpMove and DownMove }>0, \text { then }-D M \\
& =\text { DownMove, else }-D M=0
\end{aligned}
$$


After +DM and -DM are calculated, + DI and $-\mathrm{DI}$ are:

$$
\begin{gathered}
+D I=100 * \frac{S M A_{N}(+D M)}{A T R} \\
-D I=100 * \frac{\left(S M A_{N}(-D M)\right)}{A T R}
\end{gathered}
$$

Then the ADX is:

$$
A D X=100 * S M A_{N}\left(\operatorname{abs}\left(\frac{+D I-(-D I)}{+D I+(-D I)}\right)\right.
$$

In our Matlab code, the function is "TA_ADX," and we use $N=14$.

\subsubsection{Chande Momentum Oscillator (CMO)}

Like RSI, the indicator is also used to measure the oversold $(+50)$ or overbought $(-50)$ status of certain securities. To get the number, first, we calculate the difference between the total of recent gains and the total of recent losses over a period. Then, we divide the difference by the total price movement over the same period. Let's define the total of gain, loss, and price movement:

$$
\begin{aligned}
& \text { if } \text { closing }_{\text {today }}-\text { closing }_{\text {yesterday }} \\
& >0 \text {, then closing } \text { today }_{-}-\text {closing }_{\text {yesterday }} \text { is gain. } \\
& \text { if } \text { closing }_{\text {today }}-\text { closing }_{\text {yesterday }} \\
& \left.<0 \text {, then abs (closing } \text { today }_{-}-\text {closing }_{\text {yesterday }}\right) \text { is loss. } \\
& \text { total of gains }=\operatorname{sum}_{N}(\text { gain }), \text { total of losses }=\operatorname{sum}_{N}(\text { loss }) \\
& \text { price movement }=\text { total of gains }+ \text { total of losses }
\end{aligned}
$$




$$
C M O=100 * \frac{\operatorname{sum}_{N}(\text { gain })-\operatorname{sum}_{N}(\text { loss })}{\operatorname{sum}_{N}(\text { gain })+\operatorname{sum}_{N}(\text { loss })}
$$

Where $\mathrm{N}$ is the period, for example, a 10-day period.

In our Matlab code, the function is "TA_CMO" and we use $N=10$.

\subsubsection{Commodity Channel Index (CCI)}

The Commodity Channel Index (CCl) is an oscillator and is used to measure whether a stock is oversold/overbought. It attains value by quantifying the relationship between the stock's typical price $\left(P_{t}\right)$, the $\mathrm{N}$ simple moving average of the stock's typical price $\left(S M A_{N}\left(P_{t}\right)\right)$, and the $\mathrm{N}$ points mean absolute deviation from typical $\operatorname{price}\left(\sigma_{N}\left(P_{t}\right)\right)$. Here is the formula:

$$
C C I=\frac{P_{t}-S M A_{N}\left(P_{t}\right)}{0.015 * \sigma_{N}\left(P_{t}\right)}
$$

Where the typical price is $P_{t}=\frac{h i g h+\text { low }+ \text { closing }}{3}$, and the aim of scaling by $1 / 0.015$ is to produce a more readable number.

In our Matlab code, the function is "TA_CCl", and we use $N=20$.

\subsection{Hypothesis}

Although the financial market is complex, based on previous research, market trends are somewhat predictable. The TA method offers a unique way to discover the secret of future movement. A machine learning classifier such as SVM is a great tool that significantly improves market trend prediction. An SVM based TA for market trend prediction is a beautiful approach, and we believe it is possible to improve the 
predicting accuracy by performing features selection while preparing training data for an SVM based prediction model. Furthermore, improvement of the feature selection algorithm can promote the prediction accuracy of the model again.

\subsection{Goal}

The goals of the work include:

1. Study prediction accuracy improvement after applying Sequential Feature Selection function to an SVM based model.

We will use a fixed, initial combination of technical indicators as input to train SVM and test the model. Then we apply ERFS on the same initial combination of technical indicators, using the left indicators to train SVM and test the model. The differences between the two groups of results will be studied.

2. We will apply the AHCele instead of the ERFS and repeat the test. The new group of results is then studied and compared to the previous two result groups. The differences will then be interpreted.

\subsection{Evaluation Method}

The critical measurement of the prediction model is the prediction accuracy, and all results are finally evaluated by their prediction accuracy. The prediction model is trained and tested/simulated based on historical data using Matlab. The classifier used for predicting target is SVM. The prediction accuracy is defined as the sum of correctly classified targets divided by the sum number of targets. 
Chapter 3 Experiment Design

The whole experiment is carried out in Matlab based on its easily accessible and powerful simulator. The market prediction is basically an application of the SVM classifier. The data used to train the SVM classifier include features made from technical indicators based on historical data from the S\&P500 (^GSPC). Later we will use the SP500 instead of the S\&P500 (^GSPC). The trained SVM classifier is also tested on historical data from the SP500.

\subsection{Data Construction}

The original data is historical S\&P 500 index prices (^GSPC) acquired from Yahoo! Finance. The available data spans from 1950 to 2017. This data is composed of daily data points. Each data point consists of high, low, close, and open prices and volume of a particular trading day. All the 24 technical indicators introduced in Chapter 2 are calculated based on the market high, low, close, and open prices and volume and are further made into 44 features. The features are the inputs for SVM training. Although other indicator types are prepared, such as microeconomic indicators (other stocks' momentum and acceleration) and microeconomic indicators, they were not used in our experiments.

Among the 67 years of data, we take a 10-year window divided into two parts -the first part is for the training aim, called In-Sample data; the second part is for the testing aim, called Out-Of-Sample data. The length ratio of In-Sample data to the length of Out-Of- 
Sample data is 7:3. The In-Sample is used for features selecting, and the data after selection are for SVM training inputs. The Out-Of-Sample part is used for testing the trained SVM classifier and returns a prediction accuracy used to evaluate the trained model. There are approximately 252 trading days in a year, which makes about 1800 points in the In-Sample data and 800 points in the Out-Of-Sample data when daily data is used.

In the following experiments, four pieces of data are used: 2007-2017, 2000-2010, 19922001, and 1960-1970.

\subsection{Classification}

The prediction model is designed to indicate the market trend; it is the movement of the S\&P 500 index price in this case. All the data used as inputs are sharing the same unit: the U.S. dollar. Based on the above information, the classification criterion is to check the difference between the present index closing price and the previous index closing price. If the difference is non-negative (including 0 ), which means the index closing price does not fall, the classification result and record is a 1; if the difference is negative, which means the index closing prices fall, the classification result and record is a 0.

\subsection{Data Pre-processing}

\subsubsection{Interpolation}

It is a necessary process before the data enters into the SVM. Due to missing values in stock prices (or/and character of feature formulas), some "not a number" (NaN) values appear, which can break SVM training rules and ruin the training process. Those NaN 
values are converted into zeros in all following experiments to make the SVM work normally. There is another way to interpolate these kinds of numbers using linear regression or the average of several neighboring numbers to smooth out the holes. Typically, this method eliminates the holes inside the training data more smoothly than inserting zeros. However, when there is a string of NaN values, especially when starting from the very beginning of the array, this method will not work well.

\subsubsection{Normalization}

After features are calculated, the magnitude order of features could be very different. Some range from 0 to 1 or -1 to 1 , while others range from 1 to 10,000 . Their scales can be incredibly different and will likely poorly affect the performance of the classifier, which is trained via this data. It will significantly lengthen the running time of classifier training. One reason is the mixed use of prices and volumes. Prices are recorded in the hundreds or thousands of levels, but volumes are recorded in tens of millions. The other reason is that all kinds of technical indicators are employed, some are designed to determine percentages, and some are designed to determine large numbers. It is essential to implement normalization to make the data organized and efficient. The Matlab function "zscore", which performs better than another two choices "normc" and "normalize", according to a summing-up in a similar work [31], is used in here to normalize all the data.

With the character of each technical indicator/feature formula known, there is another to avoid normalization while keeping data efficient. We can rescale each 
indicator/feature to let their order of magnitude become more consistent before pouring them into the SVM. This idea is tested and discussed separately at the end.

\subsection{Feature Selection}

\subsubsection{General}

Feature selection (FS) is a great way to screen data and improve the accuracy of a prediction model [32]. Feature selection is a critical part of the following experiments.

The experiment designed a comparison among one control group and two experimental groups. All three codes, including raw data processing, features calculating, and SVM training, testing, and generating output, are the same except for feature selection parts. For feature selection part, each group used different strategies.

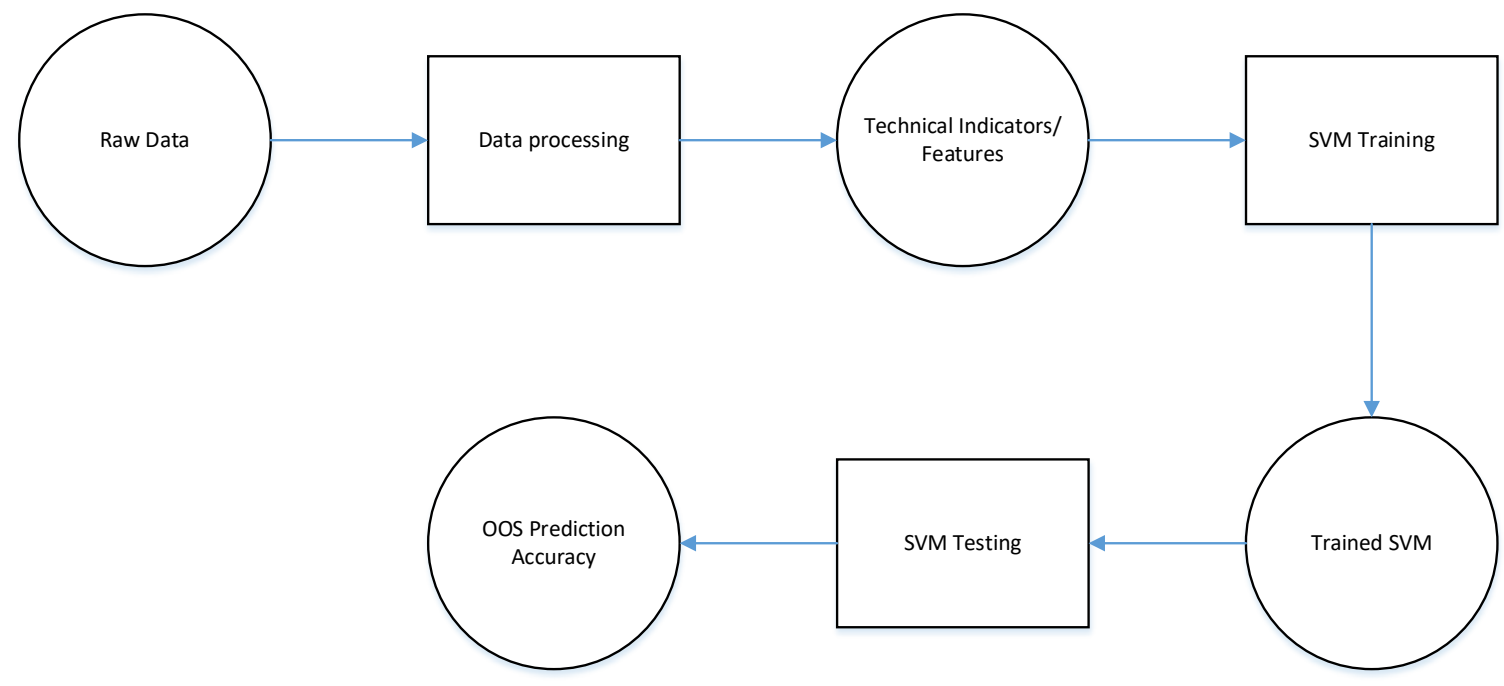

Figure 3. 1 Flowchart of SVM classification model without feature selection (Strategy \#0) 


\subsubsection{Control Group - Strategy \#0}

The flowchart of the code used in the control group is given above. We call this Strategy \#0 (Without FS). Basically, the code in Strategy \#0 includes no feature selection algorithm. It just merely uses all initial features as input for SVM training.

First, the code acquires raw data from a prepared Excel file created directly by Yahoo! Finance. The Excel document (.csv format) is the historical S\&P $500 \wedge$ GSPC daily data beginning in 1950, including open price, high price, low price, closing price, and daily trading volume. Second, all the raw data goes into processing algorithm. All the data will be extracted and separately stored. Date format will be changed, and step size (prediction horizon) will be set. All 24 technical indicators will be calculated, and further calculation is conducted to get all 44 initial features' values from the technical indicators. Data piece boundaries will be set, and training and supervising groups will be created. Third, after data processing, the prepared features input will be used in training the SVM, and the trained SVM classifier will be employed as our prediction model. Last, the testing group data created in the above step will be used to get the Out-of-Sample prediction accuracies, which we will use to evaluate the effectiveness of the prediction model. 


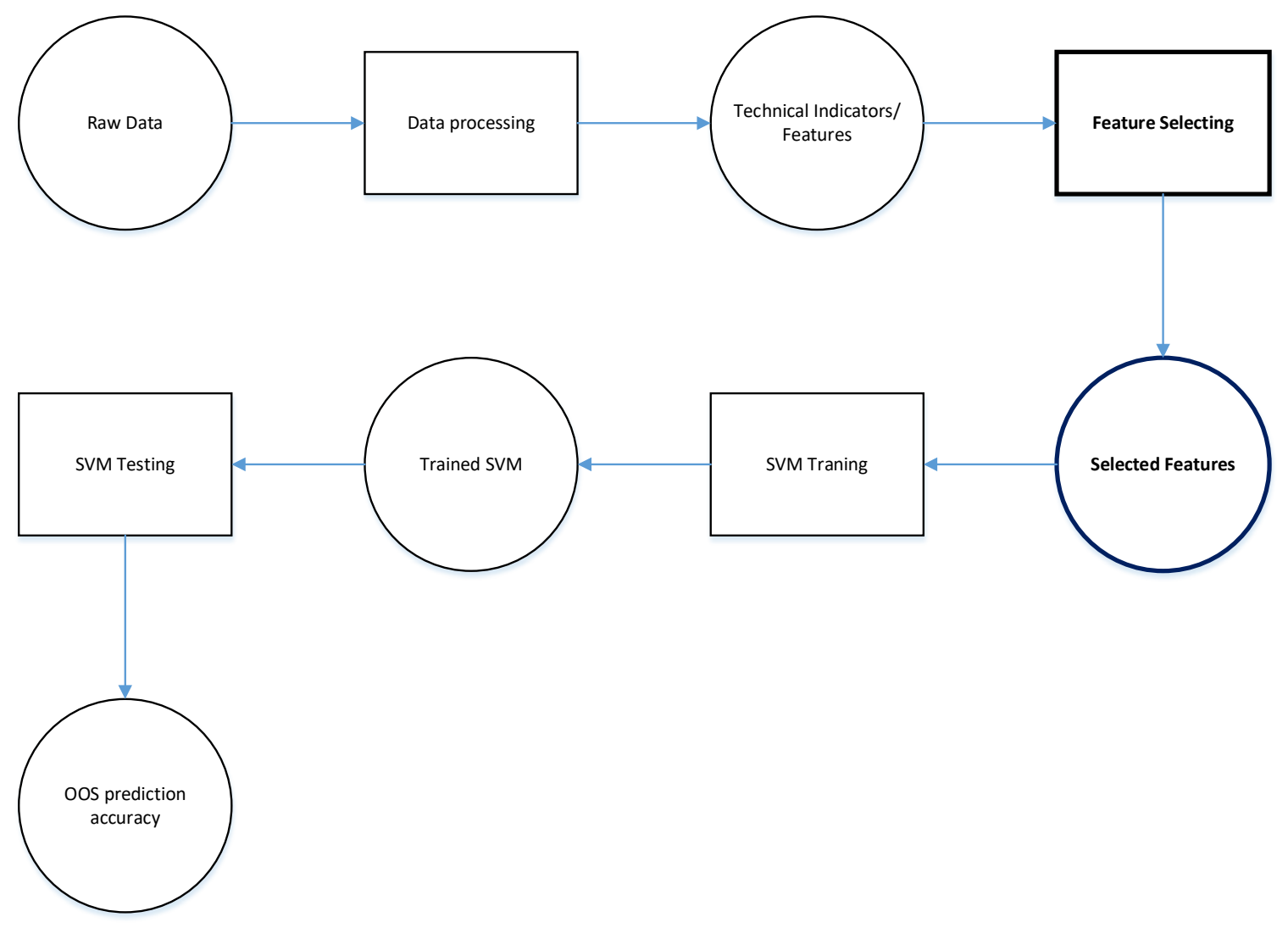

Figure 3. 2 Flowchart of SVM classification model with feature selection (Strategy \#1 \& Strategy \#2)

Although the algorithms are different from each, both can fit into the same flowchart above.

\subsubsection{Experiment \#1 - Strategy \#1}

The code in the experiment \#1 group will employ an Error Rate Filter Sequential Feature Selection (ERFS) algorithm as its feature selection part. We call this Strategy \#1. It is a partial Matlab function that provides a variety of changeable parameters and settings plus a user given function that calculates criterion values to rank features. Compared to a wrapper method, it is more like a filter method. Thus, to provide the same basis in 
comparison, a piece of code is added to transform it into a wrapper method. Besides features selection, all parameters and settings are the same as in any of the experiments. The following is the flowchart of Strategy \#1 feature selection following the detailed introduction of Strategy \#1.

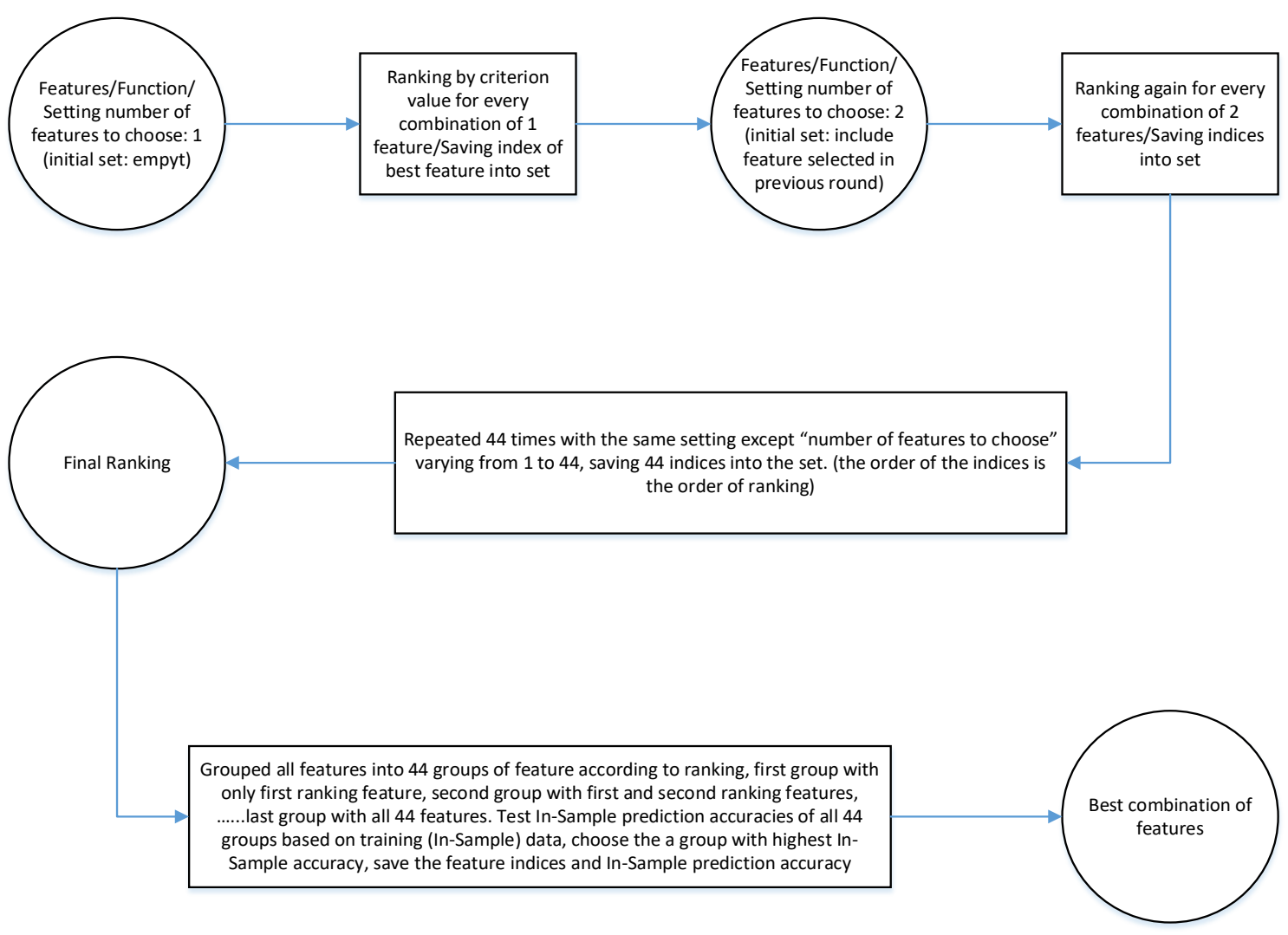

Figure 3. 3 Flowchart of feature selection (Part 1)

The inputs of the Strategy \#1 algorithm include all 44 features, the function given by use for calculating ranking criterion value, and the number of features - varying from 1 to 44 with the ranking algorithm repeated 44 times - to choose. It is a forward sequential features selection starting from an empty set of features and individually adding features. The function chosen by the user, in this case, is a function that calculates the 
error rate based on the SVM classification. The error rate is calculated based on InSample data using 10 -fold cross-validation. We will call this the "error rate function" in all following discussions. The ranking process will be applied multiple times round by round. In any given round, each possible combination that meets the conditions is evaluated by the error rate function, and an error rate based on In-Sample data will be returned. In that round, the one with lowest error rate will be chosen and the feature indices will be saved and will serve as the initial set for the next round. It will repeat 44 times before finishing all evaluations and rankings.

For example, in round 1 , with the features and function as input, the number of features to choose from is set 1 , and the initial evaluating starts with a starting set of 0 features. The algorithm then chooses feature 1 (feature one is not first ranking feature, but the feature with index 1 in the coding) each time, calculates the error rate after each selection, saves, initializes a starting set of features to empty, chooses feature 2 . It then repeats 44 times until every possible combination is evaluated, saves 44 error rates, choosing the one with the lowest error rate, saves the index as the first ranking feature, and adds it into a starting set of features for the next round. As round 1 finishes, round 2 starts. In round 2, with the same features and function as input, the number of features to choose is pre-set to be 2 , and the initial evaluating begins with a starting set of first ranking features. The algorithm then chooses feature 1 in left 43 features to combine with the first ranking feature, calculates the error rate, saves, and initializes the starting set of features (a set only includes first ranking features). The algorithm then selects, 
evaluates, saves again, and repeats 43 times until all possible combinations are evaluated, and all error rates are achieved. The combination with the lowest error rate is chosen, and the index with first and second ranking features is saved (the first ranking one is always the feature selected in round 1) and then added into the starting set of features for the next round. Round 2 ends and round 3 begins. This process repeats multiple times until round 44 , with only the number of features to choose from changing. There is only one possible combination - a combination of all 44 features. After the last round (round 44 in this case), all 44 features are ranked.

To compare with another wrapper method of feature selection, we need to figure out exactly which combination of features provides the highest prediction accuracy. With the ranking of features known, we need to decide how many features from top to bottom, if chosen, will yield the best prediction accuracy. Due to the nature of 10 -fold cross-validation, the error rate here is not a real prediction error rate. It is not proper to use the error rate derived from a 10 -fold cross validation as evaluation of prediction accuracy. The error rate is more like an evaluation of each feature itself and the feature selection method up to this point is more like a filter method than a wrapper method. For the aim of comparing the method with another wrapper method, another evaluation method is needed to be added to assess and find the combination, with the ranking known, that gives the highest In-Sample prediction accuracy. The following process is added to achieve the evaluation aim. 
Following the previous output and according to the ranking, all 44 features are allocated into 44 groups: group 1 includes the first ranking feature, group 2 includes the first and second ranking features, group 3 includes the first, second, and third-ranking features and so on. Group 44, of course, includes all features. All the groups are evaluated by a "hold-out" cross-validation and will return an In-Sample prediction accuracy. The group with the highest In-Sample accuracy is the best combination of features selected by feature selection Strategy \#1.

It is an existing feature selection method from a previous paper.

\subsubsection{Experiment \#2 - Strategy \#2}

The code in the experiment \#2 group will employ the AHCFS algorithm as its feature selection part. We will call this Strategy \#2. It consists of two processes. The first one is like the one in Strategy \#1, and we will call it Step \#1 in Strategy \#2. The second one is a conditional exhaustive search algorithm (Hill Climbing Scheme), and we will call it Step \#2 in Strategy \#2.

To introduce Step \#1 of Strategy \#2, please refer to figure 3.2 - the flowchart of Strategy \#1, which is also suitable for Step \#1. There are two main differences here:

1. In Strategy \#1, it uses an error rate to choose the best combination and further decides the ranking. In this way, it can only rank one feature each time and needs to repeat 44 rounds to rank all 44 features. In Strategy \#2, Step \#1, it uses prediction accuracy (this is the same as the error rate to some degree. Theoretically, prediction accuracy equals one minus the error rate of each 
feature itself to rank the feature. In this way, there is only one round of calculation needed to rank all features.

2. The validation methods used to calculate the error rate/prediction accuracy are different. In Strategy \#1, it uses a 10-fold cross validation to calculate the error rate. Due to the nature of 10 -fold cross-validation, the validation process is less like a prediction process. In Strategy \#2 Step \#1, it uses hold-out cross-validation, which exactly the prediction process uses. The validation process on In-Sample data is more consistent with the final testing process on Out-of-Sample data and helps to improve the effectiveness of feature selection.

Since it uses hold-out cross-validation, we need to break the In-Sample into "InSample" and "Out-of-Sample" parts and attend that the code does not know the "Out-of-Sample" part while training the model. We can name the pseudo-InSample and pseudo-Out-of-Sample or IS' and OOS' to distinguish from IS and Oos.

To introduce Step \#2 of Strategy \#2, please refer to Figure 5.4 below before we start. Step \#2 begins with the best combination of features and the highest IN-Sample accuracy from Step \#1. Process \#1 is an initial subtraction including indices of the best combination from Step \#1, and the IS accuracy of the combination will be the input. It subtracts one feature each time, calculates the In-Sample prediction accuracy, repeats as many times as possible until all possible subtractions are complete, and gets all the In-Sample accuracies, determining the highest one. The process then compares the highest IS accuracy generated from the initial subtraction and the highest IS accuracy 
from Step \#1. If the one from the initial subtraction is higher, the indices of combination and the IS accuracy of the combination will be saved. If not, then the original Step \#1 combination and IS accuracy is passed to the next process. However, whether the IS accuracy is improved or not, process \#1 ends and process \#2 begins.

Process \#2 is an addition cycle, and the indices of the best combination from process \#1 and its IS accuracy will be the inputs. It adds one feature each time, calculates the IS accuracy, repeats until all 44 possible additions are complete, and saves all the IS accuracies while determining the highest IS accuracy. It then compares the highest IS accuracy with the one passed from process \#1 (first subtraction) and decide. If the highest IS accuracy from this addition cycle is higher than the one passed from process $\# 1$, the indices of the new combination and its IS accuracy will be saved as new input and the addition cycle will be rerun from the beginning with the new input. Process \#2 then begins again. If it is not, the input of process \#2 will become the output of process \#2. When process \#2 ends, process \#3 begins.

Process \#3 is a subtraction cycle, similar to first subtraction, but a cycle. The inputs are indices of the best combination from process \#2, and its IS accuracy. It subtracts one feature each time, calculates the IS accuracy, repeats until all possible subtractions are complete, determines all IS accuracies and finds the highest one. Process \#3 then compares the highest IS accuracy after the subtraction cycle with the one passed from process \#2. If the highest IS accuracy from this subtraction cycle is higher than the one passed from process \#2, the indices of the new combination and IS accuracy of it will be 
saved as new input and the subtraction cycle will be rerun with an updated highest IS accuracy and the related combination as input. If it is not, the input of process \#3 will become the output as well. Process \#3 then ends and returns a finishing mark.

When process \#2 ends and process \#3 begins, the algorithm will count the number of times that process \#3 repeated and check the repeat times when a finishing mark appears. While checking, if the algorithm finds out that process \#3 is repeated once or more times, it will jump back to the beginning of process \#2, then process \#2 and \#3 run one more round with the final output of the previous round as input. If in a round, the repeat time of process \#3 equals zero while the finishing mark is reached, the entire feature selection is completed, and the final result is delivered. 


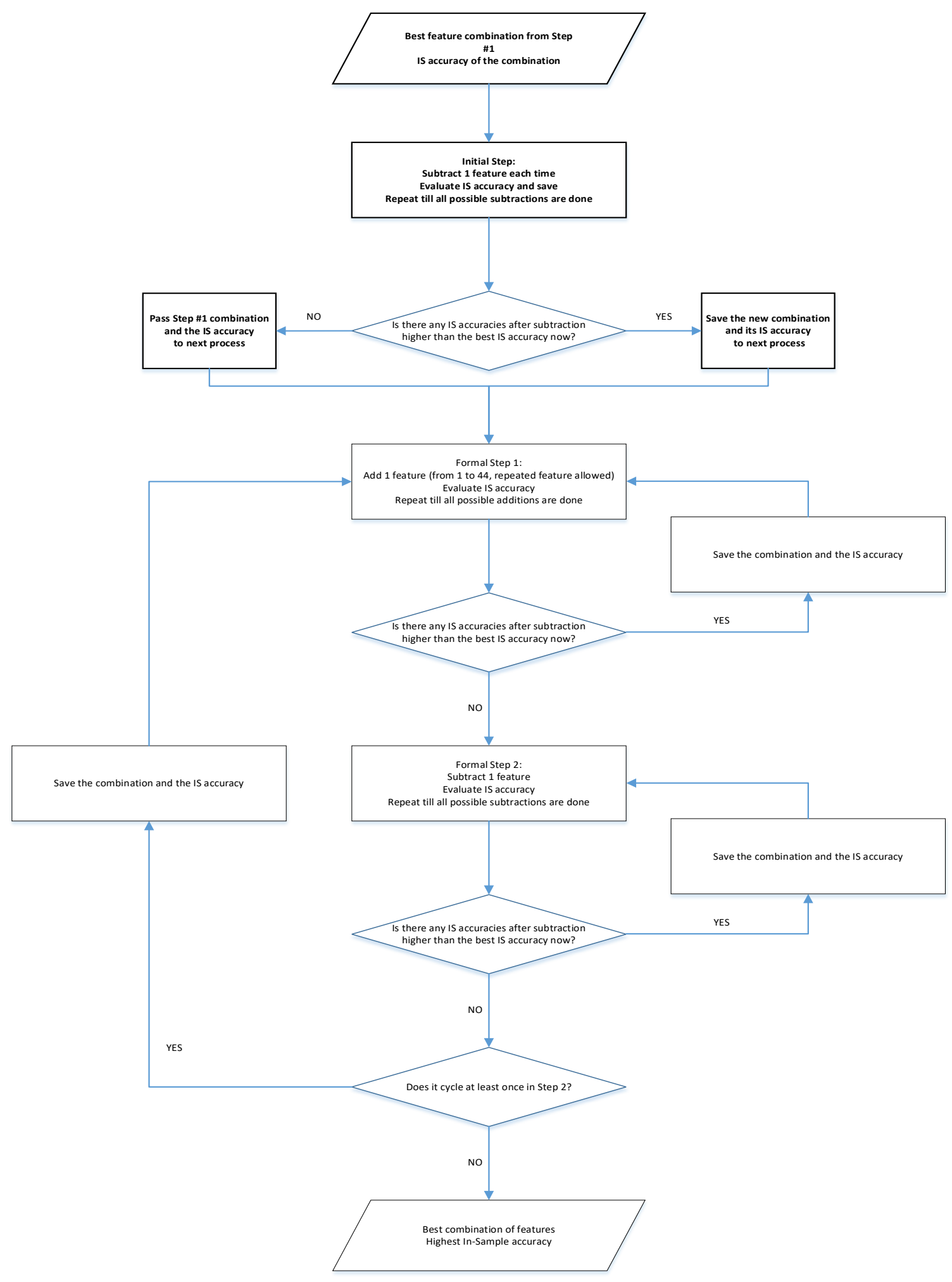

Figure 3. 4 Flowchart of feature selection (Part 2) 
Chapter 4 Results and Discussion

4.1 Effectiveness and stability of SVM based feature selection algorithm Each feature selection strategy/algorithm will finally generate the best combination of features after all, and each best combination is tested on testing (OOS) data - the unknown data - to evaluate the performance of each strategy. The three strategies/algorithms will be applied and tested on a different data period with the same supplementary parameters and settings. Two crucial metrics are measured to evaluate their performances:

- The effectiveness of feature selection strategy/algorithm - the absolute and relative percentage improvement on testing (OOS) prediction accuracy are the KPIs for evaluating the effectiveness

- The stability of feature selection strategy/algorithm in different economic environments - the fluctuation of feature selection effectiveness on different data period and the rate of sudden crush cases are the KPIs for evaluating stability. 
4.2 Scenario 1: Experiment based on the most recent data (2007-2017)

Parameters and settings are as follows:

1. Classification method - SVM
a. Kernel: Radial Basis Function
b. Normalization Method: zscore
c. Input features: 44 , all come from Technical Indicators

2. Training and testing data
a. Total length: 10 years
b. Training/Testing (IS: OOS) Ratio: 7:3
c. Data type: daily S\&P 500 ^ GSPC, prices, and volume
d. Date: IS $=2007-2014 ;$ OOS $=2014-2017$

3. Features selection:
a. Strategy \#0 vs. Strategy \#1 vs. Strategy \#2
b. Strategy \#2 Step \#1: IS': OOS': 2:5; IS' = $2007-2009$, OOS' $^{\prime}=2009-2014$

4. Step size (prediction horizon): from one day to 60 days (2 months)

The following are the trending of the S\&P500 ^ GSPC from 2007-2017 and the separation line of training (known) data and testing (unknown) data (Figure 4.1), and the results from all three algorithms (Strategy \#0, \#1, \#2) with varying step size (Table 4.1). Table 4.1 shows the testing (OOS) prediction accuracies of each strategy with different step size and the number of features selected in each case in terms of weekly averages and standard deviations. The original results worksheet is too big to be presented here. The 
step size varies from 1 to 60 ; in the weekly format, there are nine weekly averages.

From Figure 4.1, the trending in the training part included an economic downside at the beginning and turned back to rise without big relapses. The trend of the testing part is consistent with the trend of the second half of the training part. Normally, if the training part is somewhat like the testing part, the prediction result will be better than it is in general cases.

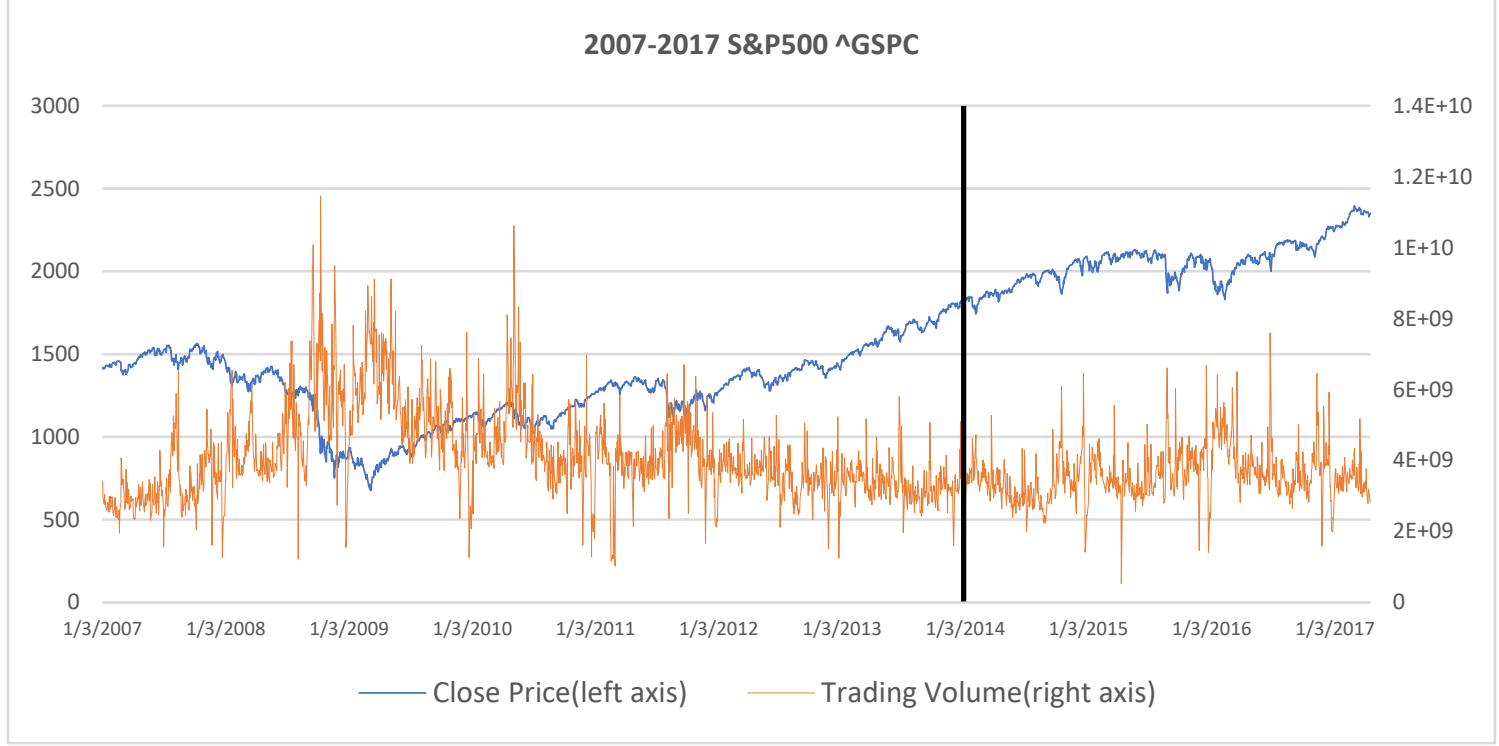

Figure 4. 1 2007-2017 S\&P500 ^GSPC trending

From Table 4.1, the weekly average shows that the testing accuracies of Strategy \#0 (no feature selection) increase when the step size increases from $55.55 \%$, when the step size is smaller than 7 ( 1 week), to $69.05 \%$, when the step size approaches 60 ( 2 months). Weekly standard deviations are less than $2 \%$ except for during the $1^{\text {st }}$ week, meaning there is less fluctuation in testing accuracy with the increase of step size, which indicates stability. Compared with Strategy \#0, Strategy \#1 and Strategy \#2 both show more 
potential; however, some limitations are observed. Strategy \#1 and \#2 do not reach significance until the step size is more significant than three weeks. The highest testing accuracies from Strategy $\# 1$ and $\# 2$ are $71.57 \%\left(9^{\text {th }}\right.$ week, close to 60$)$ and $73.31 \%\left(6^{\text {th }}\right.$ week, close to 40), both significantly higher than Strategy \#0 does. 


\begin{tabular}{|c|c|c|c|c|c|c|c|}
\hline \multirow{3}{*}{$\begin{array}{c}\text { Step Size } \\
44 \\
\text { features }\end{array}$} & \multicolumn{3}{|l|}{ accuracy\% } & \multicolumn{4}{|c|}{ \# of selected features } \\
\hline & Without FS & ERFS & AHCFS & & ERFS & AHCFS & \\
\hline & & & Step \#1 & Step \#2 & & Step \#1 & Step \#2 \\
\hline \multicolumn{8}{|l|}{$1^{\text {st }}$ week } \\
\hline Mean & $55.66 \%$ & $53.65 \%$ & $53.65 \%$ & $51.49 \%$ & 7 & 2 & 4 \\
\hline SD & $5.04 \%$ & $1.85 \%$ & $3.32 \%$ & $1.94 \%$ & 3.83 & 0.71 & 2.12 \\
\hline \multicolumn{8}{|l|}{$2^{\text {nd }}$ week } \\
\hline Mean & $59.76 \%$ & $57.24 \%$ & $55.11 \%$ & $57.25 \%$ & 24 & 8 & 11 \\
\hline SD & $1.97 \%$ & $3.07 \%$ & $1.48 \%$ & $2.82 \%$ & 14.34 & 6.54 & 10.16 \\
\hline \multicolumn{8}{|l|}{$3^{\text {rd }}$ week } \\
\hline Mean & $60.61 \%$ & $59.02 \%$ & $54.11 \%$ & $60.48 \%$ & 22 & 9 & 20 \\
\hline SD & $1.66 \%$ & $1.21 \%$ & $2.79 \%$ & $9.62 \%$ & 6.22 & 4.95 & 10.23 \\
\hline \multicolumn{8}{|l|}{$4^{\text {th }}$ week } \\
\hline Mean & $61.62 \%$ & $62.69 \%$ & $62.14 \%$ & $64.67 \%$ & 9 & 9 & 16 \\
\hline SD & $2.53 \%$ & $4.62 \%$ & $5.03 \%$ & $4.88 \%$ & 3.49 & 5.31 & 8.14 \\
\hline \multicolumn{8}{|l|}{$5^{\text {th }}$ week } \\
\hline Mean & $64.01 \%$ & $63.74 \%$ & $66.93 \%$ & $68.65 \%$ & 22 & 12 & 15 \\
\hline SD & $2.22 \%$ & $2.09 \%$ & $3.54 \%$ & $4.14 \%$ & 10.16 & 1.48 & 3.27 \\
\hline \multicolumn{8}{|l|}{$6^{\text {th }}$ week } \\
\hline Mean & $66.40 \%$ & $66.81 \%$ & $67.73 \%$ & $73.31 \%$ & 22 & 15 & 20 \\
\hline SD & $1.08 \%$ & $3.91 \%$ & $4.82 \%$ & $2.58 \%$ & 15.07 & 0.43 & 0.83 \\
\hline \multicolumn{8}{|l|}{$7^{\text {th }}$ week } \\
\hline Mean & $67.46 \%$ & $62.81 \%$ & $66.27 \%$ & $71.84 \%$ & 18 & 10 & 22 \\
\hline SD & $1.35 \%$ & $5.50 \%$ & $2.11 \%$ & $2.36 \%$ & 13.06 & 3.49 & 5.17 \\
\hline \multicolumn{8}{|l|}{$8^{\text {th }}$ week } \\
\hline Mean & $67.19 \%$ & $67.74 \%$ & $68.12 \%$ & $72.90 \%$ & 8 & 13 & 26 \\
\hline SD & $1.17 \%$ & $2.87 \%$ & $4.46 \%$ & $3.85 \%$ & 0.83 & 2.45 & 11.43 \\
\hline \multicolumn{8}{|l|}{$9^{\text {th }}$ week } \\
\hline Mean & $69.05 \%$ & $71.57 \%$ & $66.40 \%$ & $70.38 \%$ & 6 & 8 & 11 \\
\hline SD & $1.55 \%$ & $4.48 \%$ & $3.49 \%$ & $7.81 \%$ & 2.49 & 4.26 & 5.26 \\
\hline
\end{tabular}

Table 4. 1 Testing (OOS) accuracies for 2007-2017 
Using testing accuracies of Strategy $\# 0$ as a baseline, Table 4.2 reflects the absolute (abs) and relative (real) improvement on testing accuracies by Strategy \#1 and Strategy \#2 (Step \#1 and Step \#2):

\begin{tabular}{|c|c|c|c|c|c|c|}
\hline & Abs & & & Real & & \\
\hline & ERFS & AHCFS & & ERFS & AHCFS & \\
\hline $1^{\text {st }}$ & $-2.01 \%$ & $-2.01 \%$ & $4.16 \%$ & $3.61 \%$ & $-3.61 \%$ & $-7.48 \%$ \\
\hline $2^{\text {nd }}$ & $-2.52 \%$ & $-4.66 \%$ & $2.52 \%$ & $4.22 \%$ & $-7.79 \%$ & $-4.22 \%$ \\
\hline $3^{\text {rd }}$ & $-1.59 \%$ & $-6.50 \%$ & $0.13 \%$ & $2.63 \%$ & $10.73 \%$ & $-0.22 \%$ \\
\hline $4^{\text {th }}$ & $1.07 \%$ & $0.52 \%$ & $3.05 \%$ & $1.73 \%$ & $0.84 \%$ & $4.94 \%$ \\
\hline $5^{\text {th }}$ & $-0.26 \%$ & $2.92 \%$ & $4.64 \%$ & $0.41 \%$ & $4.56 \%$ & $7.25 \%$ \\
\hline $6^{\text {th }}$ & $0.40 \%$ & $1.33 \%$ & $6.91 \%$ & $0.61 \%$ & $2.00 \%$ & $10.41 \%$ \\
\hline $7^{\text {th }}$ & $-4.65 \%$ & $-1.19 \%$ & $4.38 \%$ & $6.90 \%$ & $-1.77 \%$ & $6.49 \%$ \\
\hline $8^{\text {th }}$ & $0.54 \%$ & $0.93 \%$ & $5.71 \%$ & $0.81 \%$ & $1.38 \%$ & $8.50 \%$ \\
\hline $9^{\text {th }}$ & $2.52 \%$ & $-2.66 \%$ & $1.33 \%$ & $3.65 \%$ & $-3.85 \%$ & $1.92 \%$ \\
\hline
\end{tabular}

Table 4. 2 Improvement by ERFS vs. by AHCFS (baseline: no feature selection)

* Absolute improvement: $A_{a b s}=A_{\# 1 / \# 2}-A_{\# 0}$,

* Absolute improvement: $A_{\text {rel }}=\left(A_{\# 1 / \# 2}-A_{\# 0}\right) / A_{\# 0}$,

$\mathrm{A}_{\text {\#0 }}$ is testing accuracy from Strategy \#0;

$A_{\# 1 / \# 2}$ is the testing accuracy from Strategy \#1 or \#2. 
Without FS vs. ERFS

Compared to Strategy \#0 (Without FS), Strategy \#1 (ERFS) is not very stable or effective. in $1^{\text {st }}, 2^{\text {nd }}, 3^{\text {rd }}, 5^{\text {th }}, 6^{\text {th }}, 5$ out of 9 weekly average, Strategy $\# 1$ underperforms Strategy $\# 0$. For small step sizes (<21 days), Strategy \#1 does not work better, and for large step size, Strategy \#1 sometimes fails to improve testing accuracies. The rate of effectively improved cases is $31.8 \%$, the highest improved accuracy is $2.52 \%$ (abs) \& $3.65 \%$ (rel).

\section{Without FS vs. AHCFS}

Compare to Strategy \#0 (Without FS), Strategy \#2 (AHCFS) works much better. Strategy \#2 outperforms 6 out of 9 weekly average. However, it still doesn't work out for small step sizes. The rate of effectively improved cases is $70.5 \%$, and most cases are improved by using Strategy \#2. The highest improved accuracy is $6.91 \%$ (abs) and $10.41 \%$ (rel).

\section{ERFS vs. AHCFS Step \#1 \& Step \#2}

As two similar algorithms, we would like to see if Strategy \#2 Step \#1 works better than Strategy \#1. The answer is positive. Based on Strategy \#0's results, Strategy \#2 Step \#1's rate of effective cases is $38.6 \%$, which is higher than Strategy \#1's, at $31.8 \%$.

Compared to Strategy \#2 Step \#2 (Strategy \#2 Step \#2 equals Strategy \#2), the rates of effectively improved cases, using Strategy \#0's results as a basis, are $31.8 \%$ vs. $70.5 \%$. Numbers of improved weekly averages are $4 / 9$ versus $6 / 9$. The highest improvement is $2.52 \%$ versus $6.91 \%$ (abs). Strategy \#2 Step \#2 is significantly better. 
4.3 Scenario 2: Experiment based on data with sharp changes (2000-2010)

Parameters and settings are as follows:

1. Classification method - SVM
a. Kernel: Radial Basis Function
b. Normalization Method: zscore
c. Input features: 44 , all come from Technical Indicators

2. Training and testing data
a. Total length: 10 years
b. Training/Testing (IS: OOS) Ratio: 7:3
c. Data type: daily S\&P 500 ^ GSPC, prices, and volume
d. Date: $I S=2000-2007 ;$ OOS $=2007-2010$

3. Features selection:
a. Strategy \#0 vs. Strategy \#1 vs. Strategy \#2
b. Strategy \#2 Step \#1: IS': OOS': 2:5; IS' = $2000-2002$, OOS' $^{\prime}=2002-2007$

4. Step size (prediction horizon): from one day to 60 days (2 months)

Figure 4.2 presents the trending of the S\&P500 from 2000 to 2010, and Table 4.3 presents the testing (OOS) accuracies based on the above piece of data (data period 2000-2012). Compared to 2007-2017, 2000-2010 includes some similarities and some significant differences. The training part is similar in some respects: it begins with a downslope that lasts three years, and then shows a more gradual increase until the separation line. The testing part shows a sharp decrease - a significant drop due to the 
subprime mortgage crisis, and then it picks up a little bit just after a crisis. It is a relatively difficult prediction since the testing (unknown) data begins with a big change in direction, which is inconsistent with training data. However, with the small rebound after 2009, the testing data looks like the training data in miniature. However, the trading volume during the drop (2009) in 2007-2017 fluctuates significantly while the trading volume during the drop (2003) in 2000-2010 fluctuates very slightly.

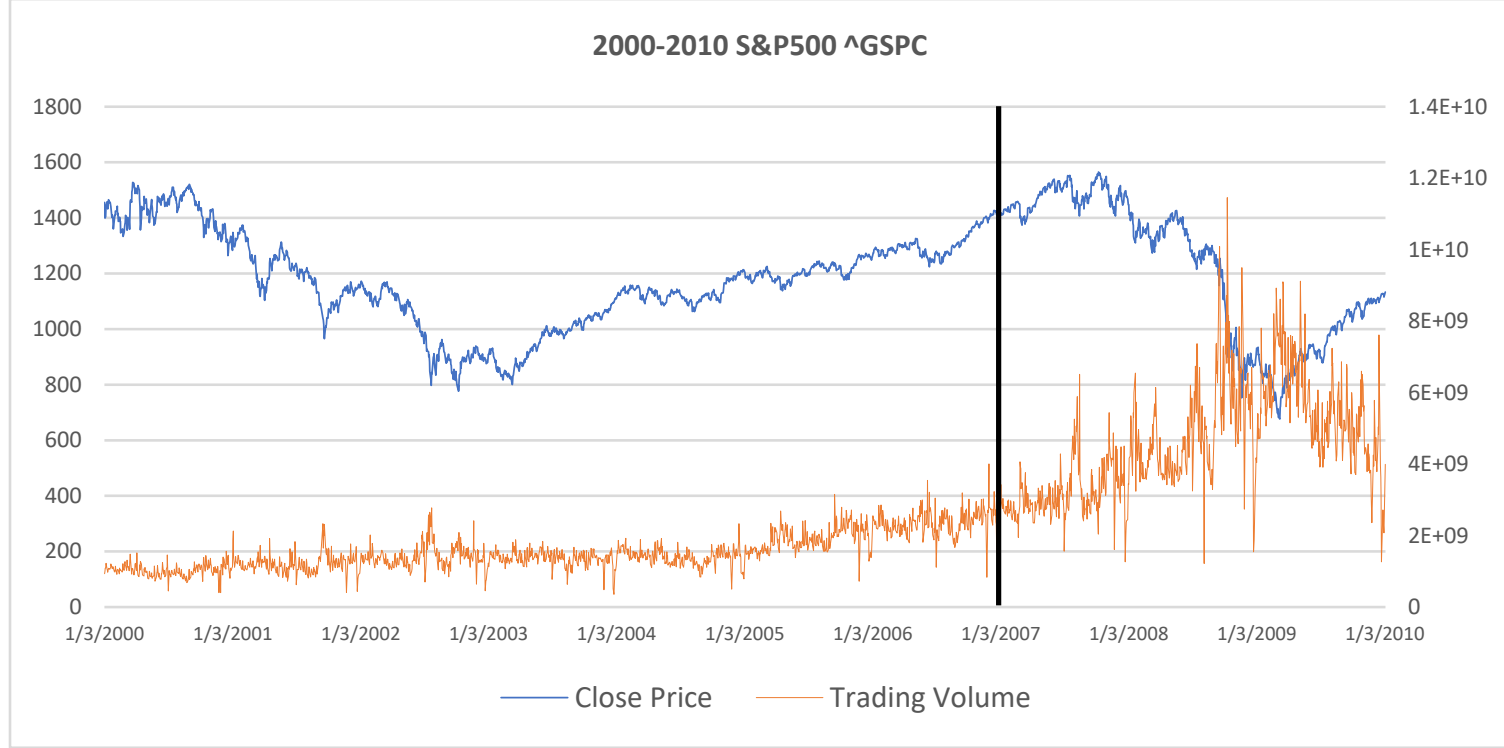

Figure 4. 2 2000-2010 S\&P500^^GSPC trending

In general, Strategy \#0 is ineffective. The testing accuracies vary from $49.51 \%$ to $55.37 \%$, which is as ineffective as a guess with just $50 \%$ accuracy. The standard deviation is equal to or less than $2 \%$, except for the $1^{\text {st }}$ week, which means the fluctuation in testing accuracies is relatively small. The testing accuracies of Strategy \#1 range from $49.16 \%$ to $72.39 \%$, except during the $9^{\text {th }}$ week's $78.75 \%$ (a sudden high). The testing accuracies of Strategy \#2 range from $50.47 \%$ to $77.02 \%$. Both Strategy \#1 and \#2 are within the 
standard deviation at around 4\% (\#1 - 3.64\%, \#2 - 3.98\%), which shows a more substantial fluctuation in testing accuracies than Strategy \#0.

\begin{tabular}{|c|c|c|c|c|c|c|c|}
\hline \multirow{2}{*}{$\begin{array}{l}\text { Step Size } \\
44 \\
\text { features }\end{array}$} & \multicolumn{3}{|l|}{ accuracy\% } & & \multicolumn{3}{|c|}{ \# of selected features } \\
\hline & Without FS & ERFS & $\begin{array}{l}\text { AHCFS } \\
\text { Step \#1 }\end{array}$ & Step \#2 & ERFS & $\begin{array}{l}\text { AHCFS } \\
\text { Step \#1 }\end{array}$ & Step \#2 \\
\hline \multicolumn{8}{|l|}{ 1st week } \\
\hline Mean & $49.51 \%$ & $49.16 \%$ & $50.66 \%$ & $52.31 \%$ & 6 & 5 & 7 \\
\hline SD & $3.80 \%$ & $3.47 \%$ & $1.35 \%$ & $3.55 \%$ & 4.06 & 5.50 & 7.23 \\
\hline \multicolumn{8}{|l|}{ 2nd week } \\
\hline Mean & $52.86 \%$ & $50.08 \%$ & $52.06 \%$ & $50.47 \%$ & 3 & 3 & 6 \\
\hline SD & $1.89 \%$ & $5.00 \%$ & $2.71 \%$ & $2.70 \%$ & 0.50 & 2.06 & 3.96 \\
\hline \multicolumn{8}{|l|}{ 3rd week } \\
\hline Mean & $54.31 \%$ & $56.71 \%$ & $57.64 \%$ & $60.43 \%$ & 12 & 6 & 10 \\
\hline SD & $2.16 \%$ & $2.97 \%$ & $5.96 \%$ & $5.34 \%$ & 15.08 & 3.64 & 4.71 \\
\hline \multicolumn{8}{|l|}{ 4th week } \\
\hline Mean & $55.37 \%$ & $60.97 \%$ & $60.70 \%$ & $66.39 \%$ & 6 & 13 & 19 \\
\hline SD & $1.63 \%$ & $4.61 \%$ & $5.07 \%$ & $6.63 \%$ & 1.09 & 1.30 & 3.54 \\
\hline \multicolumn{8}{|l|}{ 5th week } \\
\hline Mean & $53.51 \%$ & $66.94 \%$ & $70.12 \%$ & $68.13 \%$ & 22 & 12 & 15 \\
\hline SD & $2.12 \%$ & $2.19 \%$ & $2.83 \%$ & $1.06 \%$ & 10.16 & 1.48 & 3.27 \\
\hline \multicolumn{8}{|l|}{ 6th week } \\
\hline Mean & $51.39 \%$ & $65.47 \%$ & $68.93 \%$ & $71.06 \%$ & 6 & 9 & 14 \\
\hline SD & $0.82 \%$ & $3.06 \%$ & $4.03 \%$ & $5.35 \%$ & 1.87 & 2.69 & 2.86 \\
\hline \multicolumn{8}{|l|}{ 7th week } \\
\hline Mean & $50.00 \%$ & $69.89 \%$ & $70.96 \%$ & $73.74 \%$ & 7 & 8 & 14 \\
\hline SD & $0.84 \%$ & $3.90 \%$ & $1.93 \%$ & $3.22 \%$ & 1.09 & 1.66 & 0.83 \\
\hline \multicolumn{8}{|l|}{ 8th week } \\
\hline Mean & $49.93 \%$ & $72.39 \%$ & $67.21 \%$ & $69.86 \%$ & 8 & 12 & 16 \\
\hline SD & $1.25 \%$ & $3.79 \%$ & $4.25 \%$ & $2.52 \%$ & 3.08 & 2.12 & 1.41 \\
\hline \multicolumn{8}{|l|}{ 9th week } \\
\hline Mean & $50.07 \%$ & $78.75 \%$ & $73.84 \%$ & $77.02 \%$ & 7 & 12 & 13 \\
\hline SD & $2.26 \%$ & $3.80 \%$ & $4.37 \%$ & $5.45 \%$ & 1.66 & 3.20 & 2.28 \\
\hline
\end{tabular}


Using the testing accuracies of Strategy \#0, Table 4.4 depicts the absolute and relative improvement on testing accuracies by Strategy \#1 and Strategy \#2 (Step \#1 and Step \#2):

\begin{tabular}{|c|c|c|c|c|c|c|}
\hline & Abs & & & Real & & \\
\hline & ERFS & AHCFS & & ERFS & AHCFS & \\
\hline $1^{\text {st }}$ & $-0.35 \%$ & $1.15 \%$ & $2.81 \%$ & $-0.70 \%$ & $2.33 \%$ & $5.67 \%$ \\
\hline $2^{\text {nd }}$ & $-2.78 \%$ & $-0.80 \%$ & $-2.39 \%$ & $-5.27 \%$ & $-1.52 \%$ & $-4.52 \%$ \\
\hline $3^{\text {rd }}$ & $2.40 \%$ & $3.32 \%$ & $6.12 \%$ & $4.42 \%$ & $6.12 \%$ & $11.27 \%$ \\
\hline $4^{\text {th }}$ & $5.59 \%$ & $5.32 \%$ & $11.01 \%$ & $10.10 \%$ & $9.61 \%$ & $19.89 \%$ \\
\hline $5^{\text {th }}$ & $13.42 \%$ & $16.61 \%$ & $14.61 \%$ & $25.08 \%$ & $31.03 \%$ & $27.31 \%$ \\
\hline $6^{\text {th }}$ & $14.07 \%$ & $17.54 \%$ & $19.67 \%$ & 27.39\% & $34.13 \%$ & $38.27 \%$ \\
\hline $7^{\text {th }}$ & $19.89 \%$ & $20.96 \%$ & $23.74 \%$ & 39.79\% & $41.91 \%$ & $47.49 \%$ \\
\hline $8^{\text {th }}$ & $22.45 \%$ & $17.27 \%$ & $19.92 \%$ & $44.97 \%$ & $34.59 \%$ & $39.90 \%$ \\
\hline$t^{\text {th }}$ & 28.68\% & 23.77\% & $26.95 \%$ & $57.29 \%$ & 47.46\% & $53.82 \%$ \\
\hline
\end{tabular}

Table 4. 4 Improvement by ERFS vs. by AHCFS (baseline: no feature selection)

* Absolute improvement: $A_{a b s}=A_{\# 1 / \# 2}-A_{\# 0}$,

* Absolute improvement: $A_{\text {rel }}=\left(A_{\# 1 / \# 2}-A_{\# 0}\right) / A_{\# 0}$,

$A_{\# 0}$ is testing accuracy from Strategy \#0;

$A_{\# 1 / \# 2}$ is the testing accuracy from Strategy \#1 or \#2. 
Without FS vs. ERFS

Compared to Strategy \#0, Strategy \#1 is relatively effective but slightly less stable. In 7 of 9 weekly averages, Strategy \#1 outperforms Strategy \#0. However, it still does not work out for small step sizes and works better in Scenario 1 (only two weekly averages underperform in this case compared to 3 weekly averages underperforming in Scenario 1). The rate of effectively improved cases is $95.5 \%$, and the highest improved accuracy is $28.68 \%$ (abs) \& $57.29 \%$ (rel).

Without FS vs. AHCFS

Compared to Strategy \#0, Strategy \#2 exhibits better performance in effectiveness, but not instability. In 8 out of 9 weekly averages, Strategy \#2 outperforms Strategy \#0. The rate of effectively improved cases is $84.1 \%$, and the highest accuracy is $26.95 \%$ (abs) and $53.82 \%$ (rel).

ERFS vs. AHCFS Step \#1 \& \#2

Compared with Strategy \#1, Strategy \#2 Step \#1 outperforms 6 out of 9 weekly averages. However, the rate of effectively improved cases, using Strategy \#0's results as the basis, is $84.1 \%$, which is slightly lower than Strategy \#1.

Strategy \#2 Step \#2 (Strategy \#2 Step\#2 equals to Strategy \#2, only when we mention Strategy \#1 do we use Strategy \#2 Step \#2 instead of Strategy \#2) outperforms Strategy $\# 1$ in terms of rate of effectively improved cases ( $84.1 \%$ vs. $84.1 \%$ ) and number of improved weekly averages (8/9 vs. 7/9). However, the highest improvement of Strategy \#2 is smaller. 
4.4 Scenario 3: Experiment based on older data (1992-2002)

Parameters and settings are as follows:

1. Classification method - SVM
a. Kernel: Radial Basis Function
b. Normalization Method: zscore
c. Input features: 44 , all come from Technical Indicators

2. Training and testing data
a. Total length: 10 years
b. Training/Testing (IS: OOS) Ratio: 7:3
c. Data type: daily S\&P 500 ^ GSPC, prices, and volume
d. Date: IS = $1992-1999 ;$ OOS $=1999-2002$

3. Features selection:
a. Strategy \#0 vs. Strategy \#1 vs. Strategy \#2
b. Strategy \#2 Step \#1: IS':OOS': 2:5; IS' = $1992-1994$, OOS' $^{\prime} 1994-1999$

4. Step size (prediction horizon): from one day to 60 days (2 months)

Figure 4.3 shows the trending of the S\&P500 from 1992 to 2002, and Table 4.5 is the testing (OOS) accuracies based on the above data (data period 1992-2002). The trend both in the training and testing parts are quite different from those in Scenarios 1 and 2. The training part consists of two pieces of trends - the first piece is a slow increase and the second piece is speeded increase. The testing part also consists of two pieces of trends - the first piece is a sharp increase which is consistent with another piece in the 
training part and the second piece is a significant decrease. Overall, it is correct to say that the pattern of training data is quite different from that of the testing data.

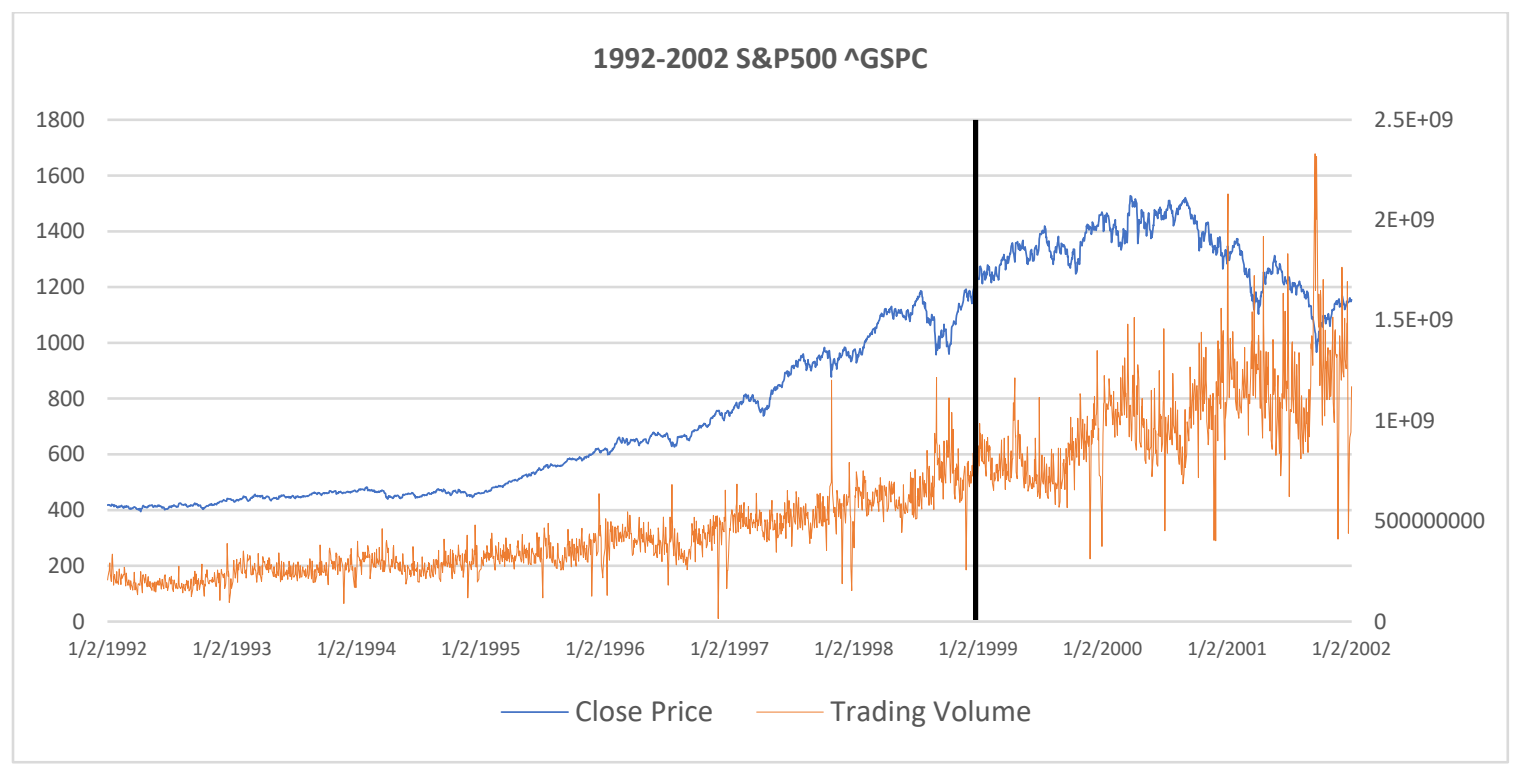

Figure 4.3 1992-2002 S\&P500 ^GSPC trending

In general, Strategy \#0 doesn't work well in this case either. Its testing accuracies vary from $46.20 \%$ to $50.00 \%$, which is roughly equal to guess. It is ineffective. The weekly standard deviation is about $2 \%$. Testing accuracies of Strategy \#1 vary from $49.94 \%$ to $63.52 \%$, which is significant compared with Strategy \#0. Its weekly standard deviation is about $4.5 \%$. The testing accuracies of Strategy \#2 vary from $49.68 \%$ to $75.16 \%$, which is better than Strategy \#1. Its weekly standard deviation is about $2.5 \%$. 


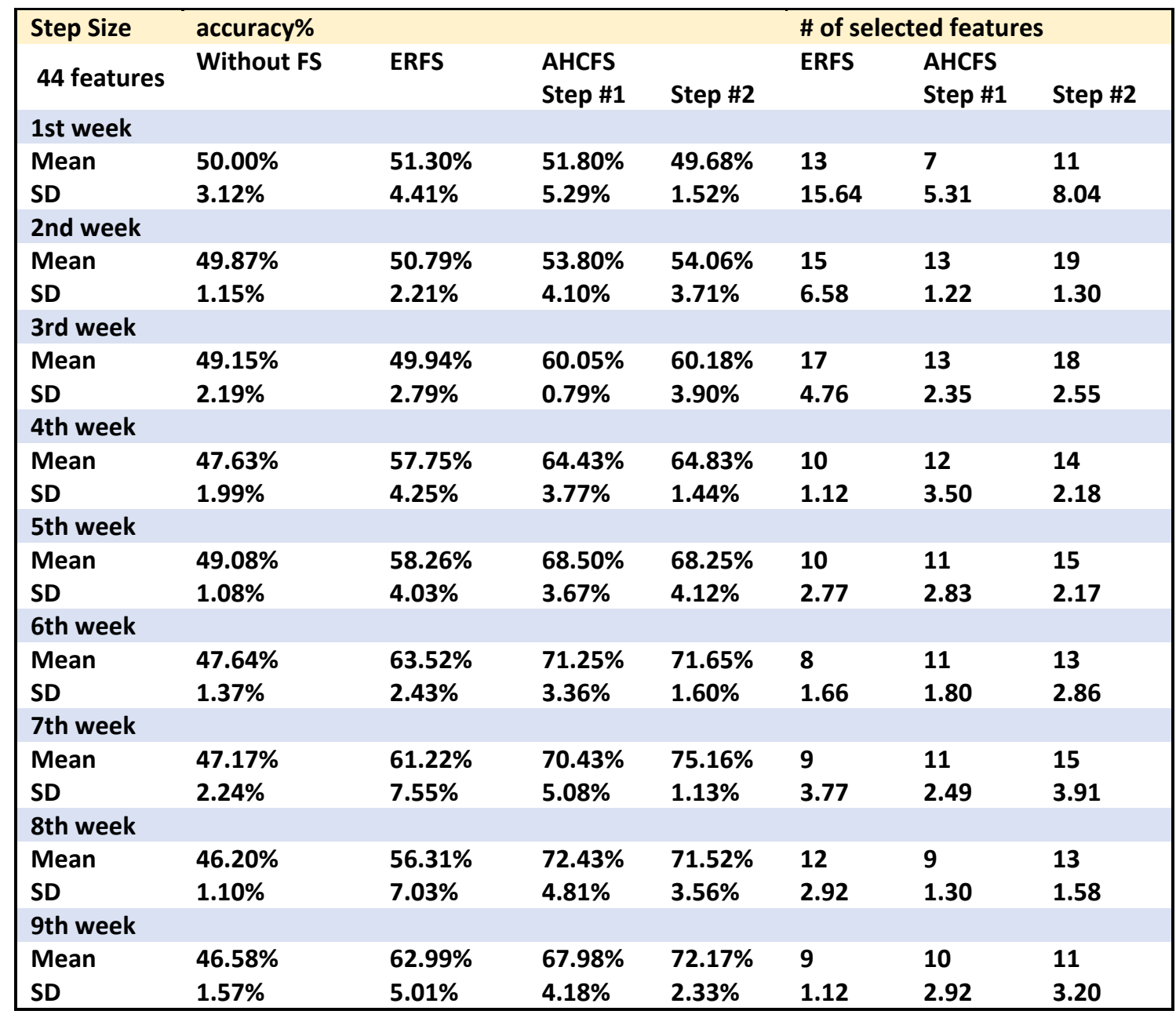

Table 4. 5 Testing (OOS) accuracies for 1992-2002 
Using the testing accuracies of Strategy \#0, Table 4.6 depicts the absolute and relative improvement on testing accuracies by Strategy \#1 and Strategy \#2 (Step \#1 and Step \#2):

\begin{tabular}{llllllll}
\multicolumn{2}{c}{ Abs } & & \multicolumn{3}{l}{ Real } & & \\
& ERFS & AHCFS & & ERFS & AHCFS & \\
& & & & & & \\
$1^{\text {st }}$ & $1.30 \%$ & $1.80 \%$ & $-0.33 \%$ & $2.60 \%$ & $3.60 \%$ & $-0.66 \%$ \\
\hline $2^{\text {nd }}$ & $0.92 \%$ & $3.93 \%$ & $4.19 \%$ & $1.84 \%$ & $7.87 \%$ & $8.40 \%$ \\
\hline $3^{\text {rd }}$ & $0.79 \%$ & $10.91 \%$ & $11.03 \%$ & $1.60 \%$ & $22.19 \%$ & $22.44 \%$ \\
\hline $4^{\text {th }}$ & $10.12 \%$ & $16.79 \%$ & $17.19 \%$ & $21.24 \%$ & $35.26 \%$ & $36.09 \%$ \\
\hline $5^{\text {th }}$ & $9.18 \%$ & $19.41 \%$ & $19.17 \%$ & $18.71 \%$ & $39.56 \%$ & $39.05 \%$ \\
\hline $6^{\text {th }}$ & $15.88 \%$ & $23.61 \%$ & $24.01 \%$ & $33.35 \%$ & $49.57 \%$ & $50.41 \%$ \\
\hline $7^{\text {th }}$ & $14.05 \%$ & $23.26 \%$ & $27.99 \%$ & $29.78 \%$ & $49.30 \%$ & $59.33 \%$ \\
\hline $8^{\text {th }}$ & $10.11 \%$ & $26.23 \%$ & $25.32 \%$ & $21.88 \%$ & $56.78 \%$ & $54.80 \%$ \\
\hline $9^{\text {th }}$ & $16.41 \%$ & $21.39 \%$ & $25.59 \%$ & $35.22 \%$ & $45.92 \%$ & $54.93 \%$
\end{tabular}

Table 4. 6 Improvement by ERFS vs. by AHCFS (baseline: no feature selection)

* Absolute improvement: $A_{a b s}=A_{\# 1 / \# 2}-A_{\# 0}$,

* Absolute improvement: $A_{\text {rel }}=\left(A_{\# 1 / \# 2}-A_{\# 0}\right) / A_{\# 0}$,

$A_{\# 0}$ is the testing accuracy from Strategy \#0;

$A_{\# 1 / \# 2}$ is the testing accuracy from Strategy \#1 or \#2. 
Without FS vs. ERFS

In 9 out of 9 weekly averages, Strategy \#1 outperforms Strategy \#0, however, for small step sizes $\left(1^{\text {st }}, 2^{\text {nd }}\right.$, third weeks $)$, the improvement is so insignificant $(1.3 \%, 0.92 \%, 0.79 \%)$ that it can be disregarded. The rate of effectively improved cases is $88.6 \%$; the highest testing accuracy improvement is $16.41 \%$ (abs) and $35.22 \%$ (rel).

\section{Without FS vs. AHCFS}

In 8 out of 9 weekly averages, Strategy \#2 outperforms Strategy \#0. The $1^{\text {st }}$ week is below average insignificantly $(-0.33 \%)$ as well. The $2^{\text {nd }}$ week's improvement is slightly insignificant (4.19\%) as well. Still, they do not work well for the small step sizes. The rate of effectively improved cases is $93.18 \%$, and the highest improvement is $27.99 \%$ (abs) and $59.33 \%$ (rel).

ERFS vs. AHCFS Step \#1 \& \#2

Compared with Strategy \#2 Step \#1, Strategy \#1 provides a lower rate of effective improved cases ( $88.6 \%$ vs $90.9 \%)$, lower highest improvement ( $16.41 \%$ abs vs. $26.23 \%$ abs), and larger weekly standard deviation (4.41\% vs. $3.89 \%)$.

Compared with Strategy \#2 Step \#2, Strategy \#1 provides a lower rate of effectively improved cases (88.6\% vs. $93.2 \%)$, lower highest improvement (16.41\% abs vs. $27.99 \%$ abs), and a more substantial weekly standard deviation (4.41\% vs. $2.59 \%)$.

Both Strategy \#2 Step \#1 and \#2 outperform Strategy \#1 in this case. 
4.5 Scenario 4: Experiment based on much older data (1960-1970)

Parameters and settings are as follows:

1. Classification method - SVM
a. Kernel: Radial Basis Function
b. Normalization Method: zscore
c. Input features: 44 , all come from Technical Indicators

2. Training and testing data
a. Total length: 10 years
b. Training/Testing (IS: OOS) Ratio: 7:3
c. Data type: daily S\&P 500 ^ GSPC, prices, and volume
d. Date: $I S=2000-2007 ;$ OOS $=2007-2010$

3. Features selection:
a. Strategy \#0 vs. Strategy \#1 vs. Strategy \#2
b. Strategy \#2 Step \#1: IS': OOS': 2:5; IS' = $1960-1967$, OOS' $^{\prime} 1967-1970$

4. Step size (prediction horizon): from one day to 60 days (2 months)

Figure 4.4 demonstrates the trending of the S\&P500 from 1960 to 1970, and Table 4.7 reflects the testing (OOS) accuracies based on the above data. The training part is an increase in general with a sudden drop due to "Flash Crash" in 1962. The testing part also generally increases with a sudden drop due to the 1967 "Sterling Crisis" and a weak end. 


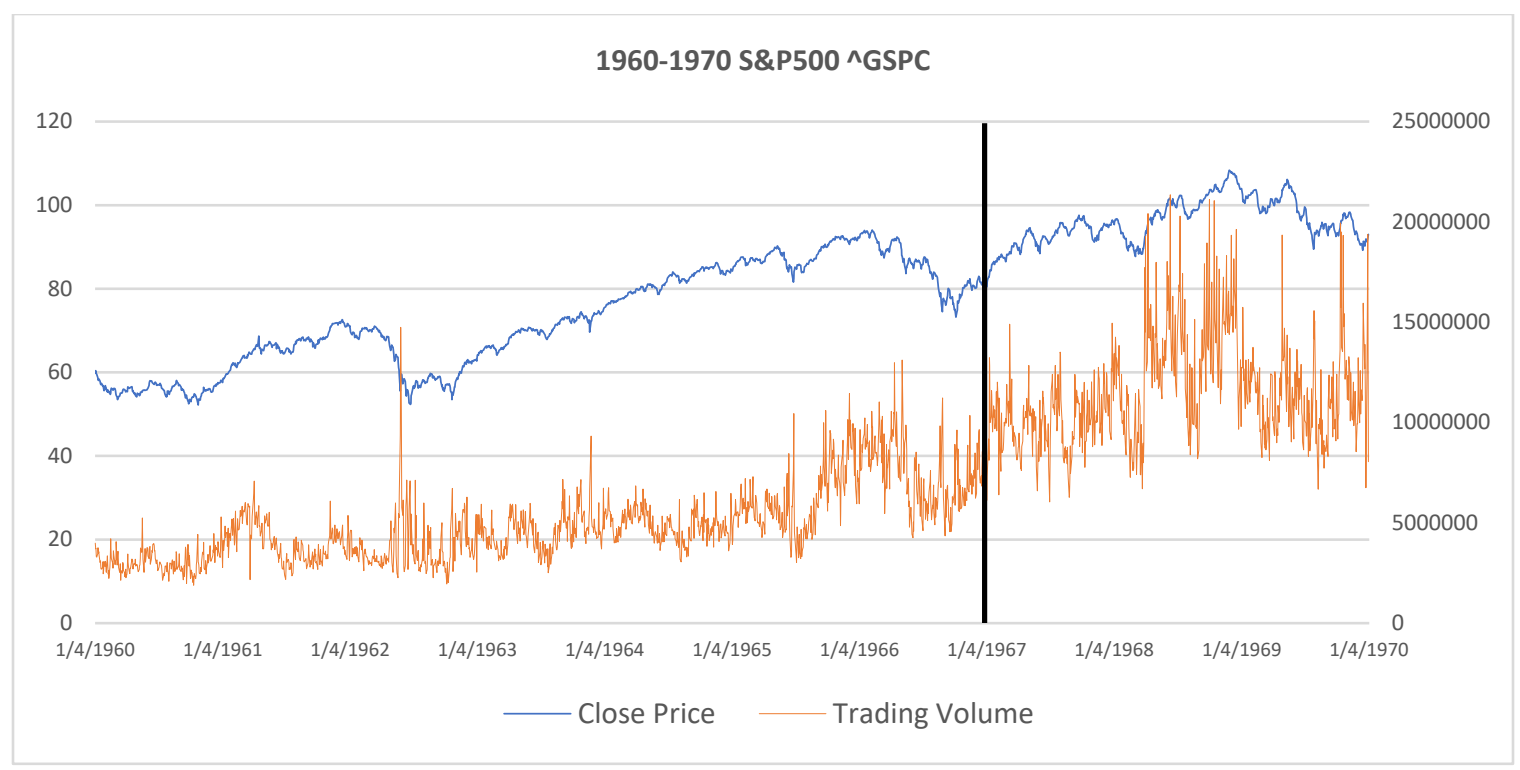

Figure 4. 4 1960-1970 S\&P500 ^GSPC trending

In general, Strategy \#0 works better than it does in the last case; its testing accuracies vary from $53.58 \%$ to $60.03 \%$. Its weekly standard deviation is about $1.5 \%$. Strategy \#1's testing accuracies vary from $54.57 \%$ to $75.83 \%$; its weekly standard deviation is about 4\%. Strategy \#2's testing accuracies vary from $54.56 \%$ to $82.45 \%$ (the highest). Its weekly standard deviation is about 3.5\%, which is lower than Strategy \#1's. 


\begin{tabular}{|c|c|c|c|c|c|c|c|}
\hline \multirow{3}{*}{$\begin{array}{l}\text { Step Size } \\
44 \\
\text { features }\end{array}$} & \multicolumn{4}{|l|}{ accuracy\% } & \multicolumn{3}{|c|}{ \# of selected features } \\
\hline & \multirow[t]{2}{*}{ Without FS } & \multirow[t]{2}{*}{ ERFS } & \multicolumn{2}{|l|}{ AHCFS } & \multirow[t]{2}{*}{ ERFS } & \multicolumn{2}{|l|}{ AHCFS } \\
\hline & & & Step \#1 & Step \#2 & & Step \#1 & Step \#2 \\
\hline \multicolumn{8}{|l|}{ 1st week } \\
\hline Mean & $60.03 \%$ & $60.70 \%$ & $57.54 \%$ & $54.56 \%$ & 37 & 4 & 6 \\
\hline SD & $1.23 \%$ & $2.21 \%$ & $5.12 \%$ & $3.55 \%$ & 7.84 & 3.67 & 3.77 \\
\hline \multicolumn{8}{|l|}{ 2nd week } \\
\hline Mean & $58.30 \%$ & $54.57 \%$ & $56.98 \%$ & $56.04 \%$ & 25 & 4 & 7 \\
\hline SD & $0.82 \%$ & $7.16 \%$ & $4.53 \%$ & $4.62 \%$ & 10.21 & 3.70 & 3.11 \\
\hline \multicolumn{8}{|l|}{ 3rd week } \\
\hline Mean & $57.77 \%$ & $57.37 \%$ & $62.02 \%$ & $61.36 \%$ & 24 & 9 & 14 \\
\hline SD & $1.18 \%$ & $1.68 \%$ & $2.50 \%$ & $4.24 \%$ & 15.12 & 3.00 & 5.41 \\
\hline \multicolumn{8}{|l|}{ 4th week } \\
\hline Mean & $58.70 \%$ & $62.95 \%$ & $65.74 \%$ & $75.04 \%$ & 8 & 11 & 17 \\
\hline SD & $1.40 \%$ & $4.25 \%$ & $4.73 \%$ & $2.47 \%$ & 0.87 & 3.35 & 2.55 \\
\hline \multicolumn{8}{|l|}{ 5th week } \\
\hline Mean & $58.56 \%$ & $66.93 \%$ & $67.07 \%$ & $74.24 \%$ & 9 & 12 & 16 \\
\hline SD & $1.16 \%$ & $4.29 \%$ & $5.64 \%$ & $3.43 \%$ & 3.77 & 2.50 & 5.12 \\
\hline \multicolumn{8}{|l|}{ 6th week } \\
\hline Mean & $55.78 \%$ & $69.45 \%$ & $71.72 \%$ & $79.81 \%$ & 7 & 9 & 13 \\
\hline SD & $0.90 \%$ & $3.97 \%$ & $2.90 \%$ & $3.76 \%$ & 2.28 & 1.66 & 1.58 \\
\hline \multicolumn{8}{|l|}{ 7th week } \\
\hline Mean & $56.70 \%$ & $75.83 \%$ & $81.27 \%$ & $81.27 \%$ & 6 & 13 & 17 \\
\hline SD & $1.46 \%$ & $2.48 \%$ & $3.21 \%$ & $3.54 \%$ & 1.09 & 2.29 & 2.69 \\
\hline \multicolumn{8}{|l|}{ 8th week } \\
\hline Mean & $56.78 \%$ & $74.34 \%$ & $82.17 \%$ & $82.45 \%$ & 6 & 13 & 18 \\
\hline SD & $1.78 \%$ & $6.33 \%$ & $3.22 \%$ & $2.62 \%$ & 1.48 & 1.87 & 1.22 \\
\hline \multicolumn{8}{|l|}{ 9th week } \\
\hline Mean & $53.38 \%$ & $74.37 \%$ & $73.70 \%$ & $79.94 \%$ & 7 & 11 & 15 \\
\hline SD & $1.91 \%$ & $4.54 \%$ & $3.51 \%$ & $3.02 \%$ & 3.96 & 2.95 & 4.09 \\
\hline
\end{tabular}

Table 4. 7 Testing (OOS) accuracies for 1960-1970 
Using the testing accuracies of Strategy \#0, Table 4.8 depicts the absolute and relative improvement on testing accuracies by Strategy \#1 and Strategy \#2 (Step \#1 and Step \#2):

\begin{tabular}{|c|c|c|c|c|c|c|}
\hline & Abs & & & Real & & \\
\hline & ERFS & AHCFS & & ERFS & AHCFS & \\
\hline $1^{\text {st }}$ & $0.66 \%$ & $-2.49 \%$ & $-5.47 \%$ & $1.10 \%$ & $-4.15 \%$ & $-9.11 \%$ \\
\hline $2^{\text {nd }}$ & $-3.73 \%$ & $-1.32 \%$ & $-2.26 \%$ & $-6.40 \%$ & $-2.27 \%$ & $-3.87 \%$ \\
\hline $3^{\text {rd }}$ & $-0.40 \%$ & $4.25 \%$ & $3.59 \%$ & $-0.69 \%$ & $7.36 \%$ & $6.22 \%$ \\
\hline $4^{\text {th }}$ & $4.25 \%$ & 7.05\% & $16.34 \%$ & $7.25 \%$ & $12.00 \%$ & 27.84\% \\
\hline $5^{\text {th }}$ & $8.37 \%$ & $8.50 \%$ & $15.68 \%$ & $14.29 \%$ & $14.52 \%$ & $26.77 \%$ \\
\hline $6^{\text {th }}$ & 13.68\% & $15.94 \%$ & $24.03 \%$ & $24.52 \%$ & $28.58 \%$ & $43.09 \%$ \\
\hline $7^{\text {th }}$ & $19.13 \%$ & $24.57 \%$ & $24.57 \%$ & $33.74 \%$ & $43.33 \%$ & $43.33 \%$ \\
\hline $8^{\text {th }}$ & $17.56 \%$ & $25.40 \%$ & $25.68 \%$ & $30.93 \%$ & $44.73 \%$ & $45.22 \%$ \\
\hline & $8099 \%$ & $20.32 \%$ & $26.56 \%$ & & & \\
\hline
\end{tabular}

Table 4. 8 Improvement by ERFS vs. by AHCFS (baseline: no feature selection)

Without FS vs. ERFS

In 7 out of 9 weekly averages, Strategy \#1 outperforms Strategy \#0, and the same situation occurs. Although improvements appear in small step sizes, the improvements are not significant. The rate of effectively improved cases is $75 \%$, and the highest testing accuracy improvement is $20.99 \%$ (abs) and $39.32 \%$ (rel).

\section{Without FS vs. AHCFS}

In 7 out of 9 weekly averages, Strategy \#2 outperforms Strategy \#0; its performance becomes better when step size becomes more substantial. The rate of effectively 
improved cases is $81.8 \%$, and the highest testing accuracy improvement is $26.56 \%$ (abs) and $49.75 \%$ (rel).

ERFS vs. AHCFS Step \#1 \& Step \#2

Compared with Strategy \#2 Step \#1, Strategy \#1 gives a lower rate of effective improved cases ( $75 \%$ vs. $86.3 \%)$ lower highest improvement ( $20.99 \%$ abs vs. $25.40 \%$ abs), and larger weekly standard deviation (4.10\% vs. $3.93 \%)$.

Compared with Strategy \#2 Step \#2, Strategy \#1 gives a lower rate of effectively improved cases (75\% vs. $81.8 \%$ ), lower highest improvement (20.99\% abs vs. $26.56 \%$ abs), and more substantial weekly standard deviation (4.10\% vs. $3.47 \%)$.

Both Strategy \#2 Step \#1 and \#2 outperform Strategy \#1 in this case. 


\subsection{Others}

One thing needs to be pointed out is that the SVM model without any feature selection algorithm may not get well trained on a 10 -year-long piece of data. That means the results from Strategy \#0 (Without FS) may suffer from an underfitting situation. The extended experiment on the case of FS Strategy \#0 (Without FS) suggests that the SVM prediction model may need more data - a longer piece of data - to be sufficiently trained to give stabilized predicting results, that somehow explains the significant performance differences of Strategy \#0 (Without FS) between in Scenario \#1 and Scenarios \#2, 3, 4. 
Chapter 5 Conclusion and Future Work

This work compared 3 SVM based strategies (algorithms) - \#0: no FS, \#1 ERFS, and \#2 AHCFS on predicting market trends (up or down) of the S\&P 500. Input data consists of 44 features drawn from 24 technical indicators. Additionally, several data groups are collected and experimented within four different periods during which closing price trends and trading volume trends are dissimilar. The prediction horizon is an independent variable ranging from 1 to 60.

When the prediction horizon is smaller than two weeks ( $<14$ days), the prediction accuracy difference from the three strategies is not significant. They are just around $50 \%$, and any of these strategies can outperform others. When the prediction horizon is more significant than three weeks (> 21 days), our target strategy - \#2 always outperforms the other two strategies. When the prediction horizon is between 2 weeks and three weeks (14 days to 21 days), there is 1 case out of every four that our target (strategy \#2) does not significantly outperform (prediction accuracy is like) the other two strategies. (*Due to space constraints, there is no detailed predicting of the results given above. Instead, the weekly averages are shown as a summary to reduce the size of the results table. All the detailed predicting results are listed in Appendix A.)

As a conclusion, our target \#2 strategy-a prediction accuracy based hill climbing feature selection algorithm (AHCFS) - works significantly better when the prediction horizon exceeds three weeks (21 days). 
The average numbers of features selected by strategy $\# 2$ after a prediction horizon larger than three weeks (21 days) are between 10 and 20 (except in scenario 1) and have smaller fluctuation, meaning higher stability compared to strategies \#0 and \#1.

SVM as a favorite machine learning technique is not the only tool we can use. This algorithm can be tested based on other supervised machine learning techniques to fit them better. Moreover, the other non-technical indicators, like macroeconomic indicators, are not tested in this work. The mix of different kind of indicators could a possible way to improve the prediction model. Also, the parameters of indicators could be further optimized to improve the prediction results. 
References

[1] F. X. Diebold and K. Yilmaz, Financial and Macroeconomic Connectedness: A Network Approach to Measurement and Monitoring, New York: Oxford University Press, 2015.

[2] J. Chen, "The Story of Technical Analysis: From the Japanese Rice Markets to Dow Theory to Automated Trading," in Essentials of Technical Analysis for Financial Markets, Hoboken, New Jersey., Wiley \& Sons, Inc., 2010, pp. 11-24.

[3] K. G. Richards, Sideways: Using the Power of Technical Analysis to Profit in Uncertain Times, Toronto and New York: BPS Books, 2011.

[4] Investopedia, "Technical Analysis," INVESTOPEDIA, 0505 2016. [Online]. Available: https://www.investopedia.com/exam-guide/series-7/portfoliomanagement/technicalanalysis.asp?ad=dirN\&qo=investopediaSiteSearch\&qsrc=0\&o=40186.

[5] T. N. Bulkowski, "Fundamental Analysis Summary," in Fundamental Analysis and Position Trading, Hoboken, John Wiley \& Sons, Inc., 2012, pp. 95-99.

[6] R. Rhea, The Dow Theory, www.snowballpublishing.com, 2013. 
[7] S. Matasyan, "The Dow Theory in Technical Analysis," 2013. [Online]. Available: http://www.ifcmarkets.com/en/forex-trading-books/dow-theory-pdf.

[8] J. V. Bergen, "Efficient Market Hypothesis: Is The Stock Market Efficient?," INVESTOPEDIA, 1702 2004. [Online]. Available: https://www.investopedia.com/articles/basics/04/022004.asp.

[9] E. F. Fama, "Random Walks in Stock Market Prices," 1995. [Online]. Available: https://www.cfapubs.org/doi/pdf/10.2469/faj.v51.n1.1861.

[10] A. Plastun, "Behavioral Finance Market Hypotheses," Oxford Scholarship Online, 2017.

[11] M. Santos, "Adaptive Markets Hypothesis: An Evolutionary View About the Relationship Between Environmental Factors and Market Structure," SSRN Electronic Journal, 2015.

[12] P. Rodriguez and S. Rivero, "Using Machine Learning Algorithms To Find Patterns in Stock Prices," SSRN Electronic Journal, 2006.

[13] R. Dash and P. Dash, "A Hybrid Stock Trading Framework Integrating Technical Analysis with Machine Learning Techniques," The Journal of Finance and Data Science, vol. 2, no. 1, pp. 42-57, 2016. 
[14] Y. Shynkevich, T. Mcginnity, S. Coleman, Y. Li and A. Belatreche, "Forecasting stock price directional movements using technical indicators: Investigating window size effects on one-step-ahead forecasting," in 2014 IEEE Conference on Computational Intelligence for Financial Engineering \& Economics (CIFEr), London, UK, 2014.

[15] S. Niaki and S. Hoseinzade, "Forecasting S\&P 500 Index Using Artificial Neural Networks and Design of Experiments," Journal of Industrial Engineering International, vol. 9, no. 1, 2013.

[16] O. Sezer, A. Ozbayoglu and E. Dogdu, "An Artificial Neural Network-based Stock Trading System Using Technical Analysis and Big Data Framework," in The Annual ACM Southeast Conference ACMSE 2017, Kennesaw, Georgia, 2017.

[17] P. Kantavat and B. Kijsirikul, "Combining Technical Analysis and Support Vector Machine For Stock Trading," in 2008 Eighth International Conference on Hybrid Intelligent System, Barcelona, Spain, 2008.

[18] Y. Lin, H. Guo and J. Hu, "An SVM-based Approach For Stock Market Trend Prediction," in The 2013 International Joint Conference on Neural Networks (IJCNN), Dallas, 2013.

[19] S. B. Achelis, Technical analysis from A to Z, New York: McGraw-Hill, 2000. 
[20] I. Guyon and A. Elisseeff, "An Introduction to Variable and Feature selection," Journal of Machine Learning Research, no. 3, pp. 1157-1182, 2003.

[21] L. Ladha and T. Deepa, "Feature Selection Methods and Algorithms," International Journal on Computer Science and Engineering (IJCSE), vol. 3, no. 5, pp. 1787-1797, 2011.

[22] D. W. Aha and R. L. Bankert, "A Comparative Evaluation of Sequential Feature Selection Algorithms," in Learning from Data - Lecture Notes in Statistics vol. 112, New York, Springer, 1996, pp. 199-206.

[23] B. Boser, I. Guyon and V. Vapnik, "A Training Algorithm For Optimal Margin Classifiers," in COLT92 5th Annual Workshop on Computational Learning Theory, New York, 1992.

[24] V. N. Vapnik, Statistical Learning Theory, John Wiley \& Sons, INC., 1998.

[25] I. Steinwart and A. Christmann, "Introduction," in Support Vector Machine (Information Science and Statistics), New York, Springer, 2008, pp. 01-19.

[26] T. Hastie, R. Tibshirani and J. Friedman, "Overview of Supervised Learning," in Elements of Statistical Learning 1st edition, New York, Springer, 2016, pp. 9-39. 
[27] N. Cristianini and J. Shawe-Taylor, An Introduction to Support Vector Machines and Other Kernel-based Learning Methods, Cambridge: Cambridge University Press, 2000.

[28] E. S. Youn, "Feature Selection In Support Vector Machines," 2002.

[29] S. Grigalunas, "List of Technical Indicators," Trading Technologies, 15102015. [Online]. Available: https://www.tradingtechnologies.com/help/xstudy/technical-indicator-definitions/list-of-technical-indicators/.

[30] StockCharts, "Technical Indicators and Overlays," StockCharts.com, 09102017. [Online]. Available: http://stockcharts.com/school/doku.php?id=chart_school\%3Atechnical_indicato rs .

[31] A. T. Alali, "Application of Support Vector Machine in Predicting the Market's Monthly Trend Direction," 2013.

[32] S. Abe, "Feature Selection and Extraction," in Support Vector Machines for Pattern Classification (Advances in Computer Vision and Pattern Recognition) 1st edition, Springer, 2005, pp. 189-199. 
Appendix A Summary Data Sheets

*Due to the size of data, detailed data can only be presented in digital format.

2007-2017

\begin{tabular}{|c|c|c|c|c|c|c|c|}
\hline $\begin{array}{l}\text { Step } \\
\text { Size(days) }\end{array}$ & Without FS & Simple & Improved & & & & \\
\hline $\begin{array}{l}44 \\
\text { features }\end{array}$ & accuracy\% & & Step \#1 & Step \#2 & $\begin{array}{l}\text { \# of } \\
\text { selected } \\
\text { features }\end{array}$ & $\begin{array}{l}\text { \# of } \\
\text { selected } \\
\text { features }\end{array}$ & $\begin{array}{l}\text { \# of } \\
\text { selected } \\
\text { features }\end{array}$ \\
\hline 1 & $47.02 \%$ & $54.30 \%$ & $58.94 \%$ & $49.01 \%$ & 2 & 1 & 2 \\
\hline 2 & $59.33 \%$ & $52.67 \%$ & $54.00 \%$ & $52.00 \%$ & 11 & 2 & 7 \\
\hline 3 & $57.33 \%$ & $51.33 \%$ & $50.67 \%$ & $50.67 \%$ & 10 & 2 & 2 \\
\hline 4 & $58.94 \%$ & $56.29 \%$ & $50.99 \%$ & $54.30 \%$ & 4 & 3 & 5 \\
\hline Mean & $55.66 \%$ & $53.65 \%$ & $53.65 \%$ & $51.49 \%$ & 7 & 2 & 4 \\
\hline Stddev & $5.0425 \%$ & $1.8533 \%$ & $3.3192 \%$ & $1.9382 \%$ & 3.83 & 0.71 & 2.12 \\
\hline 7 & $56.29 \%$ & $54.97 \%$ & $54.97 \%$ & $54.97 \%$ & 1 & 8 & 8 \\
\hline 8 & $58.94 \%$ & $52.32 \%$ & $57.62 \%$ & $55.63 \%$ & 3 & 13 & 17 \\
\hline 9 & $60.67 \%$ & $59.33 \%$ & $54.00 \%$ & $57.33 \%$ & 34 & 1 & 2 \\
\hline 10 & $61.33 \%$ & $60.00 \%$ & $53.33 \%$ & $62.67 \%$ & 40 & 15 & 25 \\
\hline 11 & $61.59 \%$ & $59.60 \%$ & $55.63 \%$ & $55.63 \%$ & 19 & 1 & 1 \\
\hline Mean & $59.76 \%$ & $57.24 \%$ & $55.11 \%$ & $57.25 \%$ & 24 & 8 & 11 \\
\hline Stddev & $1.9671 \%$ & $3.0652 \%$ & $1.4804 \%$ & $2.8218 \%$ & 14.34 & 6.54 & 10.16 \\
\hline 14 & $62.25 \%$ & $57.62 \%$ & $49.01 \%$ & $45.03 \%$ & 7 & 10 & 14 \\
\hline 15 & $61.59 \%$ & $58.28 \%$ & $56.95 \%$ & $54.97 \%$ & 25 & 12 & 15 \\
\hline 16 & $62.00 \%$ & $60.67 \%$ & $54.00 \%$ & $63.33 \%$ & 13 & 1 & 7 \\
\hline 17 & $58.28 \%$ & $60.26 \%$ & $54.30 \%$ & $66.23 \%$ & 30 & 14 & 35 \\
\hline 18 & $58.94 \%$ & $58.28 \%$ & $56.29 \%$ & $72.85 \%$ & 21 & 9 & 21 \\
\hline Mean & $60.61 \%$ & $59.02 \%$ & $54.11 \%$ & $60.48 \%$ & 22 & 9 & 20 \\
\hline Stddev & $1.6620 \%$ & $1.2111 \%$ & $2.7907 \%$ & $9.6240 \%$ & 6.22 & 4.95 & 10.23 \\
\hline 21 & $63.58 \%$ & $63.58 \%$ & $58.94 \%$ & $58.94 \%$ & 44 & 3 & 6 \\
\hline 22 & $60.00 \%$ & $59.33 \%$ & $55.33 \%$ & $58.67 \%$ & 6 & 1 & 4 \\
\hline 23 & $65.33 \%$ & $71.33 \%$ & $60.67 \%$ & $67.33 \%$ & 15 & 7 & 15 \\
\hline 24 & $60.93 \%$ & $58.94 \%$ & $66.89 \%$ & $68.21 \%$ & 9 & 12 & 27 \\
\hline 25 & $58.28 \%$ & $60.26 \%$ & $68.87 \%$ & $70.20 \%$ & 7 & 15 & 16 \\
\hline Mean & $61.62 \%$ & $62.69 \%$ & $62.14 \%$ & $64.67 \%$ & 9 & 9 & 16 \\
\hline Stddev & $2.5254 \%$ & $4.6195 \%$ & $5.0324 \%$ & $4.8800 \%$ & 3.49 & 5.31 & 8.14 \\
\hline 28 & $63.58 \%$ & $62.25 \%$ & $69.54 \%$ & $66.89 \%$ & 14 & 15 & 23 \\
\hline 29 & $60.67 \%$ & $60.67 \%$ & $60.00 \%$ & $61.33 \%$ & 33 & 14 & 18 \\
\hline 30 & $66.67 \%$ & $66.67 \%$ & $69.33 \%$ & $70.67 \%$ & 22 & 12 & 12 \\
\hline 31 & $66.23 \%$ & $64.24 \%$ & $68.21 \%$ & $72.19 \%$ & 6 & 10 & 19 \\
\hline 32 & $62.91 \%$ & $64.90 \%$ & $67.55 \%$ & $72.19 \%$ & 28 & 13 & 12 \\
\hline
\end{tabular}




\begin{tabular}{|c|c|c|c|c|c|c|c|}
\hline Mean & $64.01 \%$ & $63.74 \%$ & $66.93 \%$ & $68.65 \%$ & 22 & 12 & 15 \\
\hline Stddev & $2.2151 \%$ & $2.0891 \%$ & $3.5390 \%$ & $4.1402 \%$ & 10.16 & 1.48 & 3.27 \\
\hline 35 & $64.90 \%$ & $59.60 \%$ & $71.52 \%$ & $72.19 \%$ & 5 & 12 & 20 \\
\hline 36 & $66.00 \%$ & $66.67 \%$ & $60.67 \%$ & $72.67 \%$ & 7 & 15 & 21 \\
\hline 37 & $66.67 \%$ & $71.33 \%$ & $73.33 \%$ & $78.00 \%$ & 7 & 15 & 21 \\
\hline 38 & $68.21 \%$ & $68.21 \%$ & $63.58 \%$ & $73.51 \%$ & 39 & 14 & 20 \\
\hline 39 & $66.23 \%$ & $68.21 \%$ & $69.54 \%$ & $70.20 \%$ & 35 & 15 & 19 \\
\hline Mean & $66.40 \%$ & $66.81 \%$ & $67.73 \%$ & $73.31 \%$ & 22 & 15 & 20 \\
\hline Stddev & $1.0764 \%$ & $3.9079 \%$ & $4.8207 \%$ & $2.5844 \%$ & 15.07 & 0.43 & 0.83 \\
\hline 42 & $68.87 \%$ & $61.59 \%$ & $68.21 \%$ & $72.85 \%$ & 6 & 4 & 9 \\
\hline 43 & $65.33 \%$ & $53.33 \%$ & $64.00 \%$ & $68.67 \%$ & 6 & 8 & 19 \\
\hline 44 & $66.67 \%$ & $64.67 \%$ & $68.67 \%$ & $70.00 \%$ & 5 & 14 & 29 \\
\hline 45 & $67.55 \%$ & $64.24 \%$ & $63.58 \%$ & $75.50 \%$ & 27 & 12 & 23 \\
\hline 46 & $68.87 \%$ & $70.20 \%$ & $66.89 \%$ & $72.19 \%$ & 35 & 5 & 15 \\
\hline Mean & $67.46 \%$ & $62.81 \%$ & $66.27 \%$ & $71.84 \%$ & 18 & 10 & 22 \\
\hline Stddev & $1.3535 \%$ & $5.5023 \%$ & $2.1122 \%$ & $2.3648 \%$ & 13.06 & 3.49 & 5.17 \\
\hline 49 & $66.00 \%$ & $72.67 \%$ & $66.67 \%$ & $66.67 \%$ & 7 & 11 & 19 \\
\hline 50 & $66.00 \%$ & $68.67 \%$ & $66.67 \%$ & $76.00 \%$ & 7 & 15 & 45 \\
\hline 51 & $68.21 \%$ & $67.55 \%$ & $61.59 \%$ & $70.86 \%$ & 7 & 9 & 21 \\
\hline 52 & $68.87 \%$ & $64.90 \%$ & $74.83 \%$ & $77.48 \%$ & 9 & 15 & 22 \\
\hline 53 & $66.89 \%$ & $64.90 \%$ & $70.86 \%$ & $73.51 \%$ & 8 & 13 & 15 \\
\hline Mean & $67.19 \%$ & $67.74 \%$ & $68.12 \%$ & $72.90 \%$ & 8 & 13 & 26 \\
\hline Stddev & $1.1666 \%$ & $2.8737 \%$ & $4.4603 \%$ & $3.8471 \%$ & 0.83 & 2.45 & 11.43 \\
\hline 56 & $70.00 \%$ & $66.67 \%$ & $64.00 \%$ & $64.67 \%$ & 3 & 8 & 15 \\
\hline 57 & $66.00 \%$ & $70.67 \%$ & $67.33 \%$ & $72.67 \%$ & 5 & 10 & 13 \\
\hline 58 & $69.54 \%$ & $78.81 \%$ & $70.20 \%$ & $78.15 \%$ & 10 & 10 & 12 \\
\hline 59 & $70.20 \%$ & $74.17 \%$ & $60.93 \%$ & $58.28 \%$ & 4 & 1 & 2 \\
\hline 60 & $69.54 \%$ & $67.55 \%$ & $69.54 \%$ & $78.15 \%$ & 4 & 12 & 16 \\
\hline Mean & $69.05 \%$ & $71.57 \%$ & $66.40 \%$ & $70.38 \%$ & 6 & 8 & 11 \\
\hline Stddev & $1.5490 \%$ & $4.4762 \%$ & $3.4886 \%$ & $7.8095 \%$ & 2.49 & 4.26 & 5.26 \\
\hline
\end{tabular}


2000-2010

\begin{tabular}{|c|c|c|c|c|c|c|c|}
\hline $\begin{array}{l}\text { Step } \\
\text { Size(days) }\end{array}$ & Without FS & Simple & Improved & & & & \\
\hline $\begin{array}{l}44 \\
\text { features }\end{array}$ & accuracy\% & & Step \#1 & Step \#2 & $\begin{array}{l}\text { \# of } \\
\text { selected } \\
\text { features }\end{array}$ & $\begin{array}{l}\text { \# of } \\
\text { selected } \\
\text { features }\end{array}$ & $\begin{array}{l}\text { \# of } \\
\text { selected } \\
\text { features }\end{array}$ \\
\hline 1 & $48.34 \%$ & $54.30 \%$ & $52.98 \%$ & $56.29 \%$ & 2 & 1 & 2 \\
\hline 2 & $47.02 \%$ & $49.67 \%$ & $49.67 \%$ & $54.97 \%$ & 2 & 2 & 3 \\
\hline 3 & $56.00 \%$ & $44.67 \%$ & $50.00 \%$ & $47.33 \%$ & 9 & 1 & 2 \\
\hline 4 & $46.67 \%$ & $48.00 \%$ & $50.00 \%$ & $50.67 \%$ & 11 & 14 & 19 \\
\hline Mean & $49.51 \%$ & $49.16 \%$ & $50.66 \%$ & $52.31 \%$ & 6 & 5 & 7 \\
\hline Stddev & $3.8001 \%$ & $3.4735 \%$ & $1.3450 \%$ & $3.5489 \%$ & 4.06 & 5.50 & 7.23 \\
\hline 7 & $52.98 \%$ & $50.99 \%$ & $53.64 \%$ & $50.33 \%$ & 5 & 1 & 4 \\
\hline 8 & $50.33 \%$ & $51.66 \%$ & $47.02 \%$ & $49.01 \%$ & 2 & 1 & 3 \\
\hline 9 & $51.66 \%$ & $40.40 \%$ & $54.97 \%$ & $46.36 \%$ & 3 & 6 & 13 \\
\hline 10 & $56.00 \%$ & $52.67 \%$ & $52.67 \%$ & $54.00 \%$ & 2 & 2 & 4 \\
\hline 11 & $53.33 \%$ & $54.67 \%$ & $52.00 \%$ & $52.67 \%$ & 3 & 1 & 5 \\
\hline Mean & $52.86 \%$ & $50.08 \%$ & $52.06 \%$ & $50.47 \%$ & 3 & 3 & 6 \\
\hline Stddev & $1.8944 \%$ & $4.9960 \%$ & $2.7103 \%$ & $2.6966 \%$ & 0.50 & 2.06 & 3.96 \\
\hline 14 & $52.32 \%$ & $51.66 \%$ & $51.66 \%$ & $54.97 \%$ & 1 & 1 & 2 \\
\hline 15 & $56.95 \%$ & $56.95 \%$ & $56.95 \%$ & $53.64 \%$ & 1 & 1 & 2 \\
\hline 16 & $52.67 \%$ & $60.67 \%$ & $50.67 \%$ & $61.33 \%$ & 4 & 3 & 13 \\
\hline 17 & $52.67 \%$ & $56.00 \%$ & $64.67 \%$ & $65.33 \%$ & 5 & 8 & 10 \\
\hline 18 & $56.95 \%$ & $58.28 \%$ & $64.24 \%$ & $66.89 \%$ & 38 & 10 & 14 \\
\hline Mean & $54.31 \%$ & $56.71 \%$ & $57.64 \%$ & $60.43 \%$ & 12 & 6 & 10 \\
\hline Stddev & $2.1609 \%$ & $2.9740 \%$ & $5.9636 \%$ & $5.3381 \%$ & 15.08 & 3.64 & 4.71 \\
\hline 21 & $57.62 \%$ & $52.98 \%$ & $52.98 \%$ & $70.86 \%$ & 1 & 1 & 15 \\
\hline 22 & $56.29 \%$ & $62.91 \%$ & $60.26 \%$ & $69.54 \%$ & 4 & 11 & 13 \\
\hline 23 & $55.33 \%$ & $66.00 \%$ & $67.33 \%$ & $70.67 \%$ & 7 & 14 & 20 \\
\hline 24 & $52.67 \%$ & $64.00 \%$ & $58.00 \%$ & $53.33 \%$ & 6 & 14 & 22 \\
\hline 25 & $54.97 \%$ & $58.94 \%$ & $64.90 \%$ & $67.55 \%$ & 6 & 14 & 21 \\
\hline Mean & $55.37 \%$ & $60.97 \%$ & $60.70 \%$ & $66.39 \%$ & 6 & 13 & 19 \\
\hline Stddev & $1.6349 \%$ & $4.6091 \%$ & $5.0739 \%$ & $6.6333 \%$ & 1.09 & 1.30 & 3.54 \\
\hline 28 & $54.97 \%$ & $64.90 \%$ & $70.86 \%$ & $66.23 \%$ & 9 & 13 & 18 \\
\hline 29 & $54.30 \%$ & $64.24 \%$ & $66.89 \%$ & $68.21 \%$ & 7 & 8 & 21 \\
\hline 30 & $49.33 \%$ & $66.67 \%$ & $74.67 \%$ & $68.00 \%$ & 7 & 13 & 18 \\
\hline 31 & $54.00 \%$ & $69.33 \%$ & $67.33 \%$ & $69.33 \%$ & 4 & 10 & 14 \\
\hline 32 & $54.97 \%$ & $69.54 \%$ & $70.86 \%$ & $68.87 \%$ & 5 & 9 & 12 \\
\hline Mean & $53.51 \%$ & $66.94 \%$ & $70.12 \%$ & $68.13 \%$ & 6 & 10 & 16 \\
\hline Stddev & $2.1242 \%$ & $2.1910 \%$ & $2.8279 \%$ & $1.0634 \%$ & 1.30 & 1.87 & 3.49 \\
\hline 35 & $51.66 \%$ & $61.59 \%$ & $70.20 \%$ & $74.83 \%$ & 4 & 7 & 18 \\
\hline 36 & $52.32 \%$ & $70.20 \%$ & $66.23 \%$ & $64.90 \%$ & 8 & 7 & 12 \\
\hline
\end{tabular}




\begin{tabular}{|l|l|l|l|l|l|l|l|}
\hline 37 & $50.00 \%$ & $63.33 \%$ & $74.67 \%$ & $76.67 \%$ & 7 & 8 & 16 \\
\hline 38 & $52.00 \%$ & $64.67 \%$ & $70.67 \%$ & $74.67 \%$ & 6 & 6 & 10 \\
\hline 39 & $50.99 \%$ & $67.55 \%$ & $62.91 \%$ & $64.24 \%$ & 3 & 13 & 17 \\
\hline Mean & $\mathbf{5 1 . 3 9 \%}$ & $\mathbf{6 5 . 4 7 \%}$ & $\mathbf{6 8 . 9 3 \%}$ & $\mathbf{7 1 . 0 6 \%}$ & $\mathbf{6}$ & $\mathbf{9}$ & $\mathbf{1 4}$ \\
\hline Stddev & $\mathbf{0 . 8 2 3 8 \%}$ & $\mathbf{3 . 0 6 4 5 \%}$ & $\mathbf{4 . 0 2 6 0} \%$ & $\mathbf{5 . 3 5 0 9 \%}$ & $\mathbf{1 . 8 7}$ & $\mathbf{2 . 6 9}$ & $\mathbf{2 . 8 6}$ \\
\hline 42 & $49.67 \%$ & $70.20 \%$ & $70.86 \%$ & $70.20 \%$ & 5 & 7 & 12 \\
\hline 43 & $50.99 \%$ & $72.85 \%$ & $73.51 \%$ & $69.54 \%$ & 5 & 8 & 14 \\
\hline 44 & $49.33 \%$ & $69.33 \%$ & $71.33 \%$ & $75.33 \%$ & 7 & 10 & 13 \\
\hline 45 & $49.01 \%$ & $62.91 \%$ & $67.55 \%$ & $76.82 \%$ & 8 & 6 & 13 \\
\hline 46 & $50.99 \%$ & $74.17 \%$ & $71.52 \%$ & $76.82 \%$ & 7 & 6 & 15 \\
\hline Mean & $\mathbf{5 0 . 0 0 \%}$ & $\mathbf{6 9 . 8 9 \%}$ & $\mathbf{7 0 . 9 6 \%}$ & $\mathbf{7 3 . 7 4 \%}$ & $\mathbf{7}$ & $\mathbf{8}$ & $\mathbf{1 4}$ \\
\hline Stddev & $\mathbf{0 . 8 3 8 4 \%}$ & $\mathbf{3 . 9 0 2 7 \%}$ & $\mathbf{1 . 9 2 8 7 \%}$ & $\mathbf{3 . 2 1 6 7 \%}$ & $\mathbf{1 . 0 9}$ & $\mathbf{1 . 6 6}$ & $\mathbf{0 . 8 3}$ \\
\hline 49 & $49.01 \%$ & $72.19 \%$ & $64.24 \%$ & $70.86 \%$ & 6 & 8 & 16 \\
\hline 50 & $49.33 \%$ & $76.00 \%$ & $70.00 \%$ & $70.67 \%$ & 13 & 9 & 14 \\
\hline 51 & $50.00 \%$ & $76.67 \%$ & $70.67 \%$ & $72.00 \%$ & 6 & 11 & 16 \\
\hline 52 & $49.01 \%$ & $70.86 \%$ & $60.26 \%$ & $64.90 \%$ & 8 & 14 & 18 \\
\hline 53 & $52.32 \%$ & $66.23 \%$ & $70.86 \%$ & $70.86 \%$ & 5 & 14 & 16 \\
\hline Mean & $\mathbf{4 9 . 9 3 \%}$ & $\mathbf{7 2 . 3 9 \%}$ & $\mathbf{6 7 . 2 1 \%}$ & $\mathbf{6 9 . 8 6 \%}$ & $\mathbf{8}$ & $\mathbf{1 2}$ & $\mathbf{1 6}$ \\
\hline Stddev & $\mathbf{1 . 2 4 6 4 \%}$ & $\mathbf{3 . 7 8 7 0} \%$ & $\mathbf{4 . 2 4 5 6 \%}$ & $\mathbf{2 . 5 2 3 1 \%}$ & $\mathbf{3 . 0 8}$ & $\mathbf{2 . 1 2}$ & $\mathbf{1 . 4 1}$ \\
\hline 56 & $46.36 \%$ & $72.19 \%$ & $78.81 \%$ & $81.46 \%$ & 6 & 6 & 13 \\
\hline 57 & $53.33 \%$ & $81.33 \%$ & $78.00 \%$ & $80.00 \%$ & 8 & 10 & 13 \\
\hline 58 & $50.00 \%$ & $78.00 \%$ & $68.00 \%$ & $68.00 \%$ & 8 & 15 & 17 \\
\hline 59 & $50.99 \%$ & $83.44 \%$ & $69.54 \%$ & $73.51 \%$ & 6 & 14 & 11 \\
\hline 60 & $49.67 \%$ & $78.81 \%$ & $74.83 \%$ & $82.12 \%$ & 4 & 7 & 12 \\
\hline Mean & $\mathbf{5 0 . 0 7 \%}$ & $\mathbf{7 8 . 7 5 \%}$ & $\mathbf{7 3 . 8 4 \%}$ & $\mathbf{7 7 . 0 2} \%$ & $\mathbf{7}$ & $\mathbf{1 2}$ & $\mathbf{1 3}$ \\
\hline Stddev & $\mathbf{2 . 2 5 6 1 \%}$ & $\mathbf{3 . 8 0 4 3} \%$ & $\mathbf{4 . 3 7 2 7} \%$ & $\mathbf{5 . 4 4 5 2} \%$ & $\mathbf{1 . 6 6}$ & $\mathbf{3 . 2 0}$ & $\mathbf{2 . 2 8}$ \\
\hline
\end{tabular}


1992-2002

\begin{tabular}{|c|c|c|c|c|c|c|c|}
\hline $\begin{array}{l}\text { Step } \\
\text { Size(days) }\end{array}$ & Without FS & Simple & Improved & & & & \\
\hline $\begin{array}{l}44 \\
\text { features }\end{array}$ & accuracy\% & & Step \#1 & Step \#2 & $\begin{array}{l}\text { \# of } \\
\text { selected } \\
\text { features }\end{array}$ & $\begin{array}{l}\text { \# of } \\
\text { selected } \\
\text { features }\end{array}$ & $\begin{array}{l}\text { \# of } \\
\text { selected } \\
\text { features }\end{array}$ \\
\hline 1 & $48.03 \%$ & $48.03 \%$ & $47.37 \%$ & $50.66 \%$ & 40 & 2 & 3 \\
\hline 2 & $54.61 \%$ & $50.00 \%$ & $56.58 \%$ & $50.66 \%$ & 8 & 11 & 10 \\
\hline 3 & $50.98 \%$ & $58.82 \%$ & $57.52 \%$ & $50.33 \%$ & 4 & 1 & 6 \\
\hline 4 & $46.41 \%$ & $48.37 \%$ & $45.75 \%$ & $47.06 \%$ & 1 & 13 & 24 \\
\hline Mean & $50.00 \%$ & $51.30 \%$ & $51.80 \%$ & $49.68 \%$ & 13 & 7 & 11 \\
\hline Stddev & $3.1220 \%$ & $4.4051 \%$ & $5.2853 \%$ & $1.5167 \%$ & 15.64 & 5.31 & 8.04 \\
\hline 7 & $48.68 \%$ & $48.68 \%$ & $47.37 \%$ & $48.68 \%$ & 32 & 13 & 15 \\
\hline 8 & $51.97 \%$ & $54.61 \%$ & $50.66 \%$ & $55.92 \%$ & 22 & 13 & 20 \\
\hline 9 & $50.00 \%$ & $48.68 \%$ & $56.58 \%$ & $50.66 \%$ & 17 & 14 & 17 \\
\hline 10 & $49.67 \%$ & $50.33 \%$ & $56.21 \%$ & $56.86 \%$ & 15 & 14 & 18 \\
\hline 11 & $49.02 \%$ & $51.63 \%$ & $58.17 \%$ & $58.17 \%$ & 4 & 11 & 20 \\
\hline Mean & $49.87 \%$ & $50.79 \%$ & $53.80 \%$ & $54.06 \%$ & 15 & 13 & 19 \\
\hline Stddev & $1.1498 \%$ & $2.2069 \%$ & $4.0954 \%$ & $3.7063 \%$ & 6.58 & 1.22 & 1.30 \\
\hline 14 & $48.03 \%$ & $47.37 \%$ & $61.18 \%$ & $54.61 \%$ & 9 & 12 & 17 \\
\hline 15 & $48.03 \%$ & $48.68 \%$ & $59.87 \%$ & $64.47 \%$ & 21 & 9 & 16 \\
\hline 16 & $53.29 \%$ & $55.26 \%$ & $59.87 \%$ & $57.89 \%$ & 16 & 15 & 21 \\
\hline 17 & $47.06 \%$ & $48.37 \%$ & $58.82 \%$ & $64.71 \%$ & 22 & 14 & 15 \\
\hline 18 & $49.34 \%$ & $50.00 \%$ & $60.53 \%$ & $59.21 \%$ & 10 & 14 & 20 \\
\hline Mean & $49.15 \%$ & $49.94 \%$ & $60.05 \%$ & $60.18 \%$ & 17 & 13 & 18 \\
\hline Stddev & $2.1941 \%$ & $2.7931 \%$ & $0.7853 \%$ & $3.9028 \%$ & 4.76 & 2.35 & 2.55 \\
\hline 21 & $44.74 \%$ & $55.92 \%$ & $61.18 \%$ & $64.47 \%$ & 9 & 13 & 18 \\
\hline 22 & $46.05 \%$ & $63.16 \%$ & $65.79 \%$ & $63.16 \%$ & 8 & 15 & 13 \\
\hline 23 & $48.37 \%$ & $50.98 \%$ & $70.59 \%$ & $67.32 \%$ & 9 & 11 & 13 \\
\hline 24 & $50.33 \%$ & $57.52 \%$ & $64.71 \%$ & $65.36 \%$ & 10 & 6 & 11 \\
\hline 25 & $48.68 \%$ & $61.18 \%$ & $59.87 \%$ & $63.82 \%$ & 11 & 14 & 17 \\
\hline Mean & $47.63 \%$ & $57.75 \%$ & $64.43 \%$ & $64.83 \%$ & 10 & 12 & 14 \\
\hline Stddev & $1.9892 \%$ & $4.2489 \%$ & $3.7734 \%$ & $1.4445 \%$ & 1.12 & 3.50 & 2.18 \\
\hline 28 & $48.68 \%$ & $58.55 \%$ & $63.16 \%$ & $71.71 \%$ & 6 & 4 & 14 \\
\hline 29 & $49.34 \%$ & $58.55 \%$ & $68.42 \%$ & $73.68 \%$ & 7 & 11 & 14 \\
\hline 30 & $47.71 \%$ & $53.59 \%$ & $74.51 \%$ & $63.40 \%$ & 13 & 11 & 15 \\
\hline 31 & $50.98 \%$ & $65.36 \%$ & $69.28 \%$ & $68.63 \%$ & 7 & 15 & 18 \\
\hline 32 & $48.68 \%$ & $55.26 \%$ & $67.11 \%$ & $63.82 \%$ & 12 & 7 & 12 \\
\hline Mean & $49.08 \%$ & $58.26 \%$ & $68.50 \%$ & $68.25 \%$ & 10 & 11 & 15 \\
\hline Stddev & $1.0829 \%$ & $4.0328 \%$ & $3.6665 \%$ & $4.1194 \%$ & 2.77 & 2.83 & 2.17 \\
\hline 35 & $47.37 \%$ & $64.47 \%$ & $67.76 \%$ & $71.05 \%$ & 9 & 15 & 17 \\
\hline 36 & $46.05 \%$ & 65.79\% & $67.76 \%$ & $69.74 \%$ & 6 & 13 & 17 \\
\hline
\end{tabular}




\begin{tabular}{|c|c|c|c|c|c|c|c|}
\hline 37 & $46.41 \%$ & $64.71 \%$ & $75.82 \%$ & $73.20 \%$ & 6 & 11 & 13 \\
\hline 38 & $49.67 \%$ & $58.82 \%$ & $74.51 \%$ & $73.86 \%$ & 8 & 8 & 14 \\
\hline 39 & $48.68 \%$ & $63.82 \%$ & $70.39 \%$ & $70.39 \%$ & 10 & 10 & 9 \\
\hline Mean & 47.64\% & 63.52\% & 71.25\% & 71.65\% & 8 & 11 & 13 \\
\hline Stddev & $1.3669 \%$ & $2.4335 \%$ & $3.3625 \%$ & $1.6044 \%$ & 1.66 & 1.80 & 2.86 \\
\hline 42 & $46.05 \%$ & $54.61 \%$ & $75.00 \%$ & $75.66 \%$ & 12 & 15 & 12 \\
\hline 43 & $43.42 \%$ & $51.32 \%$ & $72.37 \%$ & 74.34\% & 15 & 15 & 20 \\
\hline 44 & $47.71 \%$ & $71.24 \%$ & $72.55 \%$ & $75.16 \%$ & 7 & 9 & 14 \\
\hline 45 & $49.34 \%$ & $67.76 \%$ & $60.53 \%$ & $73.68 \%$ & 5 & 12 & 9 \\
\hline 46 & $49.34 \%$ & $61.18 \%$ & $71.71 \%$ & $76.97 \%$ & 10 & 9 & 15 \\
\hline Mean & $47.17 \%$ & $61.22 \%$ & $70.43 \%$ & $75.16 \%$ & 9 & 11 & 15 \\
\hline Stddev & $2.2376 \%$ & $7.5512 \%$ & $5.0765 \%$ & $1.1300 \%$ & 3.77 & 2.49 & 3.91 \\
\hline 49 & $46.05 \%$ & $68.42 \%$ & $66.45 \%$ & $69.74 \%$ & 6 & 9 & 12 \\
\hline 50 & $45.75 \%$ & $59.48 \%$ & $77.78 \%$ & $69.93 \%$ & 7 & 10 & 11 \\
\hline 51 & $44.44 \%$ & $48.37 \%$ & $78.43 \%$ & $78.43 \%$ & 14 & 8 & 12 \\
\hline 52 & $47.37 \%$ & $52.63 \%$ & $70.39 \%$ & $68.42 \%$ & 13 & 11 & 15 \\
\hline 53 & $47.37 \%$ & $52.63 \%$ & $69.08 \%$ & $71.05 \%$ & 14 & 8 & 14 \\
\hline Mean & $46.20 \%$ & $56.31 \%$ & $72.43 \%$ & $71.52 \%$ & 12 & 9 & 13 \\
\hline Stddev & $1.0986 \%$ & $7.0268 \%$ & $4.8120 \%$ & $3.5576 \%$ & 2.92 & 1.30 & 1.58 \\
\hline 56 & $44.08 \%$ & $62.50 \%$ & $70.39 \%$ & $72.37 \%$ & 7 & 8 & 9 \\
\hline 57 & $47.71 \%$ & $55.56 \%$ & $64.05 \%$ & $70.59 \%$ & 10 & 11 & 9 \\
\hline 58 & $47.71 \%$ & $71.24 \%$ & $74.51 \%$ & $76.47 \%$ & 8 & 9 & 16 \\
\hline 59 & $45.39 \%$ & $61.84 \%$ & $67.76 \%$ & $69.74 \%$ & 9 & 6 & 8 \\
\hline 60 & $48.03 \%$ & $63.82 \%$ & $63.16 \%$ & $71.71 \%$ & 7 & 14 & 9 \\
\hline Mean & $46.58 \%$ & $62.99 \%$ & $67.98 \%$ & 72.17\% & 9 & 10 & 11 \\
\hline Stddev & $1.5695 \%$ & $5.0121 \%$ & $4.1760 \%$ & $2.3310 \%$ & 1.12 & 2.92 & 3.20 \\
\hline
\end{tabular}


1960-1970

\begin{tabular}{|c|c|c|c|c|c|c|c|}
\hline $\begin{array}{l}\text { Step } \\
\text { Size(days) }\end{array}$ & $\begin{array}{l}\text { Without } \\
\text { FS }\end{array}$ & Simple & Improved & & & & \\
\hline $\begin{array}{c}44 \\
\text { features }\end{array}$ & $\begin{array}{l}\text { accuracy } \\
\%\end{array}$ & & Step \#1 & Step \#2 & $\begin{array}{l}\text { \# of } \\
\text { selected } \\
\text { features }\end{array}$ & $\begin{array}{l}\text { \# of } \\
\text { selected } \\
\text { features }\end{array}$ & $\begin{array}{l}\text { \# of } \\
\text { selected } \\
\text { features }\end{array}$ \\
\hline 1 & $58.28 \%$ & $58.28 \%$ & $60.93 \%$ & $59.60 \%$ & 44 & 1 & 3 \\
\hline 2 & $59.60 \%$ & $59.60 \%$ & $63.58 \%$ & $52.32 \%$ & 44 & 1 & 2 \\
\hline 3 & $61.59 \%$ & $64.24 \%$ & $50.33 \%$ & $50.33 \%$ & 25 & 10 & 10 \\
\hline 4 & $60.67 \%$ & $60.67 \%$ & $55.33 \%$ & $56.00 \%$ & 35 & 4 & 10 \\
\hline Mean & $60.03 \%$ & $60.70 \%$ & $57.54 \%$ & $54.56 \%$ & 37 & 4 & 6 \\
\hline Stddev & $1.2338 \%$ & $2.2131 \%$ & $5.1173 \%$ & $3.5501 \%$ & 7.84 & 3.67 & 3.77 \\
\hline 7 & $58.00 \%$ & $40.67 \%$ & $64.00 \%$ & $58.00 \%$ & 3 & 1 & 2 \\
\hline 8 & $56.95 \%$ & $56.95 \%$ & $58.94 \%$ & $63.58 \%$ & 31 & 5 & 9 \\
\hline 9 & $58.28 \%$ & $55.63 \%$ & $50.33 \%$ & $49.67 \%$ & 7 & 1 & 2 \\
\hline 10 & $58.94 \%$ & $58.94 \%$ & $56.95 \%$ & $54.97 \%$ & 32 & 10 & 10 \\
\hline 11 & $59.33 \%$ & $60.67 \%$ & $54.67 \%$ & $54.00 \%$ & 28 & 1 & 6 \\
\hline Mean & $58.30 \%$ & $54.57 \%$ & $56.98 \%$ & $56.04 \%$ & 25 & 4 & 7 \\
\hline Stddev & $0.8223 \%$ & $7.1607 \%$ & $4.5317 \%$ & $4.6161 \%$ & 10.21 & 3.70 & 3.11 \\
\hline 14 & $60.00 \%$ & $59.33 \%$ & $63.33 \%$ & $66.00 \%$ & 34 & 5 & 9 \\
\hline 15 & $56.95 \%$ & $54.30 \%$ & $60.93 \%$ & $60.93 \%$ & 5 & 10 & 12 \\
\hline 16 & $57.62 \%$ & $57.62 \%$ & $57.62 \%$ & $53.64 \%$ & 41 & 4 & 6 \\
\hline 17 & $57.62 \%$ & $58.28 \%$ & $64.24 \%$ & $64.24 \%$ & 36 & 12 & 21 \\
\hline 18 & $56.67 \%$ & $57.33 \%$ & $64.00 \%$ & $62.00 \%$ & 13 & 10 & 15 \\
\hline Mean & $57.77 \%$ & $57.37 \%$ & $62.02 \%$ & $61.36 \%$ & 24 & 9 & 14 \\
\hline Stddev & $1.1751 \%$ & $1.6815 \%$ & $2.4963 \%$ & $4.2420 \%$ & 15.12 & 3.00 & 5.41 \\
\hline 21 & $56.67 \%$ & $59.33 \%$ & $62.67 \%$ & $74.00 \%$ & 6 & 14 & 20 \\
\hline 22 & $58.28 \%$ & $66.89 \%$ & $61.59 \%$ & $76.82 \%$ & 9 & 5 & 13 \\
\hline 23 & $58.28 \%$ & $62.25 \%$ & $61.59 \%$ & $71.52 \%$ & 7 & 12 & 20 \\
\hline 24 & $60.93 \%$ & $57.62 \%$ & $70.20 \%$ & $74.17 \%$ & 7 & 11 & 17 \\
\hline 25 & $59.33 \%$ & $68.67 \%$ & $72.67 \%$ & $78.67 \%$ & 7 & 14 & 18 \\
\hline Mean & $58.70 \%$ & $62.95 \%$ & $65.74 \%$ & $75.04 \%$ & 8 & 11 & 17 \\
\hline Stddev & $1.4037 \%$ & $4.2475 \%$ & $4.7278 \%$ & $2.4710 \%$ & 0.87 & 3.35 & 2.55 \\
\hline 28 & $58.00 \%$ & $61.33 \%$ & $64.00 \%$ & $72.67 \%$ & 4 & 9 & 19 \\
\hline 29 & $57.62 \%$ & $63.58 \%$ & $59.60 \%$ & $68.21 \%$ & 14 & 8 & 17 \\
\hline 30 & $60.26 \%$ & $72.85 \%$ & $64.90 \%$ & $76.16 \%$ & 10 & 11 & 22 \\
\hline 31 & $59.60 \%$ & $66.23 \%$ & $75.50 \%$ & $76.82 \%$ & 5 & 15 & 18 \\
\hline 32 & $57.33 \%$ & $70.67 \%$ & $71.33 \%$ & $77.33 \%$ & 5 & 12 & 8 \\
\hline Mean & $58.56 \%$ & $66.93 \%$ & $67.07 \%$ & $74.24 \%$ & 9 & 12 & 16 \\
\hline Stddev & $1.1579 \%$ & $4.2907 \%$ & $5.6405 \%$ & $3.4271 \%$ & 3.77 & 2.50 & 5.12 \\
\hline 35 & $56.67 \%$ & $65.33 \%$ & $71.33 \%$ & $73.33 \%$ & 5 & 12 & 20 \\
\hline 36 & $56.95 \%$ & $64.24 \%$ & $68.87 \%$ & $78.15 \%$ & 5 & 7 & 11 \\
\hline
\end{tabular}




\begin{tabular}{|c|c|c|c|c|c|c|c|}
\hline 37 & $54.97 \%$ & $70.86 \%$ & $72.85 \%$ & $81.46 \%$ & 7 & 11 & 12 \\
\hline 38 & $55.63 \%$ & $74.17 \%$ & $68.87 \%$ & $84.11 \%$ & 6 & 7 & 15 \\
\hline 39 & $54.67 \%$ & $72.67 \%$ & $76.67 \%$ & $82.00 \%$ & 11 & 9 & 14 \\
\hline Mean & $55.78 \%$ & $69.45 \%$ & $71.72 \%$ & $79.81 \%$ & 7 & 9 & 13 \\
\hline Stddev & $0.9041 \%$ & $3.9685 \%$ & $2.9013 \%$ & $3.7598 \%$ & 2.28 & 1.66 & 1.58 \\
\hline 42 & $54.00 \%$ & $74.00 \%$ & $76.00 \%$ & $77.33 \%$ & 12 & 11 & 15 \\
\hline 43 & $57.62 \%$ & $72.85 \%$ & $84.77 \%$ & $85.43 \%$ & 6 & 12 & 19 \\
\hline 44 & $58.28 \%$ & $75.50 \%$ & $79.47 \%$ & $76.82 \%$ & 5 & 14 & 18 \\
\hline 45 & $56.95 \%$ & $76.82 \%$ & $82.12 \%$ & $84.11 \%$ & 6 & 9 & 12 \\
\hline 46 & $56.67 \%$ & $80.00 \%$ & $84.00 \%$ & $82.67 \%$ & 8 & 15 & 17 \\
\hline Mean & $56.70 \%$ & $75.83 \%$ & $81.27 \%$ & $81.27 \%$ & 6 & 13 & 17 \\
\hline Stddev & $1.4618 \%$ & $2.4789 \%$ & $3.2070 \%$ & $3.5381 \%$ & 1.09 & 2.29 & 2.69 \\
\hline 49 & $58.00 \%$ & $78.67 \%$ & $82.67 \%$ & $86.00 \%$ & 6 & 12 & 15 \\
\hline 50 & $58.94 \%$ & $62.91 \%$ & $81.46 \%$ & $78.81 \%$ & 4 & 15 & 19 \\
\hline 51 & $57.62 \%$ & $81.46 \%$ & $87.42 \%$ & $80.13 \%$ & 5 & 14 & 19 \\
\hline 52 & $54.67 \%$ & $74.00 \%$ & $82.00 \%$ & $83.33 \%$ & 6 & 10 & 16 \\
\hline 53 & $54.67 \%$ & $74.67 \%$ & $77.33 \%$ & $84.00 \%$ & 8 & 13 & 18 \\
\hline Mean & $56.78 \%$ & $74.34 \%$ & $82.17 \%$ & $82.45 \%$ & 6 & 13 & 18 \\
\hline Stddev & $1.7769 \%$ & $6.3269 \%$ & $3.2159 \%$ & $2.6238 \%$ & 1.48 & 1.87 & 1.22 \\
\hline 56 & $51.66 \%$ & $68.87 \%$ & $75.50 \%$ & $75.50 \%$ & 5 & 15 & 16 \\
\hline 57 & $55.63 \%$ & $74.17 \%$ & $77.48 \%$ & $82.12 \%$ & 5 & 15 & 16 \\
\hline 58 & $54.97 \%$ & $78.15 \%$ & $72.85 \%$ & $84.11 \%$ & 14 & 12 & 19 \\
\hline 59 & $54.00 \%$ & $70.00 \%$ & $75.33 \%$ & $80.00 \%$ & 4 & 8 & 16 \\
\hline 60 & $50.67 \%$ & $80.67 \%$ & $67.33 \%$ & $78.00 \%$ & 6 & 8 & 8 \\
\hline Mean & $53.38 \%$ & $74.37 \%$ & $73.70 \%$ & $79.94 \%$ & 7 & 11 & 15 \\
\hline Stddev & $1.9129 \%$ & $4.5441 \%$ & $3.5063 \%$ & $3.0204 \%$ & 3.96 & 2.95 & 4.09 \\
\hline
\end{tabular}




\section{Appendix B Maltab Codes}

SVM based prediction with AHCFS as a feature selection method

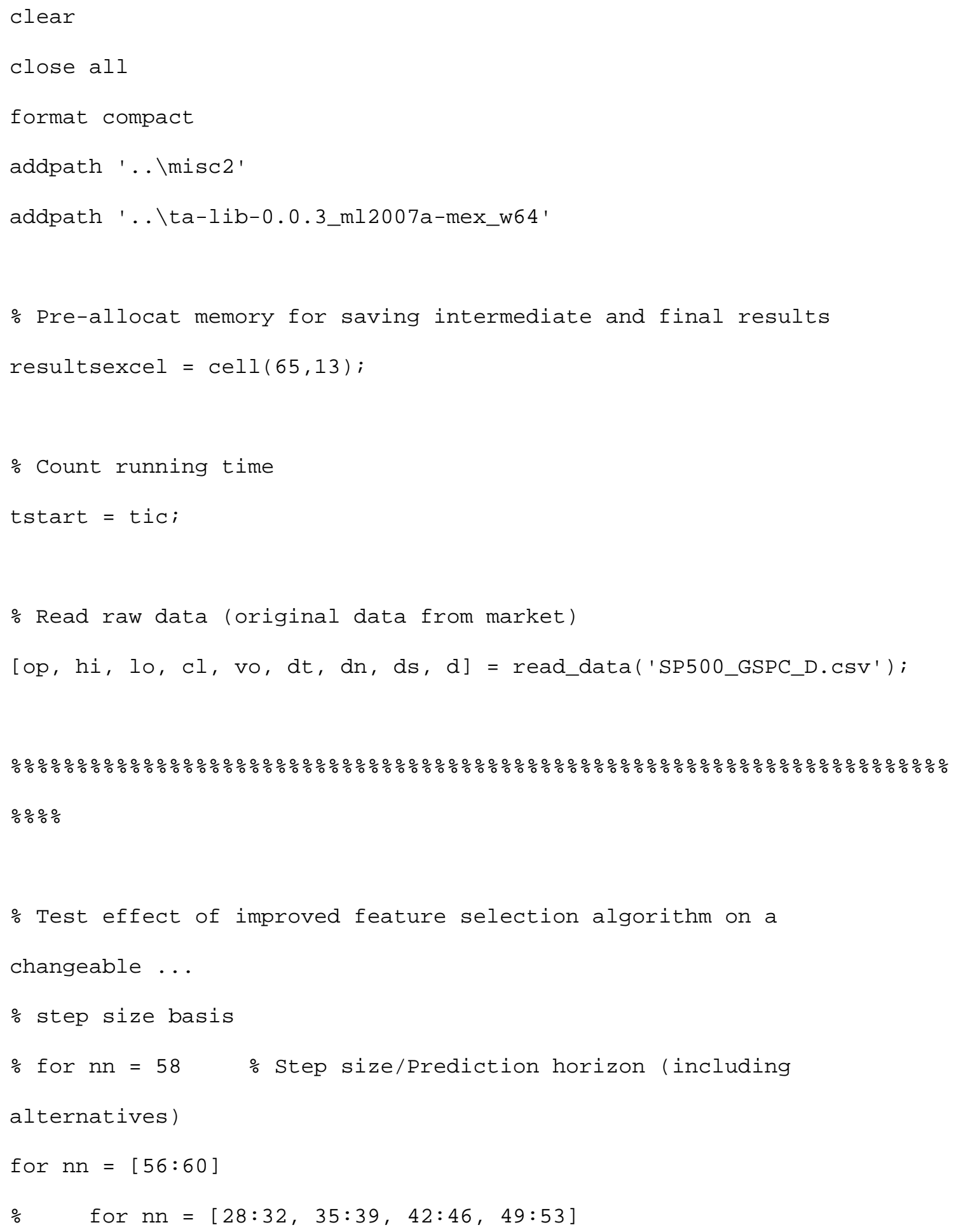




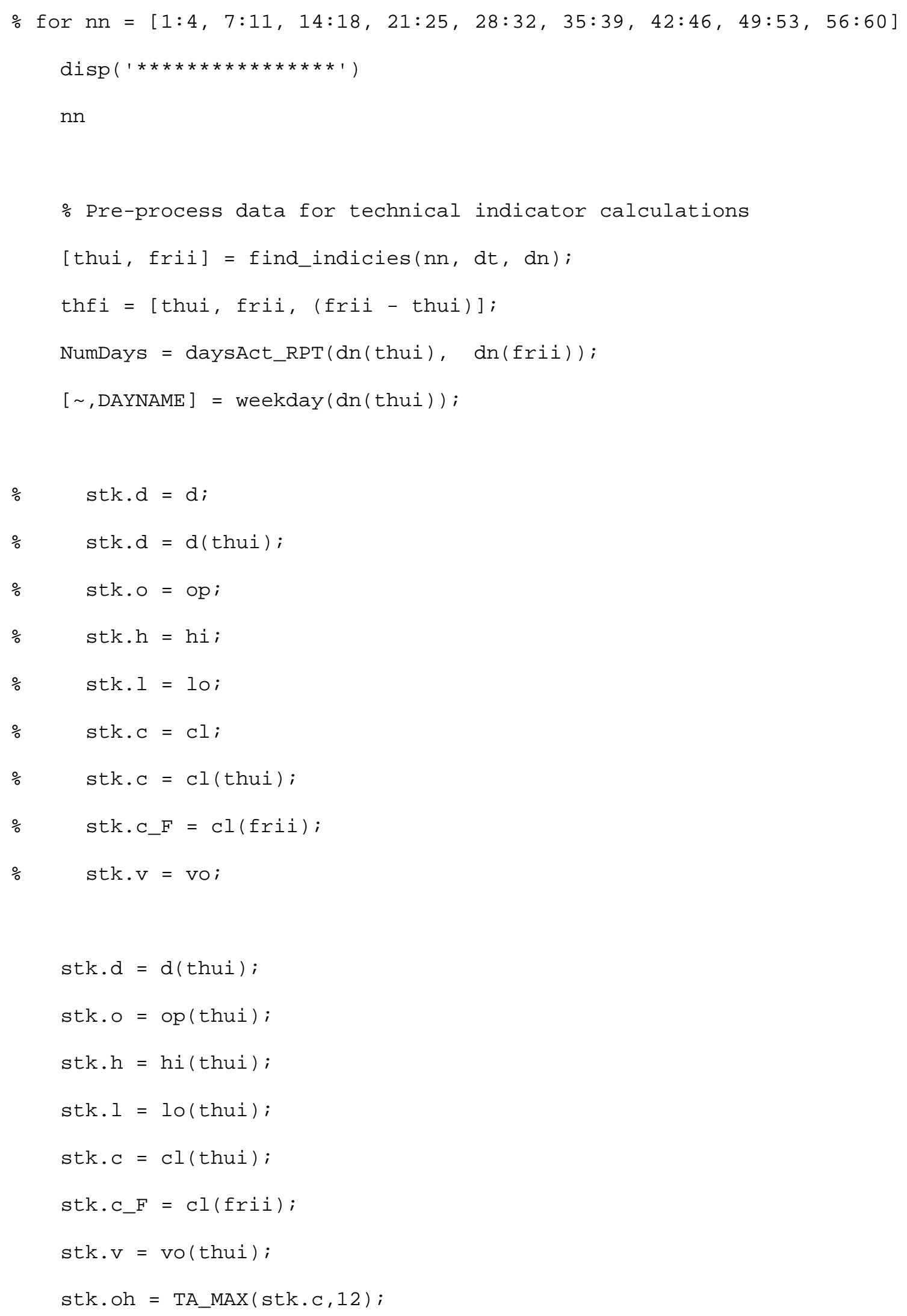


stk.ol = TA_MIN(stk.c, 12);

\%\%\%\%\%\%\%\%\%\%\%\%\%\%\%\%\%\%\%\%\%\%\%\%\%\%\%\%\%\%\%\%\%\%\%\%\%\%\%\%\%\%\%\%\%\%\%\%\%\%\%\%\%\%\%\%\%\%\% $\% \% \%$

\% Calculate technical indicators, further create feature set and $\%$ training target set as SVM inputs

features_store $=$ technical_indicators $($ stk $)$;

features_store $($ isnan $($ features_store $))=0$;

features = features_store;

features_copy = features;

CorrectTargets $=$ stk. $\mathrm{C}_{-} \mathrm{F}>=\mathrm{stk} \cdot \mathrm{C}$;

\%\%\%\%\%\%\%\%\%\%\%\%\%\%\%\%\%\%\%\%\%\%\%\%\%\%\%\%\%\%\%\%\%\%\%\%\%\%\%\%\%\%\%\%\%\%\%\%\%\%\%\%\%\%\%\%\%\% $\% \%$

\% Create time label to partition data for training and testing aims

T1 $=$ find (stk.d >= 20020101, 1); \% T1 - T2: IS' for filter method

$\mathrm{T} 2=$ find $($ stk.d $>=20040101,1) ; \% \mathrm{~T} 2$ - T3: 00S' for fitler method

T3 = find(stk.d >= 20090101, 1); \% T1 - T3: IS for SVM training

T4 $=$ find $($ stk.d $>=20120101,1) ; \%$ T3 - T4: 00S for SVM testing

IS_Set_FS $=$ features $(\mathrm{T} 1: \mathrm{T} 2-1,:)$;

IS_CorrectTargets_FS $=$ CorrectTargets $(\mathrm{T} 1: \mathrm{T} 2-1) ;$

00S_Set_FS $=$ features $(T 2: T 3-1,:) ;$

00S_CorrectTargets_FS $=$ CorrectTargets $($ T2:T3-1); 


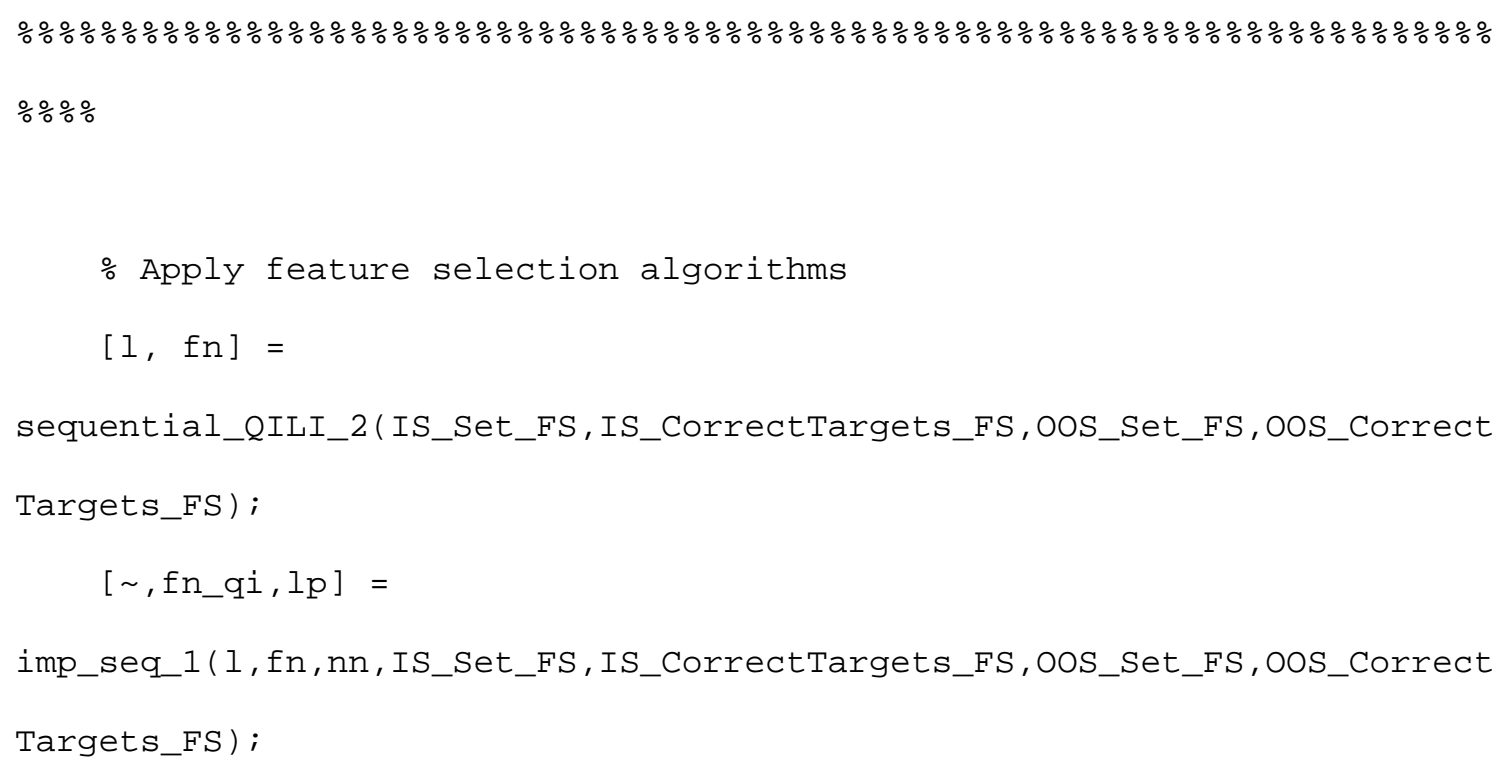


resultsexcel = store_results_imp_seq $\left(\right.$ resultsexcel, nn,fn_qi, $\left.1 \_q i\right)$;

end

telapsed $=$ toc $($ tstart $)$

telapsed_min $=$ telapsed $/ 60$

telapsed_hr $=$ telapsed_min/60

resultsexcel = write_to_excel $($ resultsexcel $)$; 
SVM based prediction with ERFS as a feature selection method

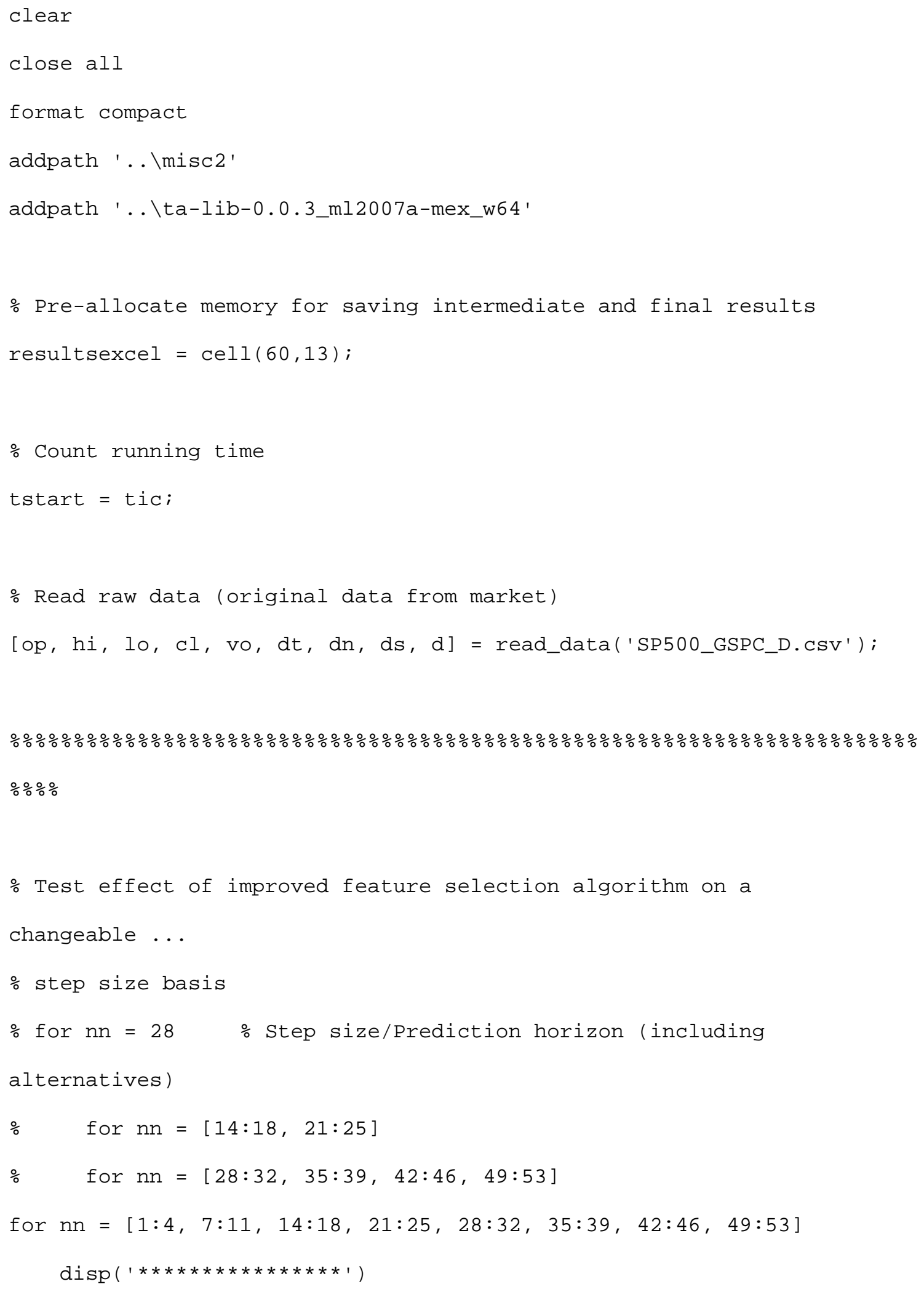


nn

\% Pre-process data for technical indicator calculations

[thui, frii] = find_indicies $(\mathrm{nn}, \mathrm{dt}, \mathrm{dn})$;

thfi $=[$ thui, frii, (frii - thui) $]$;

NumDays $=$ daysAct_RPT $(\operatorname{dn}($ thui $), \quad \operatorname{dn}($ frii $))$;

$[\sim$, DAYNAME $]=$ weekday $(\operatorname{dn}($ thui $))$;

$\% \quad$ stk.d = d;

$\% \quad$ stk.d = d(thui);

\% stk.o = op;

$\% \quad$ stk.h = hi;

\% stk.l = 10;

$\% \quad$ stk.c $=\mathrm{cl}$;

$\% \quad$ stk.c $=c l($ thui $) ;$

$\% \quad$ stk.C_F $=\mathrm{cl}($ frii $)$;

$\%$ stk.v = vo;

stk.d = d(thui);

stk.o = op(thui);

stk.h = hi(thui);

stk.1 = lo(thui);

stk.c = cl(thui);

stk.c_F = cl(frii);

stk.v = vo(thui);

stk.oh = TA_MAX(stk.c,12);

stk.ol = TA_MIN (stk.c,12); 
\% Calculate technical indicators, further create feature set and $\%$ training target set as SVM inputs

features_store = technical_indicators $($ stk $)$;

features_store $($ isnan $($ features_store $))=0$;

features = features_store;

features_copy $=$ features;

CorrectTargets $=$ stk. $\mathrm{C}_{-} \mathrm{F}>=\mathrm{stk} \cdot \mathrm{C} ;$

\%\%\%\%\%\%\%\%\%\%\%\%\%\%\%\%\%\%\%\%\%\%\%\%\%\%\%\%\%\%\%\%\%\%\%\%\%\%\%\%\%\%\%\%\%\%\%\%\%\%\%\%\%\%\%\%\%\%\%\%\%\%\%\% $\% \% \%$

\% Create time label to partition data for training and testing aims

$\mathrm{T} 1=$ find $(\mathrm{stk} \cdot \mathrm{d}>=20030101,1) ; \% \mathrm{~T} 1$ - T2: IS' for filter method

T2 = find (stk.d >= 20100101, 1); \% T2 - T3: 00S' for filter method

T3 $=$ find $($ stk.d $>=20130101,1) ; \%$ T1 - T3: IS for SVM training

T4 = find (stk.d >= 20170101, 1); \% T3 - T4: 00S for SVM testing

IS_Set_FS $=$ features $(\mathrm{T} 1: \mathrm{T} 2-1,:)$;

IS_CorrectTargets_FS = CorrectTargets $(\mathrm{T} 1: \mathrm{T} 2-1)$;

00S_Set_FS = features $(\mathrm{T} 2: \mathrm{T} 3-1,:)$;

00S_CorrectTargets_FS = CorrectTargets $($ T2:T3-1);

IS_Set_SFS $=$ features $(\mathrm{T} 1: \mathrm{T} 3-1,:)$;

00S_set $=$ features $(\mathrm{T} 3: \mathrm{T} 4,:)$;

IS_CorrectTargets_SFS = CorrectTargets $(\mathrm{T} 1: \mathrm{T} 3-1)$; 
00S_CorrectTargets $=$ CorrectTargets $($ T3:T4 $)$;

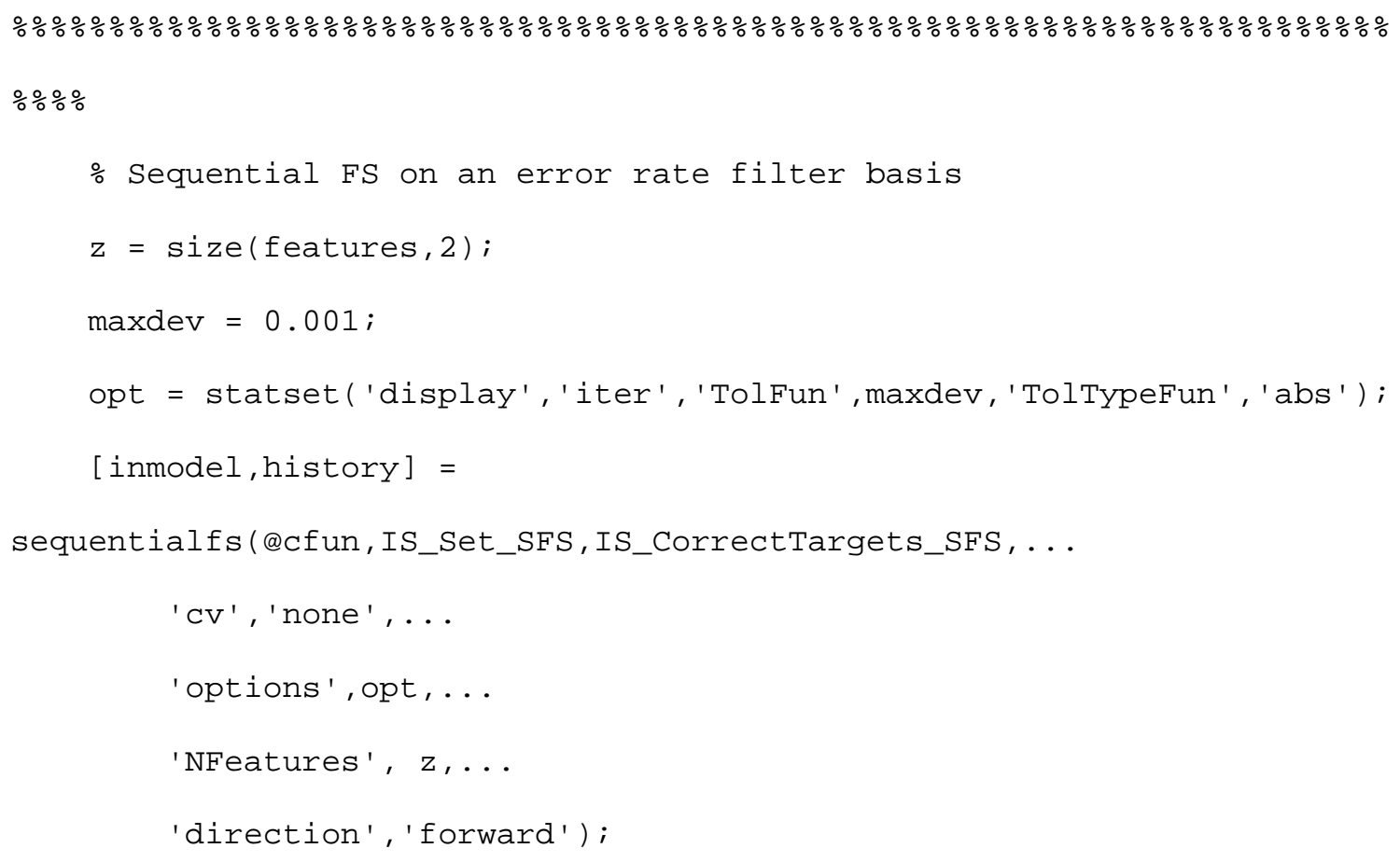

$\%$ Test prediction accuracies of all feature combination on testing data

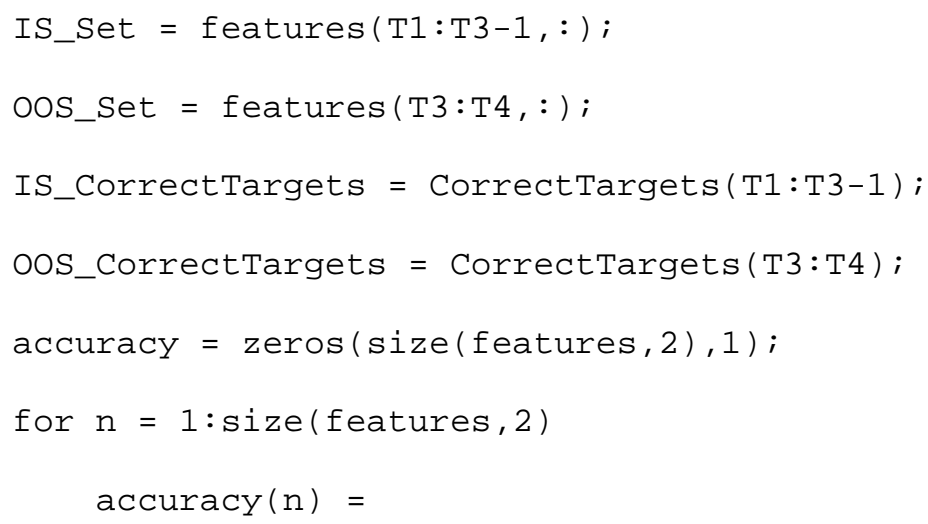




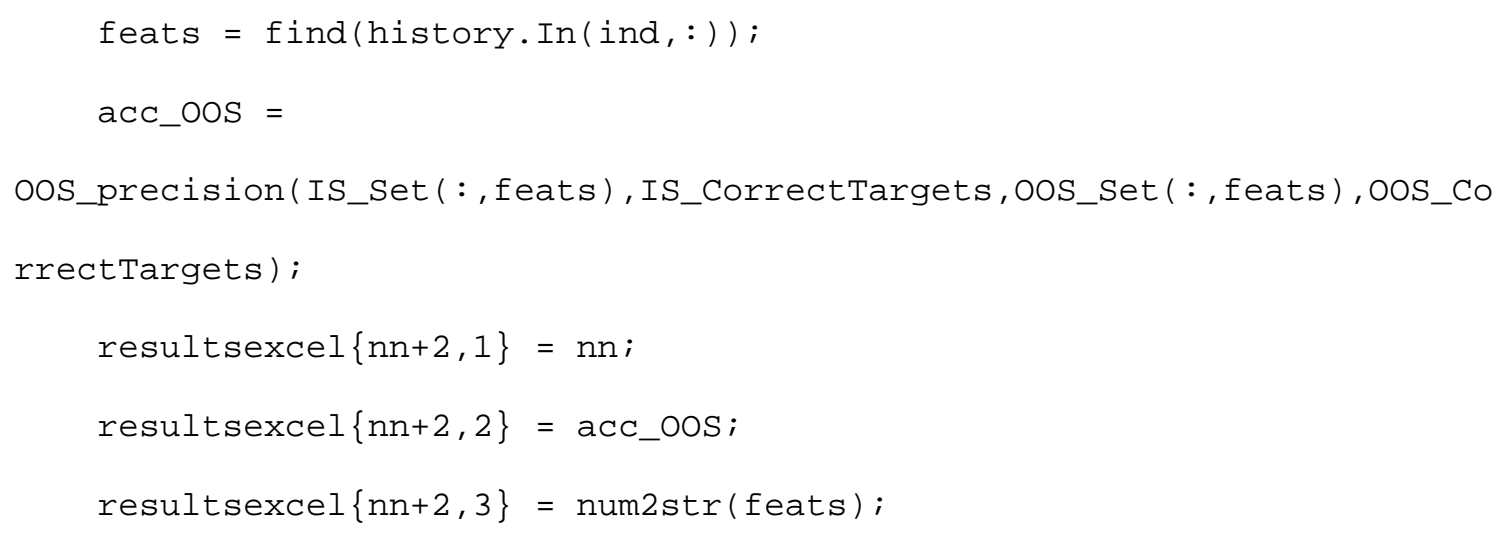


SVM based prediction without feature selection

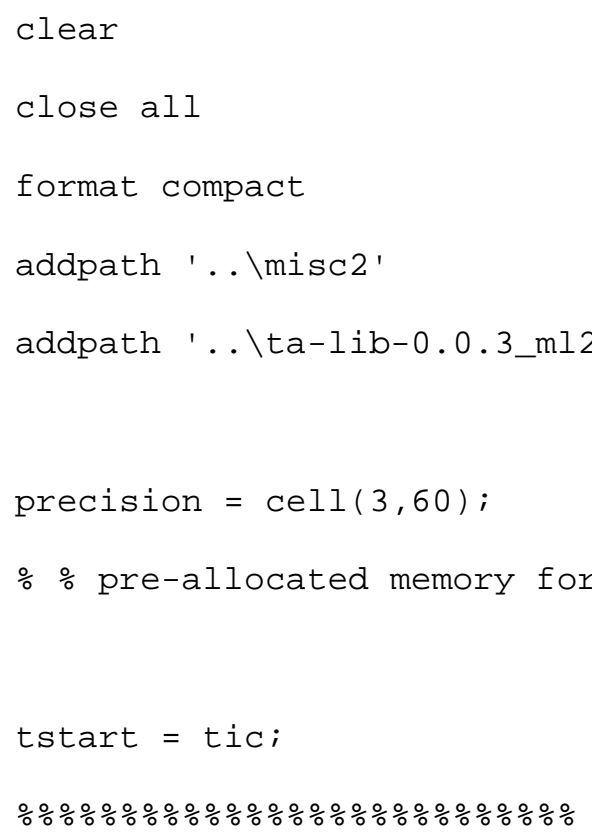




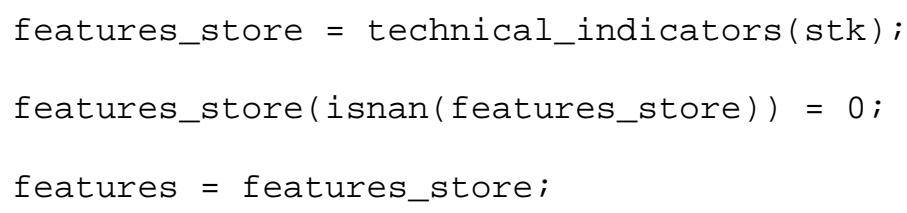

end

telapsed $=$ toc $($ tstart $)$ 
telapsed_min $=$ telapsed $/ 60$
telapsed_hr $=$ telapsed_min $/ 60$

resultsexcel = write_to_excel_withoutFS $($ resultsexcel $)$; 


\section{Sub Functions}

cfun.m

function errRate $=\operatorname{cfun}(X, Y)$

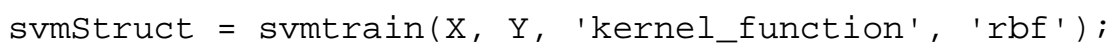

$\mathrm{YY}=\operatorname{svmclassify}($ svmStruct, $\mathrm{X})$;

$\operatorname{errRate}=\operatorname{sum}(\mathrm{YY} \sim=\mathrm{Y}) /$ length $(\mathrm{Y}) ; \%$ mis-classification rate

daysAct_RPT.m

function NumDays $=\operatorname{daysAct}($ StartDate, EndDate $)$

\%daysact Actual number of days between dates

$\%$ Given two dates in serial date numbers or date strings, calculate the

$\%$ actual number of days between them

$\%$

$\%$ NumDays $=\operatorname{daysact}($ StartDate, EndDate $)$

$\%$

\% Inputs:

\% StartDate (required) - The starting date in serial date number or date

$\%$ string format

\% EndDate (optional) - The ending date in serial date number or date

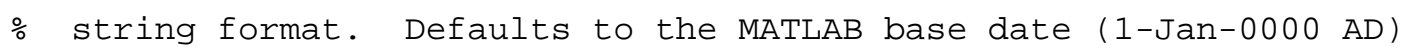

\% Author(s): B. Blunk 02/11/05 bblunk@unlnotes.unl.edu 


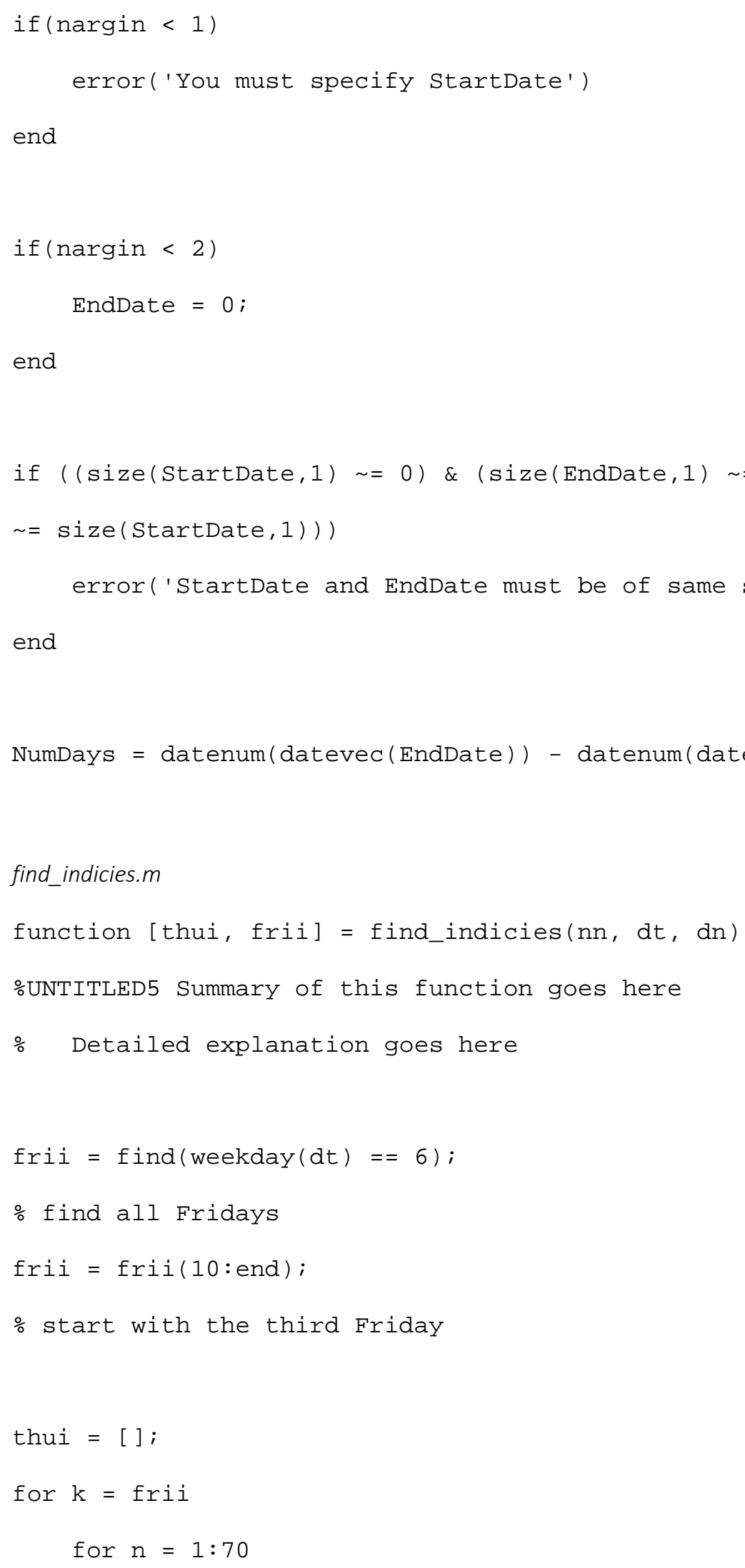




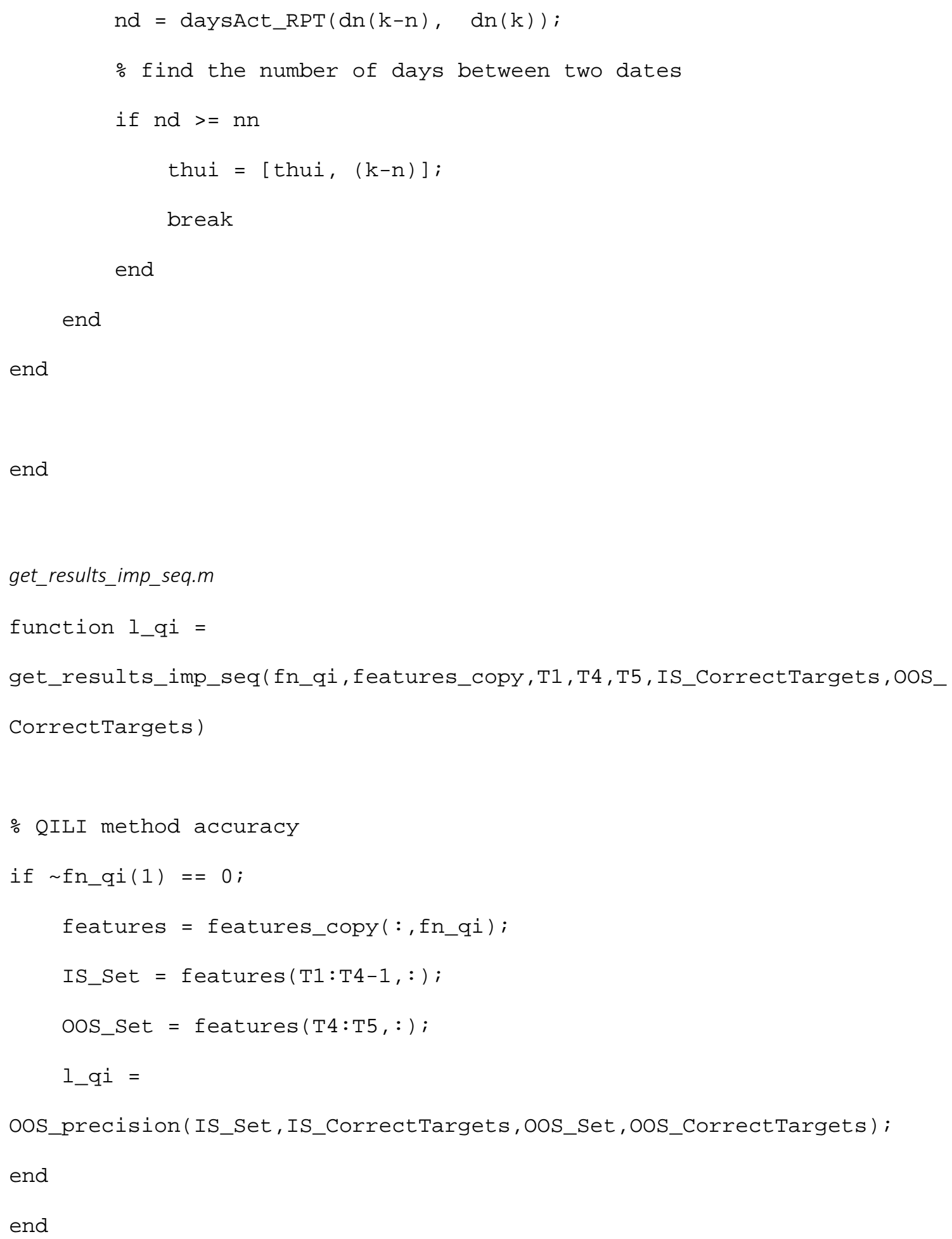




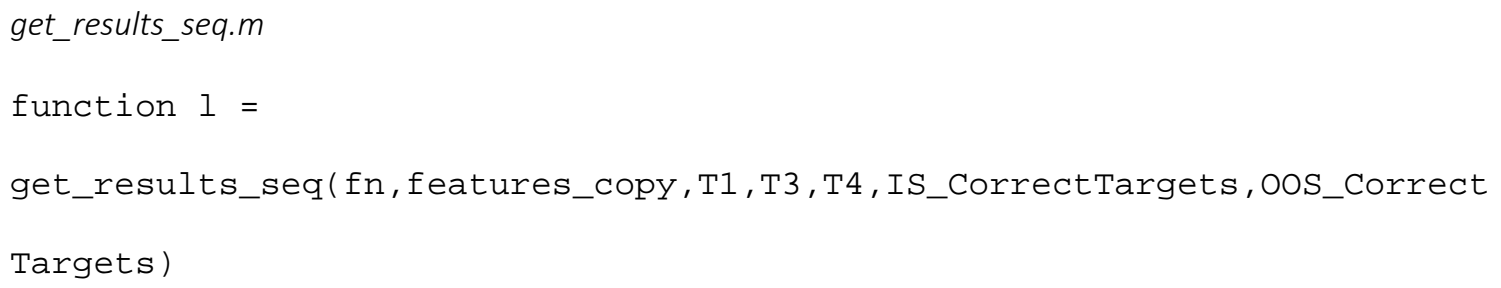




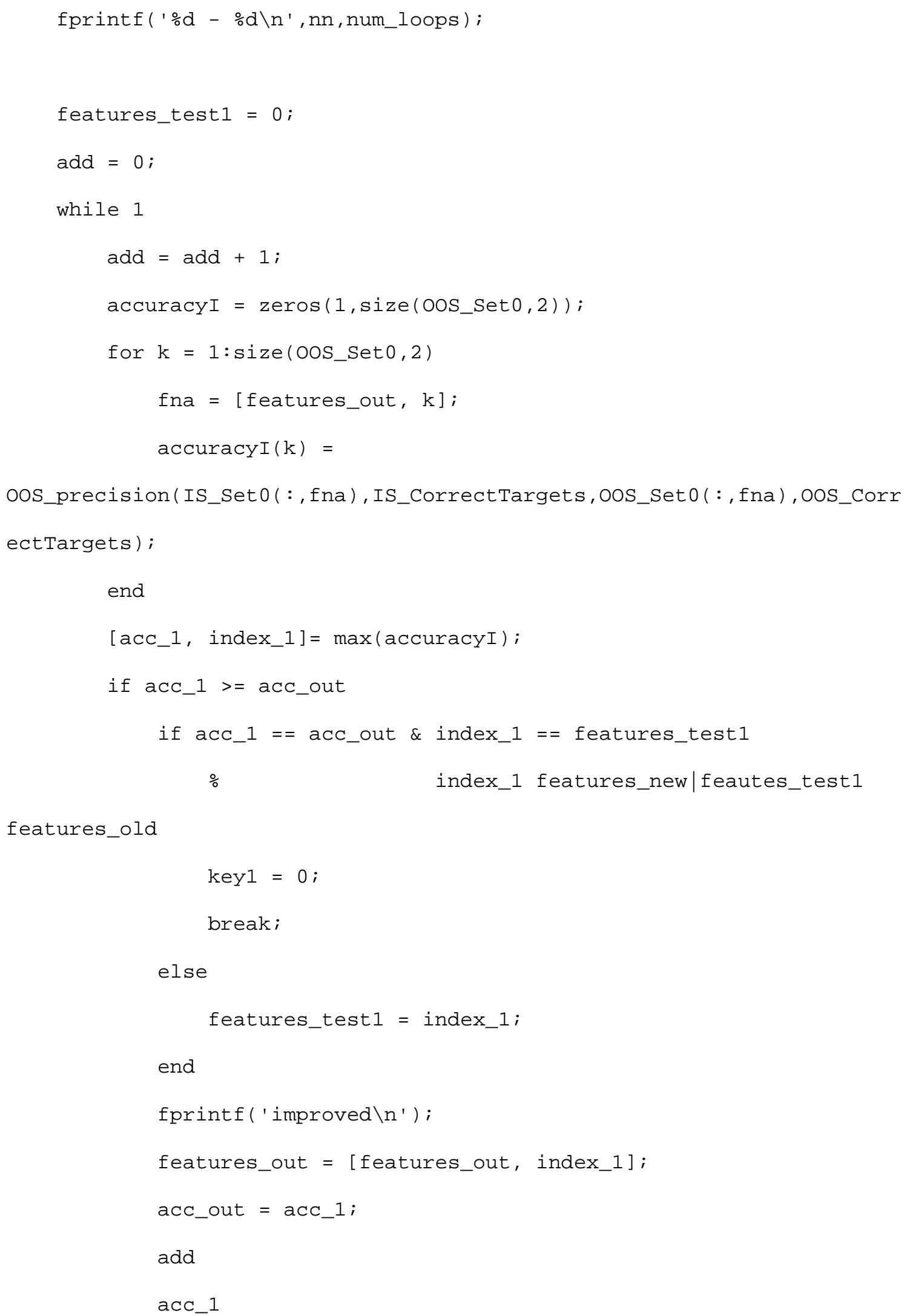




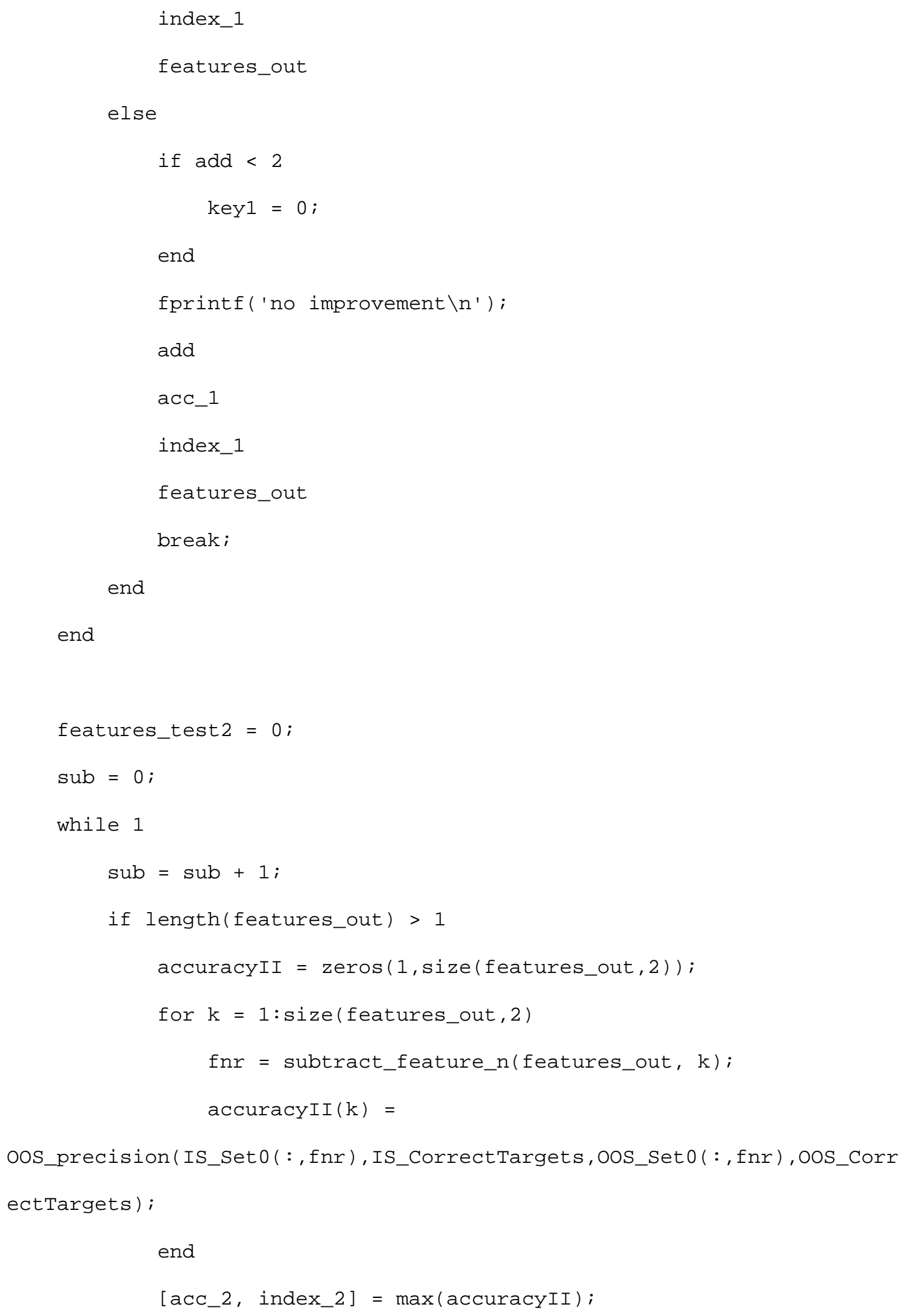




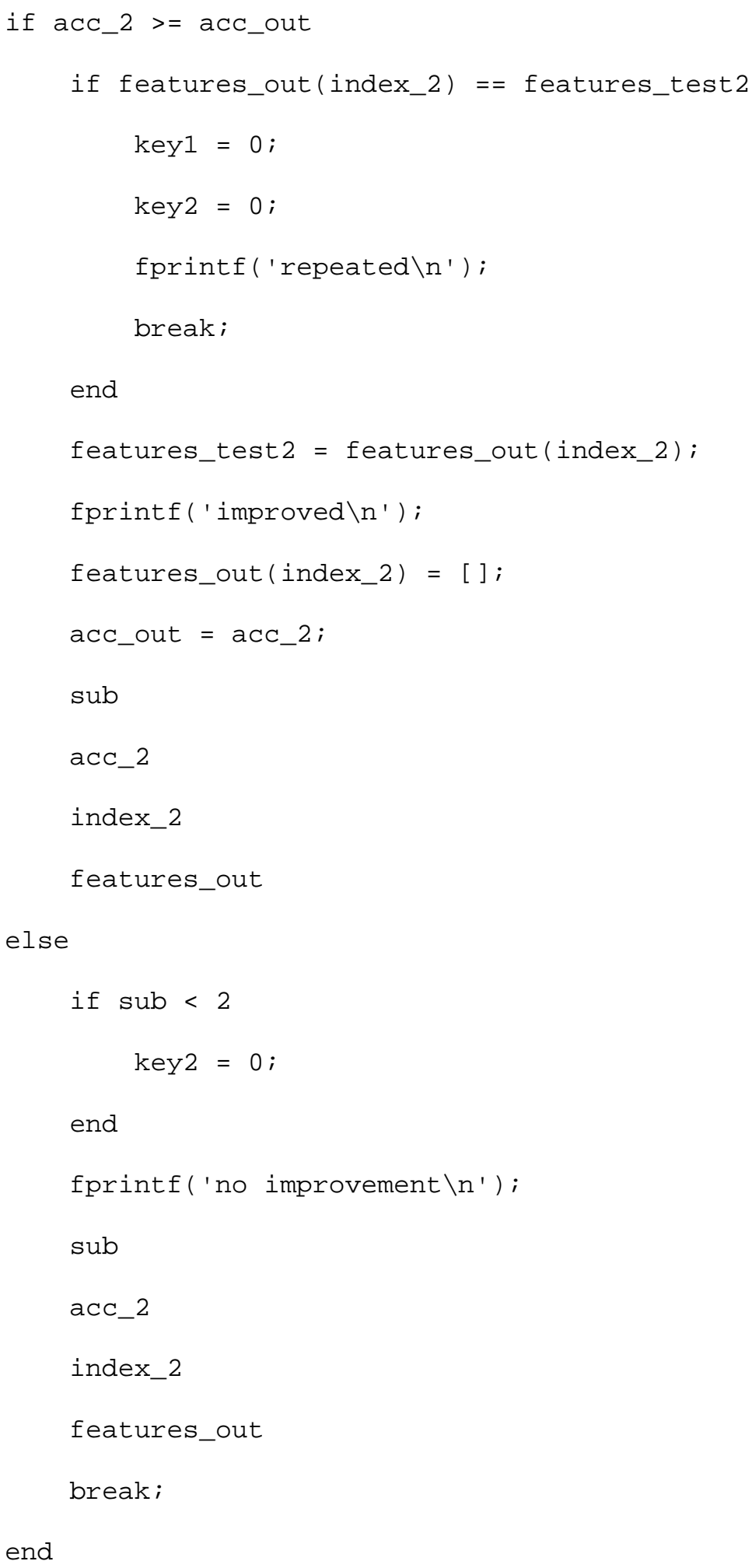


key2 = 0 ;

fprintf('only one selected feature $\backslash n '$ ');

break;

end

end

if acc_out == acc_test1

acc_test2 $=$ acc_test $2+1$;

end

acc_test1 = acc_out;

if $($ key1 || key2 $)==0||$ acc_test2 $==1$

key $=0$;

end

num_loops $=$ num_loops +1 ;

end

if $x>1$

if acc_out <= acc_previous

break;

end

end

acc_previous = acc_out ;

end

end

function features_1 = subtract_feature_n(features, n) 


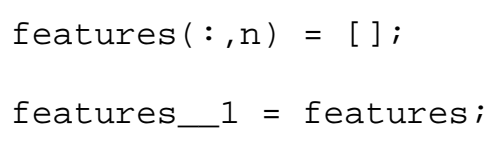

end

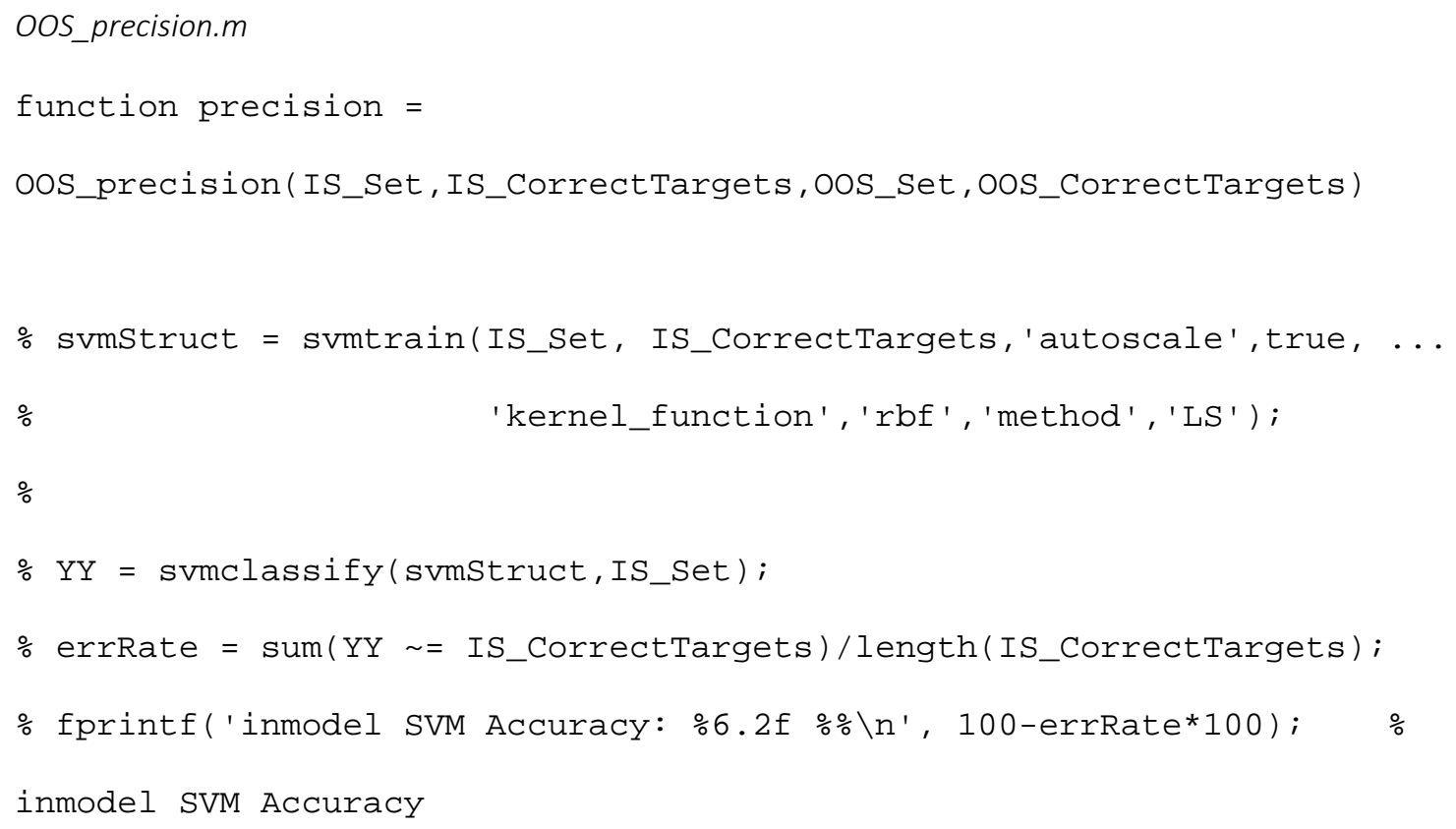




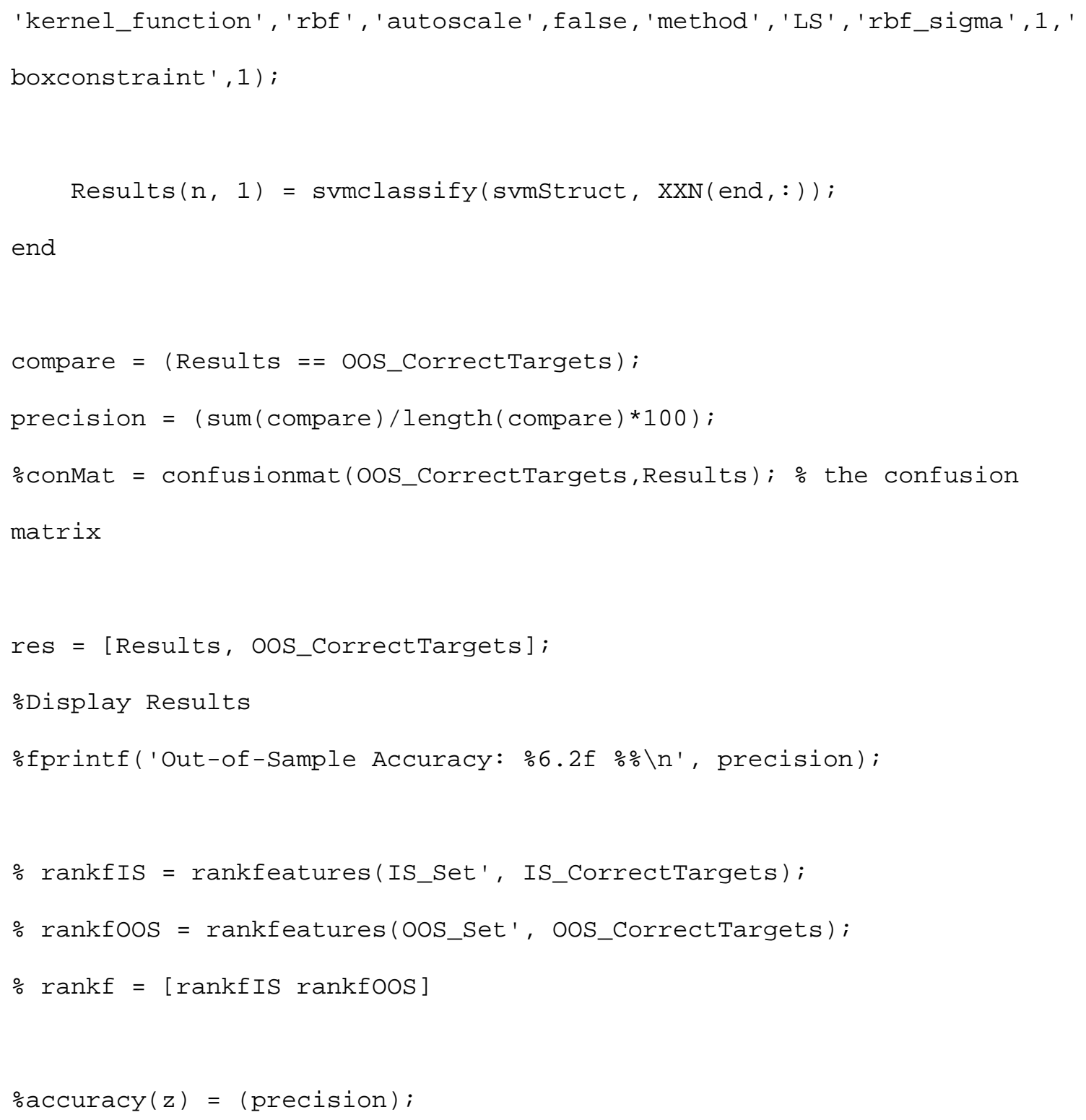




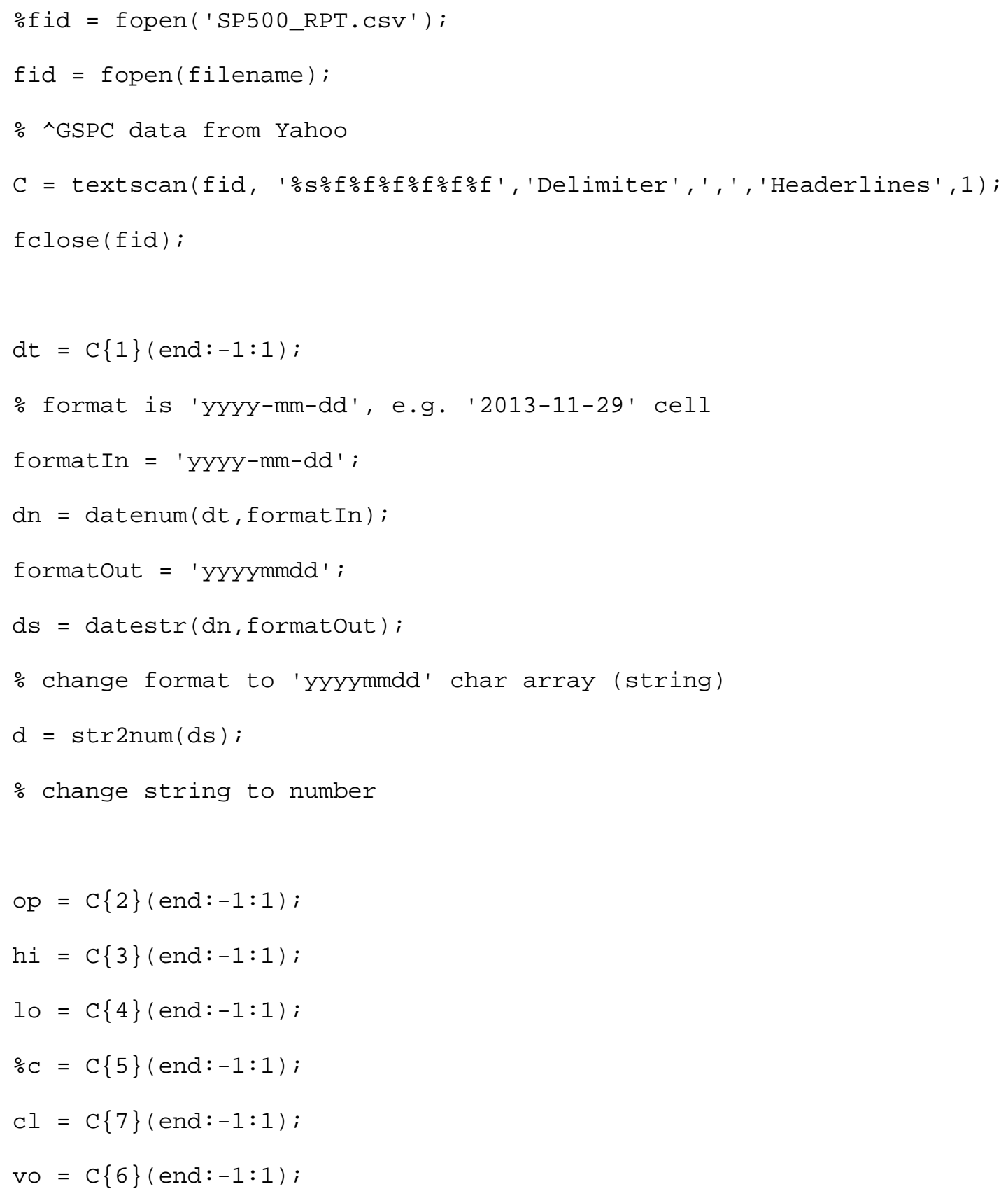

end 
sequential_QILI_2.m

function [acc_seq, feats] =

sequential_QILI(IS_Set $\odot$, IS_CorrectTargets, 00S_Set $\odot, 00 S \_C o r r e c t T a r g e t s$ )

$z=15$

[accuracy, his $]=$

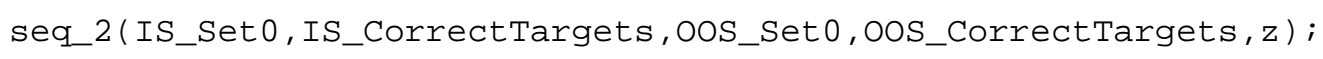

[acc_seq, acc_seq_i] $=\max ($ accuracy $)$;

\% Check intermediate results

fprintf('First \%d In-Sample Accuracies of feature combinations: $\backslash n ', z)$; accuracy

feats $=$ find $($ his $\cdot a($ acc_seq_i, $:))$;

\%Check intermediate results

fprintf('Best combination and In-Sample Accuracy: \n');

feats

acc_seq

end

store_results_imp.m

function [acc_seq, feats] =

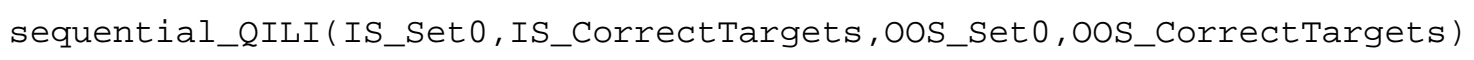

$z=15$;

[accuracy, his $]=$

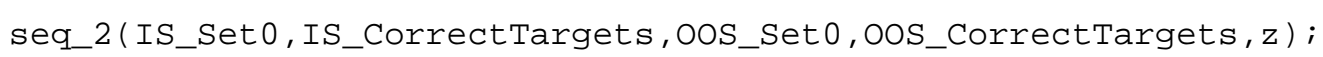




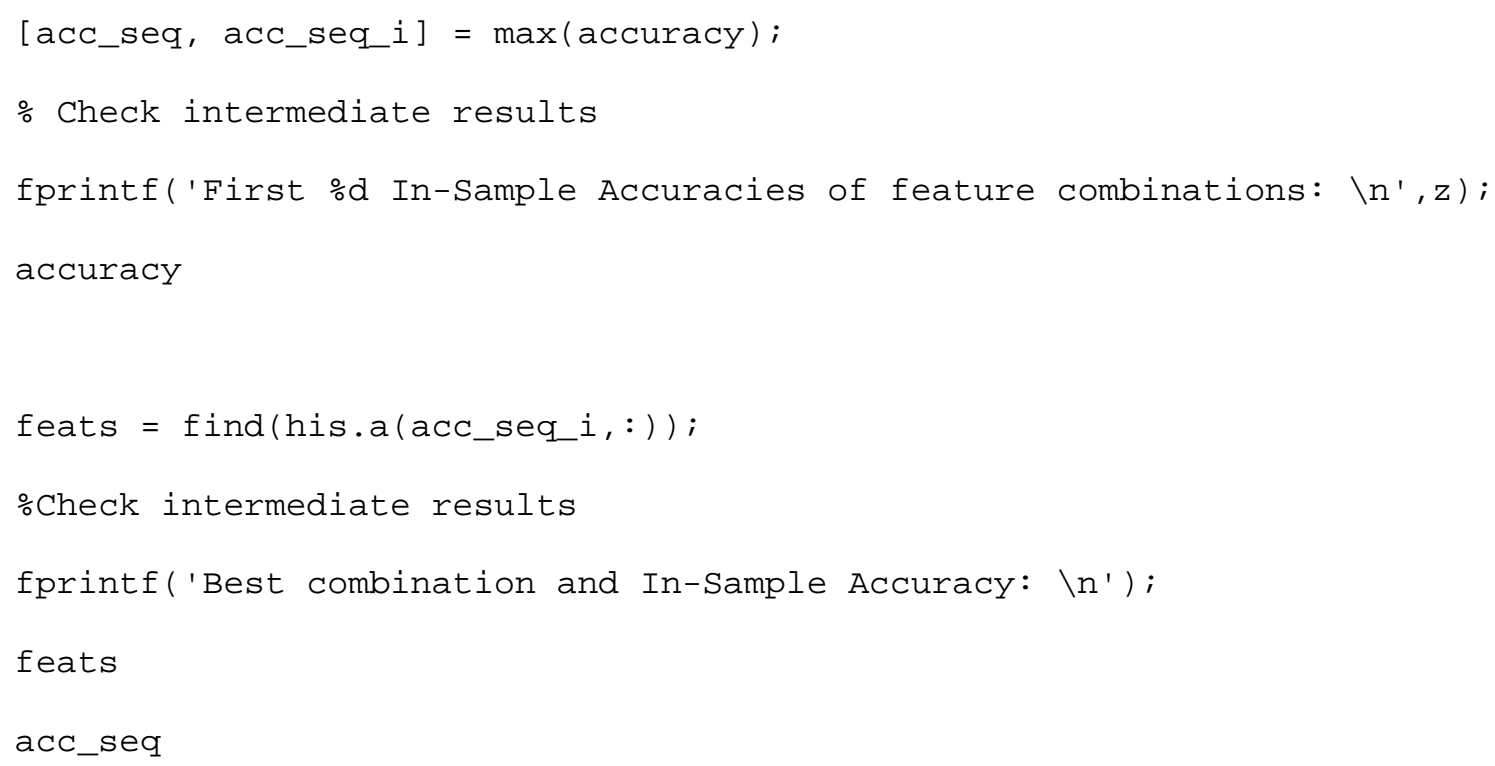


resultsexcel $\{n n+2,10\}=\operatorname{sum}(\operatorname{diff}($ targets $) \sim=0) /$ len; $\%$ different from previous

$\%$ resultsexcel $\{n n+2,4\}=m ; \% \operatorname{num} 2 \operatorname{str}(m) ; \% m ;$

end

store_results_seq.m

function [ resultsexcel ] = store_results_seq(resultsexcel, nn, l, fn)

resultsexcel $\{\mathrm{nn}+2,2\}=1$;

resultsexcel $\{n n+2,3\}=\operatorname{num} 2 \operatorname{str}(f n) ;$

end

technical_indicators.m

function [ indicators ] = technical_indicators(stk)

\%UNTITLED Summary of this function goes here

\% Detailed explanation goes here

\% Calculate Technicals

I1 = TA_RSI (stk.c, 14);

[bBandsHigh, bBandsMid, bBandsLow $]=$ TA_BBANDS(stk.c, 9, 2, 2);

I2 = (stk.c - bBandsHigh) $\cdot /$ bBandsHigh;

I3 = (stk.c - bBandsLow) $\cdot /$ bBandsLow; 
[stochK, stochD] = TA_STOCHF(stk.oh,stk.ol,stk.c, 14, 3);\% 14,3

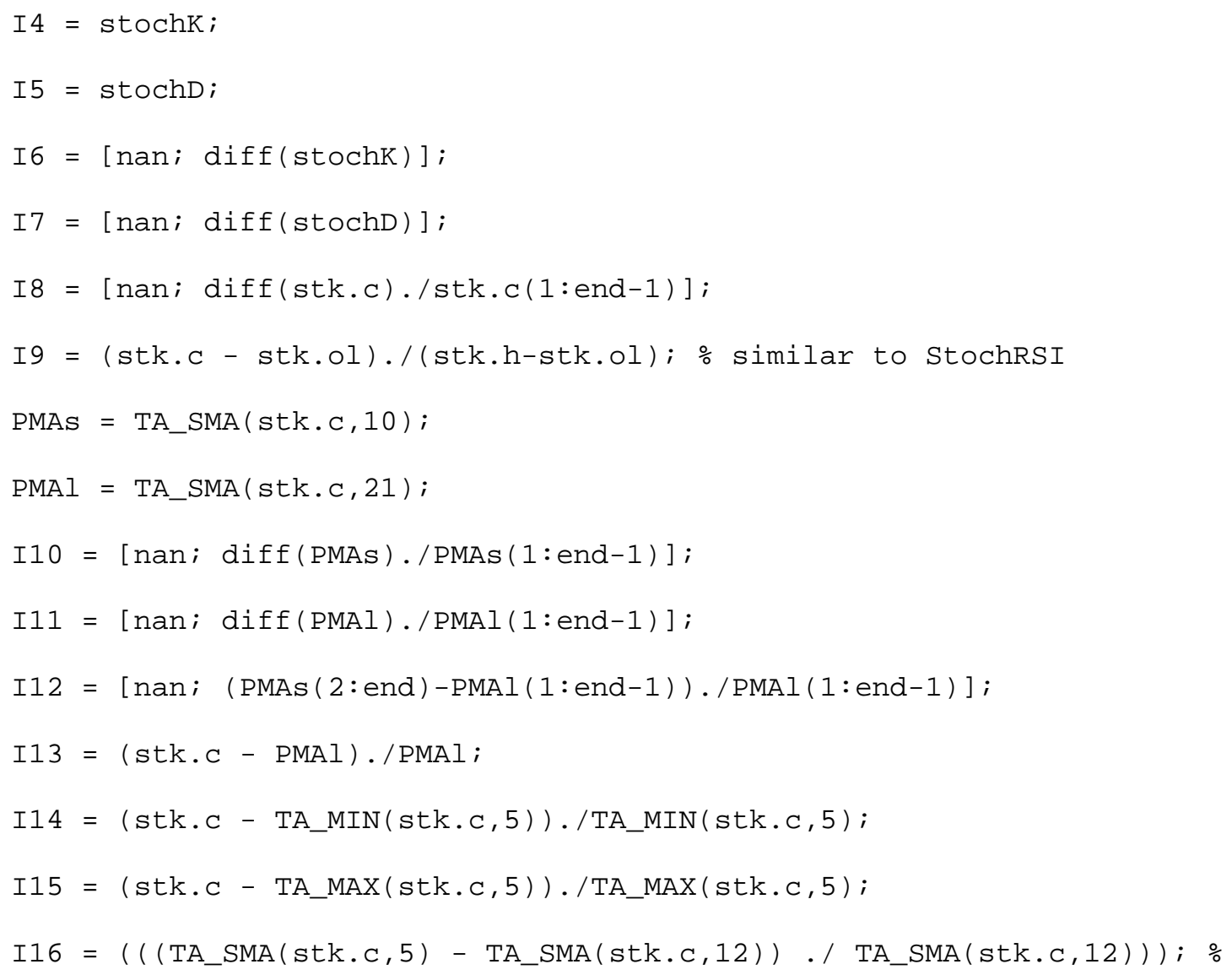




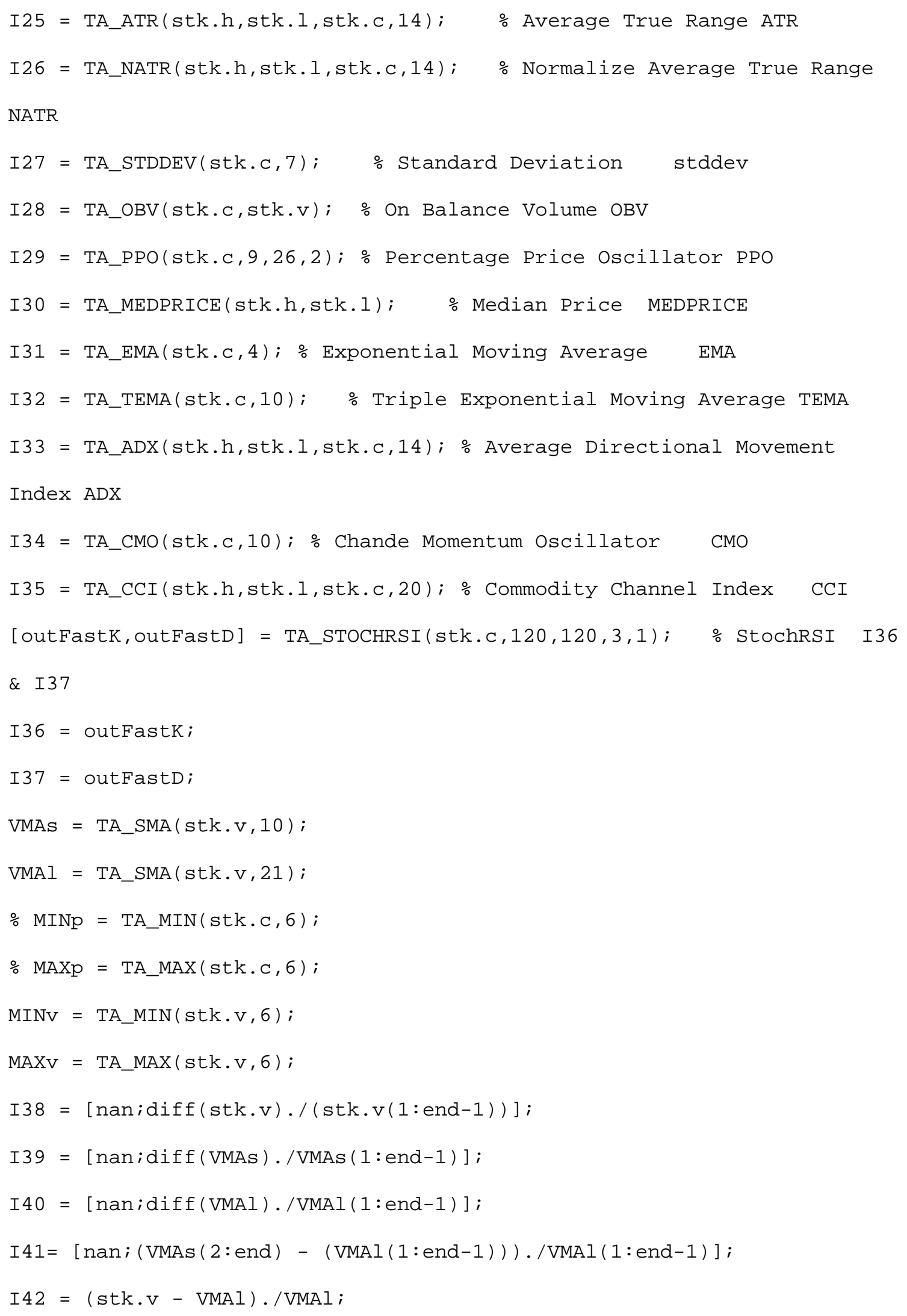




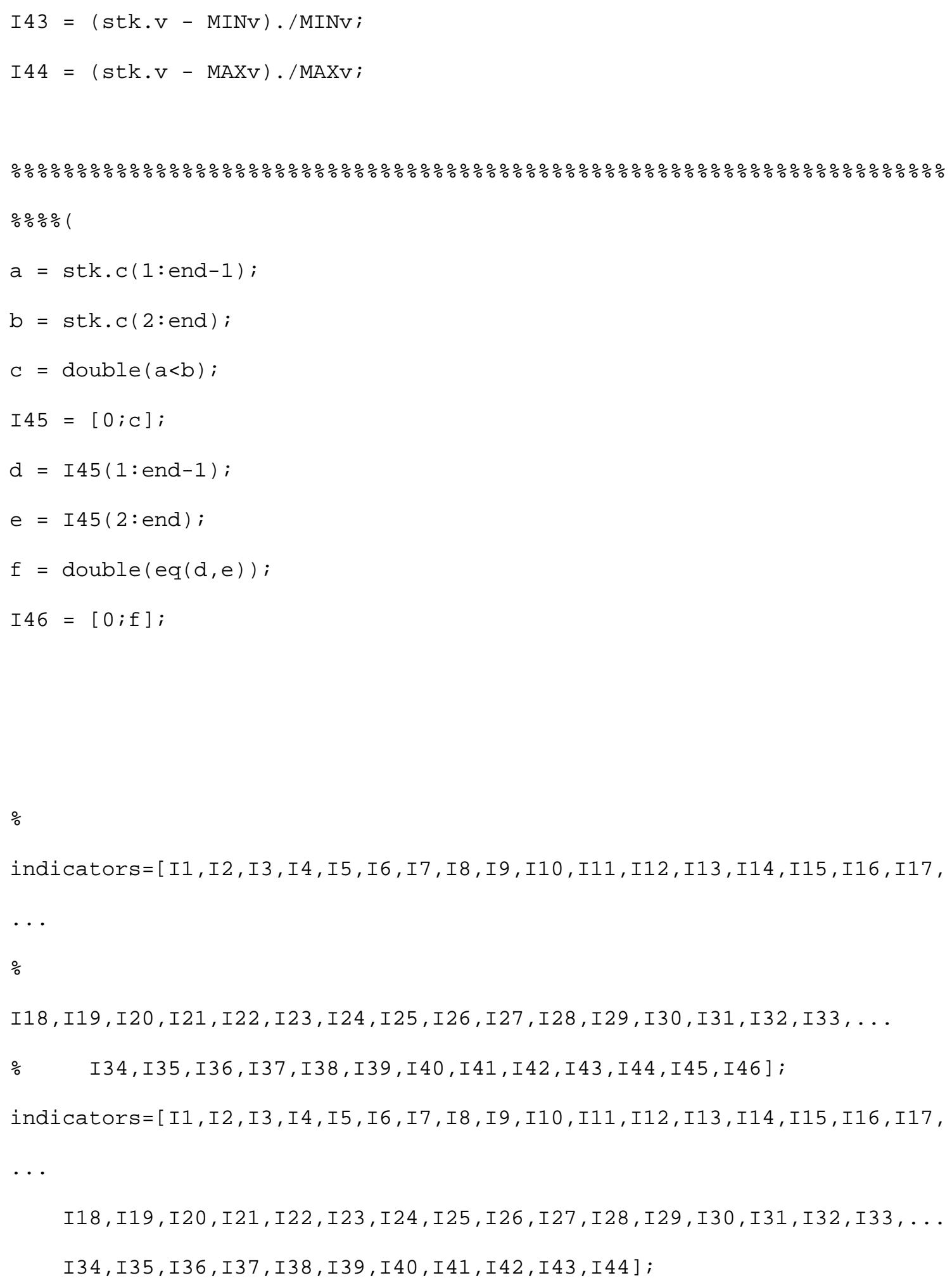


end

write_to_excel.m

function resultsexcel $=$ write_to_excel $($ resultsexcel $)$

$\% \%$ add some titles for excel

resultsexcel $\{1,1\}=$ 'number of day';

resultsexcel $\{1,2\}=$ 'sequentialfs results only';

resultsexcel $\{1,3\}='$ ';

resultsexcel $\{1,4\}=$ 'sequentialfs results plus extra procedure';

resultsexcel $\{1,5\}='$ ';

resultsexcel $\{1,6\}=$ 'number of loop';

resultsexcel $\{2,1\}='$ ';

resultsexcel $\{2,2\}=$ 'accuracy\%';

resultsexcel $\{2,3\}=$ 'selected features';

resultsexcel $\{2,4\}=$ 'accuracy\%';

resultsexcel $\{2,5\}=$ 'selected features';

resultsexcel $\{2,6\}='$ ';

xlswrite('results', resultsexcel); \% create excel of results

end 


\section{Appendix C Supplemental Files}

\begin{tabular}{|l|l|l|l|}
\hline$\#$ & Name & File Type & Size \\
\hline 1 & $2007-2017$. ods & OpenDocument Spreadsheet & 446 KB \\
\hline 2 & $2000-2010$. ods & OpenDocument Spreadsheet & 417 KB \\
\hline 3 & $1992-2002$. ods & OpenDocument Spreadsheet & 401 KB \\
\hline 4 & $1960-1970$. ods & OpenDocument Spreadsheet & 387 KB \\
\hline
\end{tabular}

The above four spreadsheets are reorganized unrefined data from the MATLAB codes in Appendix $B$. The codes which give the above data are all the same except the time frame variables.

\section{Software requirements:}

Any OpenDocument supported software, including Microsoft Excel, Calligra Sheets, EditGrid, Google Docs and many more, can access above files. Refer to this link to find out more OpenDocument supported software:

https://en.wikipedia.org/wiki/OpenDocument software\#Spreadsheet documents (.ods)

\section{Hardware requirements:}

No special requirements for hardware. 